Near-inertial and Thermal Upper Ocean Response

to Atmospheric Forcing in the North Atlantic

Ocean

\author{
by \\ Katherine E. Silverthorne \\ B.S., Southwestern University (2003) \\ Submitted in partial fulfillment of the \\ requirements for the degree of \\ Doctor of Philosophy in Physical Oceanography \\ at the \\ MASSACHUSETTS INSTITUTE OF TECHNOLOGY \\ and the \\ WOODS HOLE OCEANOGRAPHIC INSTITUTION \\ June 2010 \\ (c) Katherine E. Silverthorne, 2010
}

The author hereby grants to MIT and to WHOI permission to reproduce and distribute publicly paper and electronic copies of this thesis document in whole or in part.

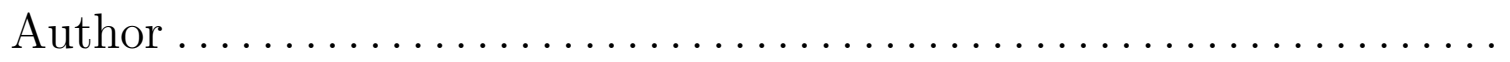

Joint Program in Physical Oceanography Massachusetts Institute of Technology Woods Hole Oceanographic Institution

May 7, 2010

Certified by .

John M. Toole

Senior Scientist

Thesis Supervisor

Accepted by

Karl Helfrich

Chair, Joint Committee for Physical Oceanography 


\title{
Near-inertial and Thermal Upper Ocean Response to Atmospheric Forcing in the North Atlantic Ocean
}

by

\author{
Katherine E. Silverthorne
}

\author{
Submitted to the Joint Program in Physical Oceanography - Massachusetts \\ Institute of Technology / Woods Hole Oceanographic Institution \\ on May 7, 2010, in partial fulfillment of the \\ requirements for the degree of \\ Doctor of Philosophy in Physical Oceanography
}

\begin{abstract}
Observational and modeling techniques are employed to investigate the thermal and inertial upper ocean response to wind and buoyancy forcing in the North Atlantic Ocean. First, the seasonal kinetic energy variability of near-inertial motions observed with a moored profiler is described. Observed wintertime enhancement and surface intensification of near-inertial kinetic energy support previous work suggesting that near-inertial motions are predominantly driven by surface forcing. The wind energy input into surface ocean near-inertial motions is estimated using the Price-WellerPinkel (PWP) one-dimensional mixed layer model. A localized depth-integrated model consisting of a wind forcing term and a dissipation parameterization is developed and shown to have skill capturing the seasonal cycle and order of magnitude of the near-inertial kinetic energy. Focusing in on wintertime storm passage, velocity and density records from drifting profiling floats (EM-APEX) and a meteorological spar buoy/tethered profiler system (ASIS/FILIS) deployed in the Gulf Stream in February 2007 as part of the CLIvar MOde water Dynamics Experiment (CLIMODE) were analyzed. Despite large surface heat loss during cold air outbreaks and the drifting nature of the instruments, changes in the upper ocean heat content were found in a mixed layer heat balance to be controlled primarily by the relative advection of temperature associated with the strong vertical shear of the Gulf Stream. Velocity records from the Gulf Stream exhibited energetic near-inertial oscillations with frequency that was shifted below the local resting inertial frequency. This depression of frequency was linked to the presence of the negative vorticity of the background horizontal current shear, implying the potential for near-inertial wave trapping in the Gulf Stream region through the mechanism described by Kunze and Sanford (1984). Three-dimensional PWP model simulations show evidence of near-inertial wave trapping in the Gulf Stream jet, and are used to quantify the resulting mixing and the effect on the stratification in the Eighteen Degree Water formation region.
\end{abstract}


Thesis Supervisor: John M. Toole

Title: Senior Scientist 


\section{Acknowledgements}

This work was supported by National Science Foundation grants OCE-0241354 and OCE-0424865, as well as the Woods Hole Oceanographic Institution's Ocean and Climate Change Institute. Funding to initiate the McLane Moored Profiler observations at Line $\mathrm{W}$ were provided by grants from the $\mathrm{G}$. Unger Vetlesen Foundation and the Comer Charitable Fund to the Woods Hole Oceanographic Institutions Ocean and Climate Change Institute.

First, I would like to thank my advisor John Toole for his years of support and infinite patience. I would also like to thank my committee members Jim Edson, Al Plueddemann, and Raf Ferrari for their contribution not only to my thesis, but to my overall development as a research scientist as well. Several scientists provided helpful discussions and guidance to me during my time at graduate school, including Steve Jayne, J.J. Park, Kurt Polzin, Jim Price, and Leif Thomas. I would like to thank Tom Farrar for his constant moral and technical support. In addition, I would like to acknowledge James Girton and Tom Sanford for graciously providing the EMAPEX floats for the CLIMODE program. Finally, I extend thanks to Steve Lentz for introducing me to the field of physical oceanography, and for providing support as my thesis defense chair.

My family is extremely important to me, and I could not have completed this work without their love and encouragement. My mom, sister Sarah, grandmother Holly, and godfather Dan are all crucial components of my support system. I'd also like to thank my best friend Colleen for always being there when I needed her. The journey through graduate school was made easier through solidarity with other Joint Program students, including Stephanie Waterman, Greg Gerbi, Beatriz Pena-Molino, Jessica Benthuysen, Hristina Hristova, Elizabeth Orchard, Kate Buckman, Annette Hynes, Chris Waters, and Kristen Whalen.

Finally, I would like to dedicate this thesis to the memory of my father, Spencer Victor Silverthorne III, who was my first physics teacher. 


\section{Contents}

1 Introduction 11

2 Seasonal kinetic energy variability of near-inertial motions* 17

2.1 Introduction . . . . . . . . . . . . . . . . . 17

2.2 Observations and analysis procedures $\ldots \ldots \ldots \ldots \ldots$

2.3 Results . . . . . . . . . . . . . . . . . . . . . . . . . . . 23

2.3.1 Representative velocity profiles and average vertical wavenumber spectra . . . . . . . . . . . . . . . . . . . 23

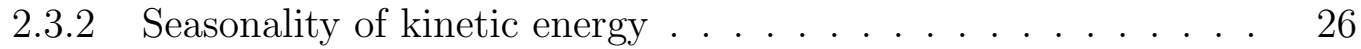

2.4 Discussion . . . . . . . . . . . . . . . . . . . . 30

3 Observations of density and velocity using drifting EM-APEX floats $\begin{array}{ll}\text { during CLIMODE } & 37\end{array}$

3.1 Introduction . . . . . . . . . . . . . . . . . . 37

3.2 Deployment details . . . . . . . . . . . . . . . . . . . . . . . . 39

3.3 Results . . . . . . . . . . . . . . . . . . . . . . . 41

3.4 Discussion . . . . . . . . . . . . . . . . . . . . . . . . 49

4 A wintertime quasi-Lagrangian heat budget in the Gulf Stream region 53

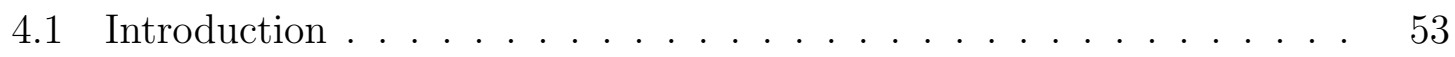

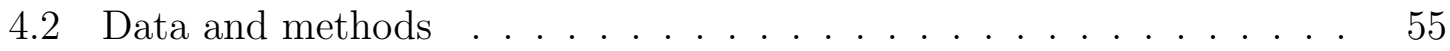

4.2 .1 Heat budget equation . . . . . . . . . . . . 55 
4.2.2 Determination of one-dimensional heat budget terms . . . . . 59

4.3 One dimensional heat budget results . . . . . . . . . . . . . 61

4.3.1 Surface forcing . . . . . . . . . . . . . 61

4.3 .2 FILIS .............................. 63

4.3 .3 EM-APEX $1633 \ldots \ldots \ldots 3$

4.3.4 EM-APEX $1636 \ldots \ldots \ldots \ldots$

4.3.5 FILIS/EM-APEX 1636 comparison . . . . . . . . . . . 65

4.3.6 PWP mixed layer model . . . . . . . . . . . . . . 66

4.4 Advective effects . . . . . . . . . . . . . . . . 68

4.5 Mixing and entrainment at the base of the mixed layer . . . . . . . . 72

4.6 Discussion . . . . . . . . . . . . . . . . . . . 75

5 The influence of near-inertial wave trapping in the Gulf Stream on $\begin{array}{ll}\text { the upper ocean stratification } & 79\end{array}$

5.1 Introduction . . . . . . . . . . . . . . . . . . . . . . 79

5.2 Model implementation . . . . . . . . . . . . . . . . 82

5.2 .1 3DPWP model . . . . . . . . . . . . . . 82

5.2 .2 Model setup . . . . . . . . . . . . . . . 83

5.2 .3 Model forcing . . . . . . . . . . . . . . . 85

5.3 Influence of relative vorticity on near-inertial motions . . . . . . 85

5.3.1 Frequency response . . . . . . . . . . . . . . 85

5.3.2 Analytical response to forcing . . . . . . . . . . . 88

5.3.3 Decay of mixed layer near-inertial kinetic energy . . . . . . . . 90

5.3.4 Vertical wavelength and mixing . . . . . . . . . . . . 93

5.4 Near-inertial kinetic energy budget . . . . . . . . . . . . . . 96

5.4.1 Formulation ................. 96

5.4 .2 Kinetic energy budget results . . . . . . . . . . . . . 97

5.5 Implications for EDW formation . . . . . . . . . . . . . . . . 99 
6 Concluding remarks $\quad 105$

6.1 Discussion . . . . . . . . . . . . . . . . . . . . 105

6.2 Future work . . . . . . . . . . . . . . . . . . . . . 108

A A linear combination near-inertial filter 111

B Estimation of the wind work on near-inertial motions 115

$\begin{array}{ll}\text { C Heat budget error calculation } & 121\end{array}$

D Analytical solution behavior as the effective inertial frequency approaches the resting inertial frequency 


\section{Chapter 1}

\section{Introduction}

The majority of the circulation in the ocean is forced either directly or indirectly by the sun and atmosphere. This forcing can take the form of solar heating, wind stress, freshwater transfer, and/or heat transfer between the ocean and atmosphere. The surface forced ocean circulation covers a large span of spatial and temporal scales; the variation of winds with latitude in all of the ocean basins results in the large-scale gyres, while mixing of wind forced internal waves helps to maintain the thermohaline abyssal circulation of the ocean. The ocean also influences the structure of the atmospheric circulation; for example, uneven solar heating of the ocean between the poles and the equator contributes to the development of the large scale atmospheric winds. Clearly, understanding the physics of the closely coupled ocean/atmosphere dynamical system is a central task for physical oceanographers. This dissertation contributes to that research by using observations and modeling to investigate the characteristics of wind-forced near-inertial motions, the upper ocean thermal response to large surface heat fluxes, and the trapping and subsequent dissipation of wind-forced nearinertial motions in a mesoscale relative vorticity field.

A substantial portion of the work presented in this thesis deals with the energetics of near-inertial motions. Herein, the term "near-inertial motions" will refer to oscillations at frequency near the rotation rate of the earth, $f$ (the inertial frequency), in which the horizontal velocity vector rotates cyclonically in time. Near-inertial oscil- 
lations are frequently observed in ocean velocity records, and the near-inertial band is often the most energetic portion of the internal wave frequency band. Near-inertial waves propagate nearly horizontally, and so can carry energy long distances in the equatorward direction from where they were generated. Internal waves are important for ocean circulation because they produce shear in the interior of the ocean and therefore are responsible for much of the mixing in the ocean interior (Polzin et al., 1995).

Although there are multiple possible energy sources for near-inertial motions (including geostrophic adjustment and parametric subharmonic instability of tidally driven motions), observational analysis suggests that the dominant one is wind forcing (Alford, 2001). Global analysis of mooring records indicates that near-inertial energy is surface intensified and enhanced during winter months, both of which are indicative of a surface source (Alford and Whitmont, 2007). Any wind stress which is variable in time can force near-inertial motions; winds which rotate at the inertial frequency produce a resonant forcing with the maximum possible energy input. Determining the pathway of wind energy imparted to the ocean is important for understanding the large-scale thermohaline circulation of the ocean. Munk and Wunsch (1998) show that the energy required to close the abyssal circulation can be accounted for by including wind and tidal forcing, with about half of that energy coming from the wind. As much as half of the wind energy imparted to the upper ocean is thought to go directly into near-inertial motions (Alford, 2001). What is still unclear, and of great importance, is the ultimate fate of that wind energy.

This issue is addressed in Chapter 2 of this thesis, where seasonal variability of near-inertial horizontal kinetic energy is examined using observations from a series of McLane Moored Profiler moorings in the western North Atlantic Ocean in combination with a one-dimensional, depth-integrated kinetic energy model. Time series of depth-dependent and depth-integrated near-inertial kinetic energy are calculated from available mooring data after filtering to isolate near-inertial-frequency motions. These data document a pronounced seasonal cycle featuring a wintertime maximum 
in the depth-integrated near-inertial kinetic energy deriving chiefly from the variability in the upper 500 meters of the water column. Rotary vertical wavenumber spectra exhibit a dominance of clockwise-with-depth energy, indicative of downward energy propagation and implying a surface energy source. A simple depth-integrated nearinertial kinetic energy model consisting of a wind forcing term and a dissipation term captures the order of magnitude of the observed near-inertial kinetic energy as well as its seasonal cycle.

The remainder of the work presented in this thesis focuses in on the wintertime upper ocean response to surface forcing in the Gulf Stream. The motivation for the work presented in Chapters 3, 4, and 5 is twofold. First, the conclusions in Chapter 2 suggest that the most energetic near-inertial motions are found during the passage of wintertime storms in the North Atlantic Ocean. Secondly, the Gulf Stream is an interesting region for air-sea interaction study because wintertime storms moving eastward off the North American continent carry cold air which contrasts sharply with the warm surface water, resulting in some of the largest surface heat fluxes anywhere in the world ocean. This ocean surface heat loss leads to vigorous convection in the upper ocean that is believed to contribute to the formation of a mode water (a large water mass characterized by weak vertical property gradients) known as North Atlantic Subtropical Mode Water, or Eighteen Degree Water (Worthington, 1959). Discrepancies in observations of rates of Eighteen Degree Water formation from direct float observations (Kwon and Riser, 2004a) and from air-sea buoyancy flux estimates (Speer and Tziperman, 1992) motivated the CLIvar MOde water Dynamics Experiment (CLIMODE), a large scale observational program designed to provide a variety of data describing the wintertime formation process. The observations presented in Chapters 3 and 4 were collected as part of the CLIMODE program.

Chapter 3 describes observations from three separate sources: two drifting profiling floats (EM-APEX) and an air-sea interaction buoy tethered to a profiling current meter and CTD system (ASIS-FILIS). All of these instruments were deployed in February - March of 2007 in the Gulf Stream region. The meteorological data re- 
turned from both the drifting buoy and shipboard cruise data showed energetic wind stress and large ocean surface heat loss that were typical of winter conditions in this region. The temperature records from the EM-APEX and ASIS-FILIS systems displayed evidence that newly-formed Eighteen Degree Water was sampled during the deployments. In addition, velocity records from the EM-APEX floats contained energetic near-inertial motions in the Gulf Stream region. Shifting of the inertial frequency below that for a resting ocean is also described, and related to the mesoscale horizontal shear of the Gulf Stream.

The response of the upper ocean to the strong buoyancy forcing is evaluated in Chapter 4 through the implementation of a mixed layer heat balance study applied to the EM-APEX float and ASIS-FILIS data. Using this framework, the observed changes in upper ocean heat content can be related to the surface forcing, advection, and mixing processes. This analysis supports one of the CLIMODE program's primary goals, tying the surface forcing directly to the upper ocean evolution. Because the floats were drifting with the background flow, the initial expected outcome of the heat balance was a very close connection between the surface buoyancy forcing and the upper ocean heat content. However, subsequent detailed analysis showed that the vertical motion of the profilers made them susceptible to advective effects, due in large part to the vertical shear of the Gulf Stream. Consequently, the observed mixed layer depth evolution was not able to be directly linked to the surface forcing.

The work presented in Chapter 5 of this thesis was motivated by the previously mentioned float observations which indicated that the near-inertial frequency was modified by the relative vorticity of the mesoscale flow. Previous studies have shown that near-inertial motions can exhibit unusual behavior in the presence of a strong vorticity signal, particularly negative vorticity (Kunze and Sanford, 1984). Observations and model studies suggest that mixed layer near-inertial oscillations decay rapidly in the presence of a strong relative vorticity gradient (Meurs, 1998; Balmforth and Young, 1999). Below the mixed layer, near-inertial internal waves can encounter horizontal turning points and approach vertical critical layers in negative 
vorticity regions. This can lead to enhanced dissipation near vertical critical layers, as the near-inertial energy is unable to propagate out of the negative vorticity region (Kunze, 1985). This trapping mechanism therefore provides a source of mixing in the Eighteen Degree Water formation region which could act in concert with or counteract the primary formation mechanism (convection due to the surface heat loss). The impact of the mixing of trapped near-inertial waves on the upper ocean stratification is evaluated by simulating a Gulf-Stream-like jet system with a numerical model. The model domain is wind forced, and the resulting near-inertial energy propagation is described. Weakened stratification associated with near-inertial wave trapping is found, but this preconditioning for mode water formation is complicated by processes which act to restratify the surface mixed layer in the negative vorticity region. 


\section{Chapter 2}

\section{Seasonal kinetic energy variability of near-inertial motions*}

\section{$2.1 \quad$ Introduction}

Oscillations about the inertial frequency, $f$, are a commonplace feature of the ocean with frequency spectra of most ocean current records tending to display an energetic peak associated with these motions, Figure 2-1. The predominant generation mechanism for near-inertial motions is thought to be wind forcing at the ocean surface (Pollard and Millard, 1970; D'Asaro, 1985); near-inertial motions can also be forced by geostrophic adjustment. Near-inertial internal waves have a nearly horizontal group velocity, and thus can propagate energy long distances without encountering the ocean surface or bottom (Garrett, 2001). However, wave-wave interactions can lead to energy fluxes into and/or out of the near-inertial frequency band, and dissipation can occur which acts as a sink for near-inertial energy.

In this paper, we investigate the seasonality of near-inertial internal waves at a site

*This chapter is based on the paper "Seasonal Kinetic Energy Variability of Near-Inertial Motions" by K. E. Silverthorne and J. M. Toole, J. Phys. Oceanogr., vol. 39, pp. 1035-1049, 2009. (c)American Meteorological Society. Reprinted with permission. 


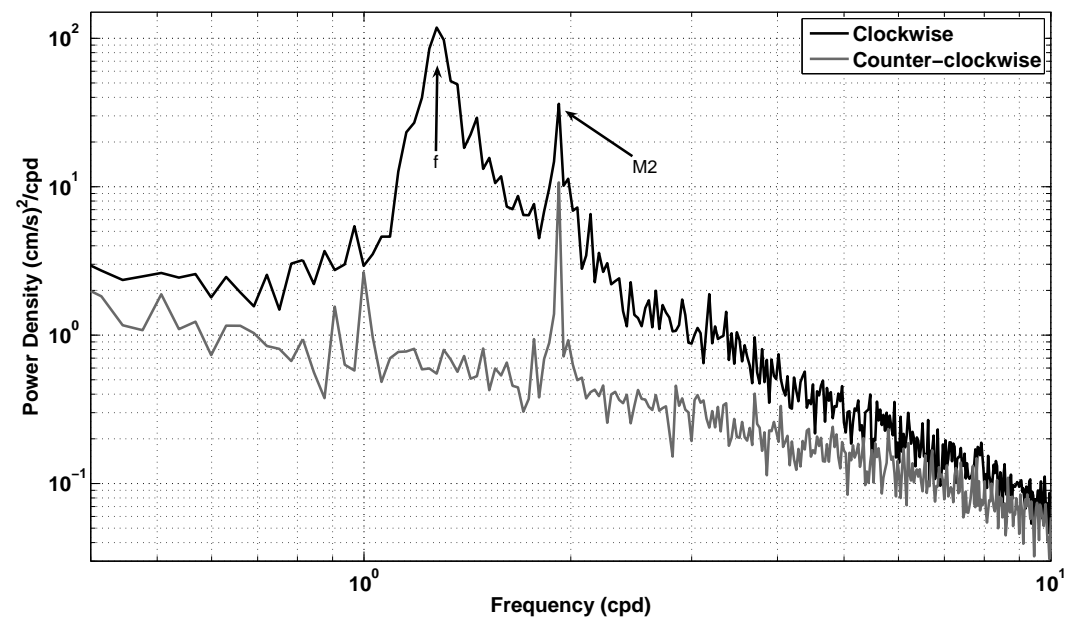

Figure 2-1: Rotary frequency spectrum of a Line $\mathrm{W}$ vector averaging current meter record from $39.2^{\circ} \mathrm{N}, 69.4^{\circ} \mathrm{W}$ and 1000 meters depth spanning the time period April 2004 to April 2006. The clockwise and counterclockwise components of the spectrum are black and gray, respectively. The spectrum was derived by averaging each periodogram value over 21 neighboring frequency estimates. The inertial peak (frequency $=1.3$ cycles per day) and the M2 tidal peak (frequency $=1.9$ cycles per day) are marked.

in the western North Atlantic Ocean and examine a localized kinetic energy budget for these motions, focusing specifically on the wind energy input. Because near-inertial motions are so energetic, investigating the near-inertial kinetic energy budget is important from a global perspective. Munk and Wunsch (1998) showed that substantial amounts of energy are needed from the winds and tides to drive the mixing required to maintain the abyssal stratification of the ocean. It has been estimated that the global wind work on inertial motions is comparable to the wind work on geostrophic motions (Alford, 2001, 2003). Therefore, investigating the relative importance of the terms in the near-inertial kinetic energy budget contributes to the understanding of the global oceanic energy budget.

Excitation of near-inertial motions by wind forcing has been examined extensively in both modeling and observational studies (Pollard and Millard, 1970; D'Asaro, 1985; Plueddemann and Farrar, 2006). Time-varying wind stress on the ocean surface can 
drive inertial mixed layer currents that, in the presence of spatial inhomogeneities of these currents, can force downward propagating near-inertial internal waves at the base of the mixed layer. D'Asaro (1985) showed that wind forcing of inertial motions is caused primarily by the passage of storms such as cold fronts and small low pressure systems. Accordingly, there is commonly a winter maximum in the wind energy input into near-inertial motions because of the prevalence of storms in this season (Alford, 2001). Recent work by Alford and Whitmont (2007) examined temporal and spatial patterns of near-inertial kinetic energy observed at current meter moorings across the globe. They concluded that there is a surface-intensified, seasonal cycle in nearinertial kinetic energy that is correlated with the estimated wind forcing.

Generation of near-inertial motions by wind forcing has traditionally been studied using the slab model developed by Pollard and Millard (1970). Recent studies have shown that the slab model can overestimate the work done by the wind due to its simple linear parameterization of dissipative processes at the base of the mixed layer (Plueddemann and Farrar, 2006). The Price-Weller-Pinkel (PWP) mixed layer model appears to give a better estimate of the work done by the wind because it includes a transition layer below a slab-like mixed layer (Price et al., 1986). Vertical transfers of momentum and energy by turbulent mixing governed by a Richardson number criterion and by convection are simulated in the PWP model, allowing the mixed layer depth to evolve, thus making it more realistic than the slab model.

As noted earlier, one possible sink for the energy put into near-inertial motions by the wind is turbulent dissipation due to the interactions of internal waves. Polzin et al. (1995) compared dissipation predictions based on wave-wave interaction models with observations and showed that finescale parameterization of dissipation in terms of properties of the internal wave field can accurately capture observations in regions where internal waves are thought to dominate the mixing (i.e. away from boundaries and large current shear). In particular, the results of their study confirmed the dependence of the dissipation on the buoyancy frequency and the energy level of the internal wave field. 
Depth-dependent and depth-integrated time series of near-inertial kinetic energy levels are calculated in this study from observations in the western North Atlantic at $39^{\circ} \mathrm{N}$ over a 5 year period. The near-inertial kinetic energy exhibits a strong seasonal cycle with a wintertime maximum, and is dominated by downward energy propagation. A simple kinetic energy model is constructed, and the model results are compared to the observations. It is shown that the kinetic energy model captures the magnitude and seasonal cycle of the near-inertial kinetic energy levels seen in observations.

\subsection{Observations and analysis procedures}

Observations used to calculate the near-inertial horizontal kinetic energy were obtained from a series of moorings fitted with McLane Moored Profilers (MMPs) located at approximately $38.8^{\circ} \mathrm{N}$ and $69.2^{\circ} \mathrm{W}$ in the western North Atlantic Ocean as part of the Line W project, Figure 2-2 (see website: www.whoi.edu/science/PO/linew/). The details of the MMP deployments are found in Table 2.1. The MMPs (Doherty et al., 1999; Toole et al., 1999; Morrison et al., 2000) were programmed to sample in bursts of four near-full-depth (one-way) profiles, with initiation of each profile in

Table 2.1: MMP deployment details

\begin{tabular}{cc}
\hline \hline Location & $38.8^{\circ} \mathrm{N}, 69.2^{\circ} \mathrm{W}$ \\
Water depth $(\mathrm{m})$ & $\sim 3100$ \\
Instrumentation & CTD: temperature, salinity, and pressure \\
& Zonal and meridional velocities \\
Vertical range $(\mathrm{m})$ & $75-3000$ \\
Vertical resolution (dbar) & 2 \\
Temporal range & Four deployments: \\
& 3 Nov. $2001-14$ Aug. 2002 \\
& 5 Jun. $2003-1$ May 2004 \\
& 10 May $2004-16$ Mar. 2005 \\
& 10 May $2005-5$ Apr. 2006 \\
Temporal resolution & Burst of four inertial profiles separated by half an \\
& inertial period (9.5 hr); \\
bursts separated by $4-5$ days
\end{tabular}




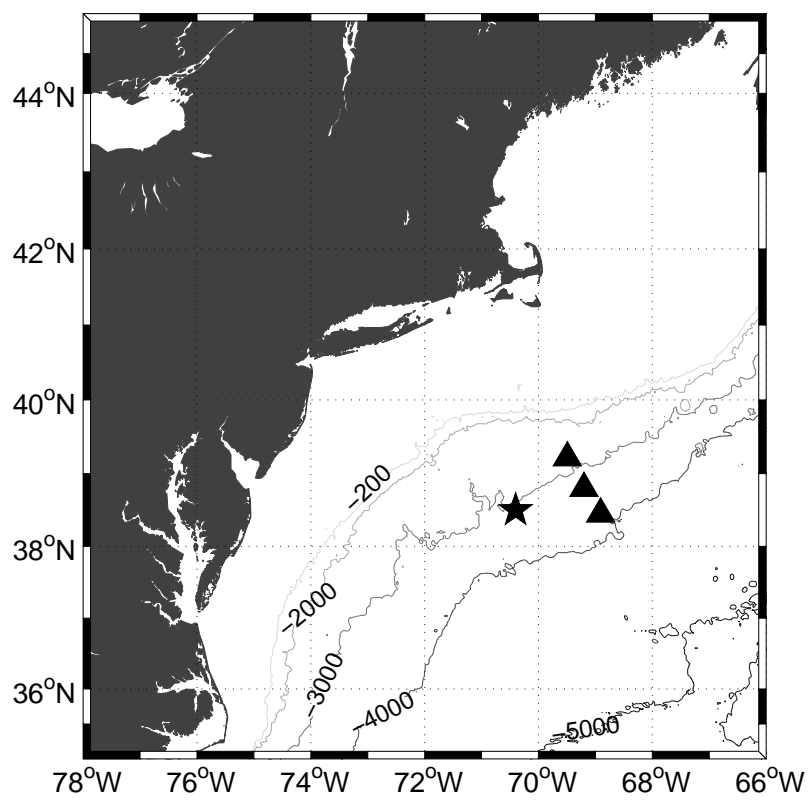

Figure 2-2: Map of Line W mooring (triangles) and NDBC buoy (star) locations. The top and bottom triangles mark current meter moorings (W2 and W4 respectively) while the middle triangle indicates the MMP mooring location. Selected isobaths (meters) are also displayed.

a burst being separated by half an inertial period and successive bursts separated by 4-5 days. Nominal MMP profile speed is $0.25 \mathrm{~ms}^{-1}$. During each MMP profile, temperature, conductivity and pressure data are acquired at approximately $1.6 \mathrm{~Hz}$ using a low-power CTD and 3-axis velocity and geographic heading data are obtained at comparable rate by an acoustic-travel-time current meter. These raw data were reduced during post-processing to $2 d b$ bin-averaged estimates of temperature, salinity and velocity.

Several steps were taken in the analysis of the MMP velocity data in order to extract estimates of the near-inertial kinetic energy. At each depth, the average velocity of the four profiles in each burst was removed to suppress the low-frequency flow signals. The depth-mean of each velocity anomaly profile was also removed. 
The resulting anomaly profiles are thus dominated by high-frequency, depth varying motions believed to consist chiefly of internal waves, and are referred to as "superinertial motions" in the following. Estimates of the near-inertial horizontal velocity profile $\left(u_{i}, v_{i}\right)$ for each burst was derived from a linear combination of the four anomaly profiles as follows:

$$
\begin{aligned}
& u_{i}=.25\left(u_{1}-u_{2}+u_{3}-u_{4}\right) \\
& v_{i}=.25\left(v_{1}-v_{2}+v_{3}-v_{4}\right)
\end{aligned}
$$

where $\left(u_{1}, v_{1}\right)$ is the first velocity anomaly profile in a burst, $\left(u_{2}, v_{2}\right)$ is the second velocity anomaly profile in the burst, and so on. This inertial filtering technique, discussed in detail in Appendix A, takes advantage of the fact that the ratio of the inertial period to the M2 tidal period at the mooring latitude is 1.5 to filter semi-diurnal tidal energy. The near-inertial kinetic energy profile for each burst was calculated as follows:

$$
K E_{i}=\frac{1}{2} \rho_{0}\left(u_{i}^{2}+v_{i}^{2}\right)
$$

When performing depth integration of the near-inertial kinetic energy, missing values (as occur when the MMP fails to profile fully between the top and bottom stops on the mooring wire) were assumed to be zero, thus yielding a lower-bound estimation.

Because the near-inertial kinetic energy is expected to vary with the buoyancy frequency, $N$, to first order, it is necessary to apply WKB-scaling to remove this dependence (Leaman and Sanford, 1975). The super-inertial and near-inertial kinetic energy profiles were therefore WKB-scaled using buoyancy frequency estimates derived from time-averaged MMP measurements of temperature, salinity, and pressure, and the standard $N_{o}$ value of $3 \mathrm{cph}$. Rotary vertical wavenumber spectra of the WKB-scaled velocity profiles were calculated for the depth range of 200 to 500 meters to infer the sense of vertical energy propagation. The mean value of the buoyancy frequency was $2 \mathrm{cph}$ and varied little over that depth range. Examination of vertical wavenumber spectra of the full MMP observed velocity profiles during summertime indicated that the spectra began to level out (indicating noise) at vertical wavelengths 
smaller than 10 meters. Integrating this inferred noise level across wavenumber space gives an estimate of the MMP RMS velocity error as $0.7 \mathrm{cms}^{-1}$.

\section{$2.3 \quad$ Results}

\subsubsection{Representative velocity profiles and average vertical wavenumber spectra}

The derived super-inertial anomaly velocity profiles often display the mirror imaging previously observed in velocity measurements separated by half an inertial period, Figure 2-3. Clockwise rotation with depth of the near-inertial velocity vector is evident in the estimated near-inertial velocity components, Figure 2-3 (right column). Of the three examples presented, the typical summer time velocity profiles are the smallest in magnitude, and also do not exhibit much variation with depth, Figure 2-3 (top row). In contrast, the typical winter time velocity profiles display a strong intensification in the upper 500 meters of the water column, Figure 2-3 (middle row). Velocity profiles estimated during the winter of 2001-2002 demonstrate unusually large magnitude at depths below 500 meters, Figure 2-3 (bottom row).

The average vertical wavenumber kinetic energy and shear spectra based on all available velocity anomaly profiles (Figures 2-4 and 2-5) are in good agreement with the Garrett-Munk model (Munk, 1981). The shape of the near-inertial kinetic energy spectrum closely parallels that of the full super-inertial spectrum and accounts for much of the magnitude of the latter. Both the super-inertial and the near-inertial vertical wavenumber shear spectra are blue for vertical wavenumbers less than $10^{-2}$ cpm and nearly white over the vertical wavenumber range from $10^{-2}$ to $10^{-1} \mathrm{cpm}$, with the spectra being dominated by noise at vertical wavenumbers greater than $10^{-1}$ cpm. Rotary vertical wavenumber spectra of the WKB-scaled near-inertial velocity show that the near-inertial motions are dominated by clockwise turning with depth motions at vertical scales larger than 100 meters, Figure 2-6. (Clockwise turning is 

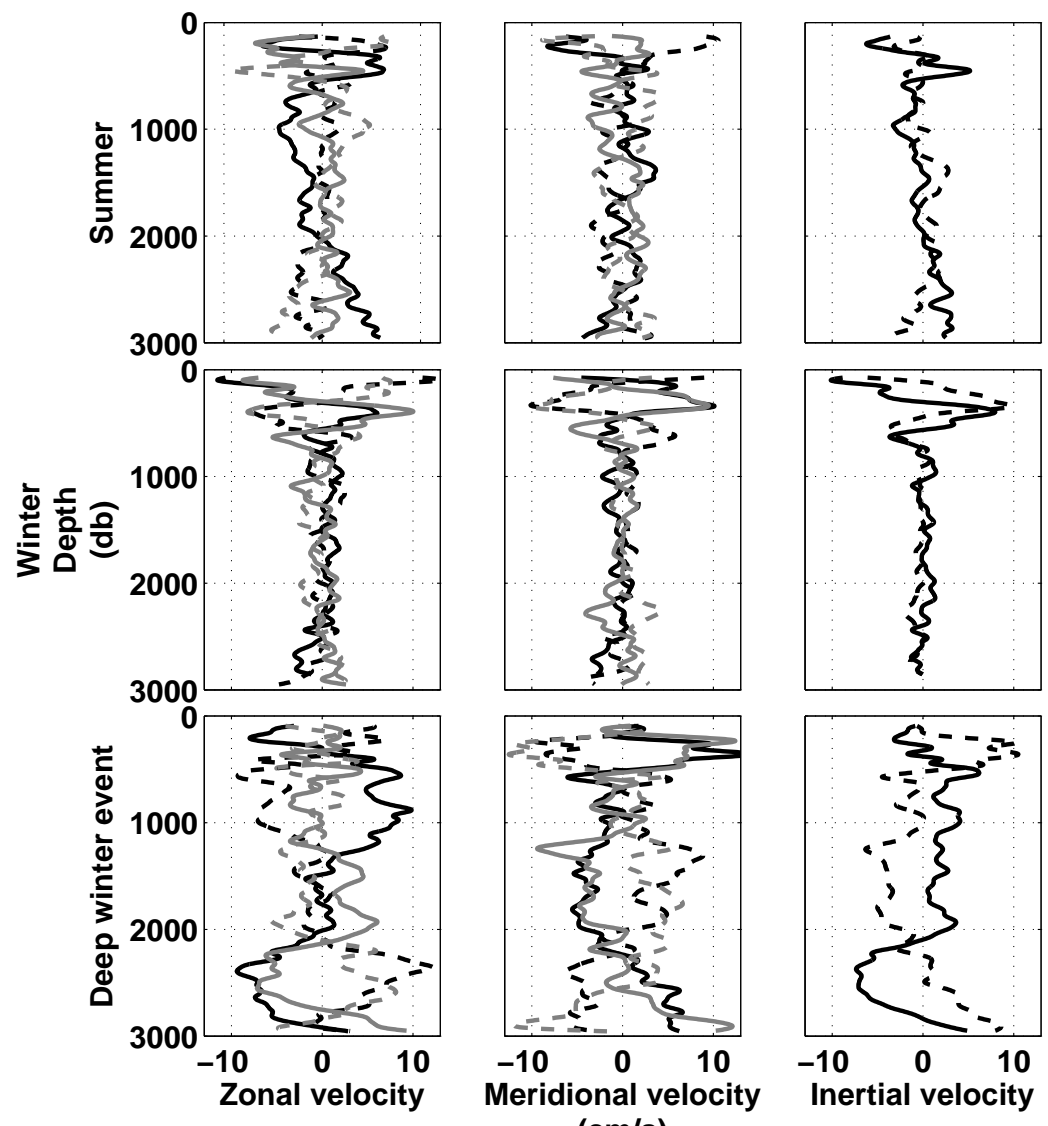

$(\mathbf{c m} / \mathbf{s})$

Figure 2-3: MMP anomaly velocity depth profiles in $\mathrm{cms}^{-1}$ which have been smoothed with a running depth mean of 50 meters. The top row of panels show data from a typical summer burst (observed in June 2002), the middle row of panels show data from a typical winter burst (observed in January 2004), and the bottom row of panels show data from the anomalous winter deep event (observed in February 2002). The left panels show the zonal velocities and the middle panels show the meridional velocities where the solid black line represents the first burst profile, the dashed black line represents the second burst profile, the solid gray line represents the third burst profile, and the dashed gray line represents the fourth burst profile. The right panels show the zonal (solid black line) and meridional (dashed black line) inertial velocity components as calculated using the linear combination scheme.

indicative of the dominance of low-frequency internal waves carrying energy downward.) This assessment holds true for both summertime and wintertime data subsets. 


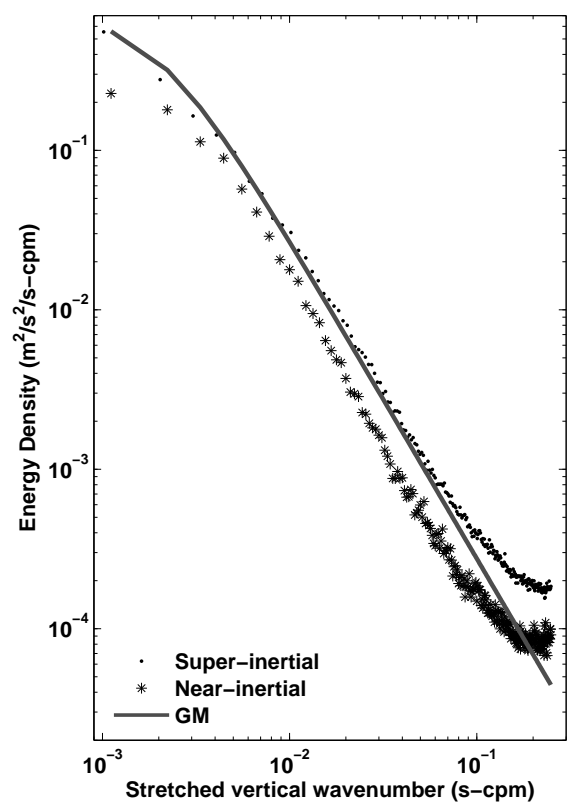

Figure 2-4: Observed WKB-scaled super-inertial (diamonds) and near-inertial (stars) vertical wavenumber kinetic energy spectra with the Garrett-Munk model (gray line) for jstar $=5$. The super-inertial vertical wavenumber kinetic energy spectrum was calculated as the mean of periodograms estimated from 360 velocity anomaly profiles, while the near-inertial kinetic energy vertical wavenumber spectrum was calculated as the mean of periodograms derived from 78 near-inertial velocity profiles.

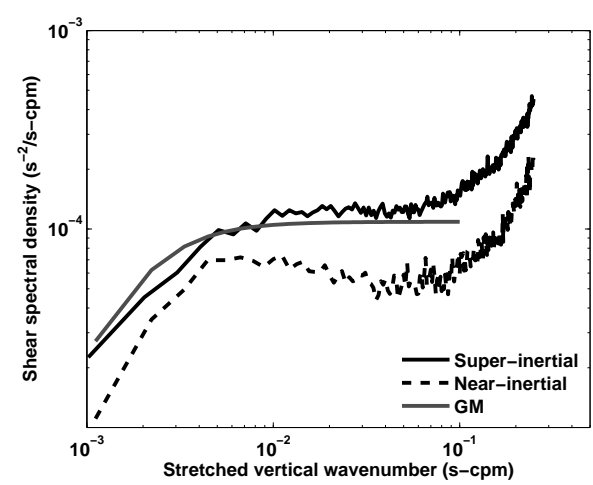

Figure 2-5: Observed WKB-scaled super-inertial (black line) and near-inertial (black dotted line) vertical wavenumber shear spectra with the Garrett Munk model (gray line) for jstar $=5$. The shear spectra were derived as the product of the velocity spectra (Fig. 2-4) and the square of the vertical wavenumber. 

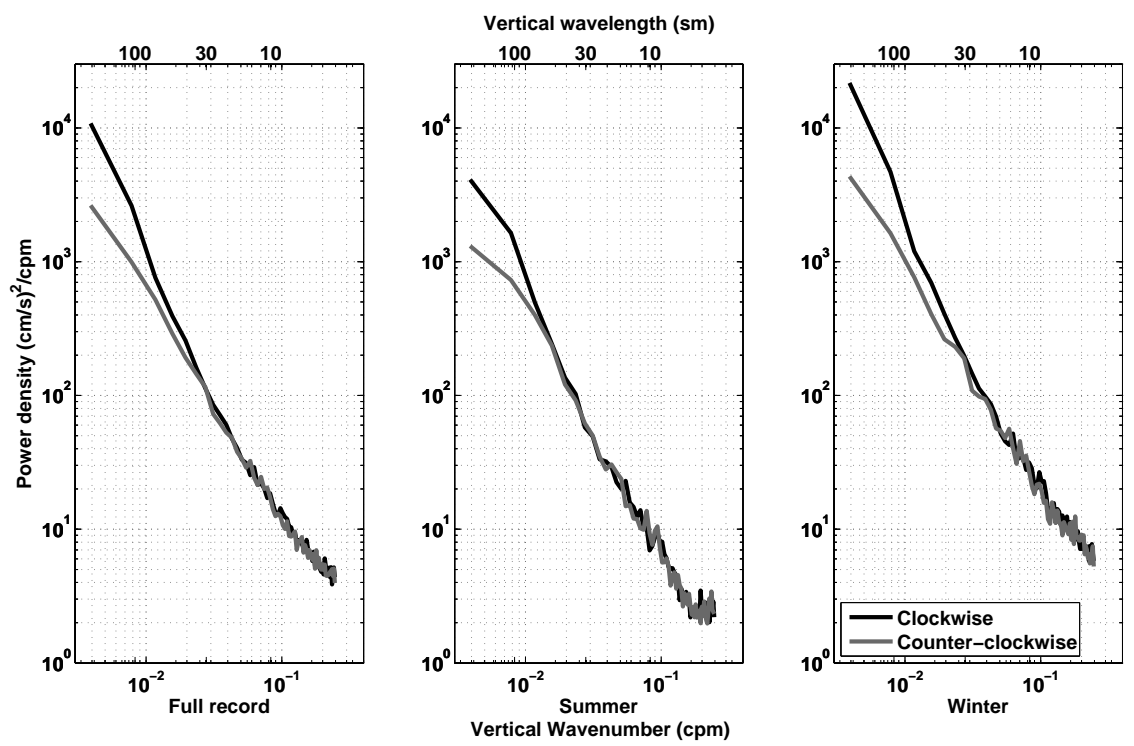

Figure 2-6: Rotary vertical wavenumber spectra of the WKB scaled near-inertial velocity profiles showing the clockwise turning with depth component (black line) and the counterclockwise component (gray line). The full near-inertial kinetic energy spectrum(left panel), summertime near-inertial kinetic energy spectrum(middle panel), and wintertime near-inertial kinetic energy spectrum (right panel) were calculated as the mean of 214, 63, and 66 velocity periodograms, respectively, for the depth interval of 200 to 500 meters. The top axis shows the WKB-stretched vertical wavelength derived using a $N_{o}$ value of $3 \mathrm{cph}$.

However, the clockwise wintertime near-inertial motions contain more energy at larger vertical scales than the summertime clockwise near-inertial motions.

\subsubsection{Seasonality of kinetic energy}

Consistent with expectations based on prior work, a seasonal cycle of the near-inertial motions is observed with enhanced depth-integrated kinetic energy in winter and minimum energy in summer, Figure 2-7. Depth-time contour plots of the super- and near-inertial kinetic energy (Figures 2-8 and 2-9) reveal that the bulk of the winter enhancement of depth-integrated super-inertial and near-inertial kinetic energy derives from depths less than 500 meters: the principal exception being the strong 


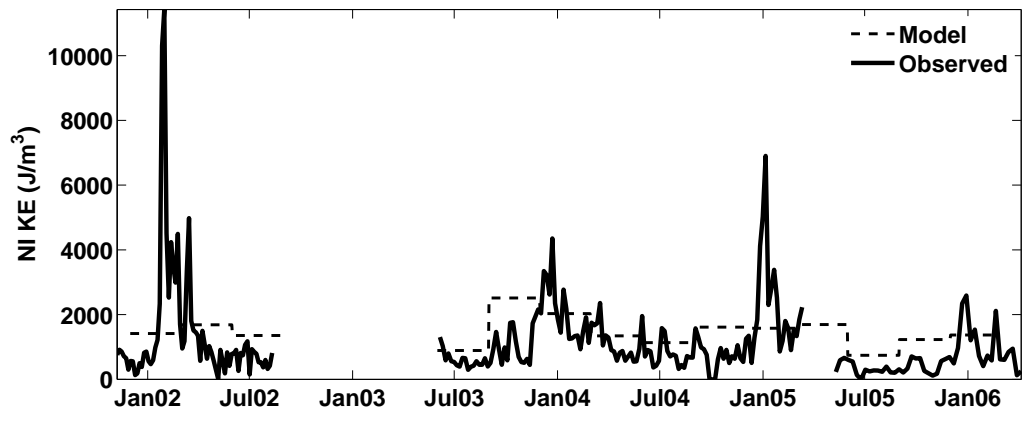

Figure 2-7: Depth integrated near-inertial kinetic energy from observations (black line) and model (thin-dashedline) in $\mathrm{Jm}^{-3}$. The model results shown are seasonal averages.

event in the winter of 2001-2002 which reached below 3000 meters. Observations

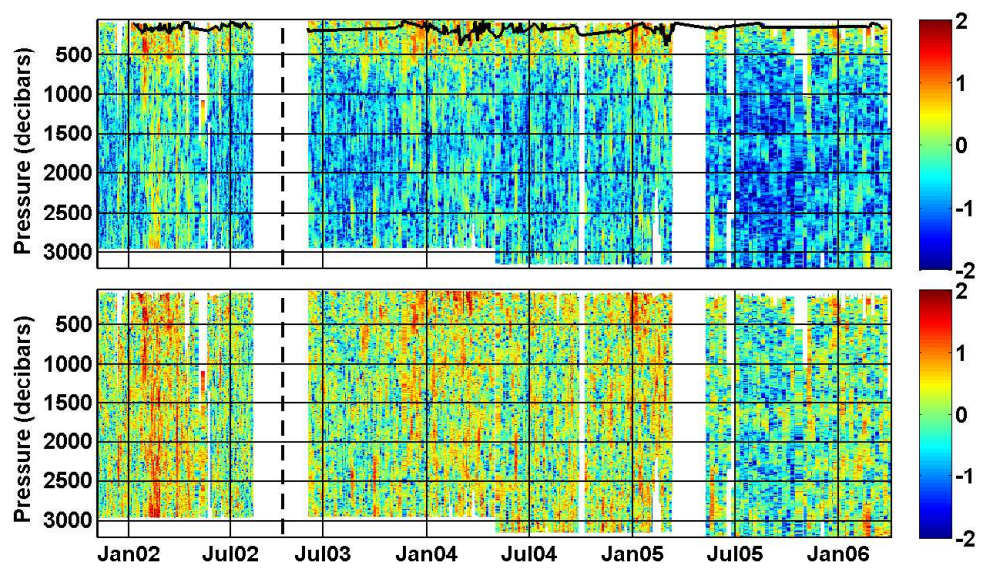

Figure 2-8: Top panel: Depth-time contour plot of the log of the observed superinertial kinetic energy in $\mathrm{Jm}^{-3}$. The black dots represent the MMP observed mixed layer depth (when available). Bottom panel: Depth-time contour plot of the log of the WKB-scaled observed super-inertial kinetic energy in $\mathrm{Jm}^{-3}$. The black dotted lines mark a jump in the time axis.

of the mixed layer depth (when available) demonstrate that the wintertime maxima 


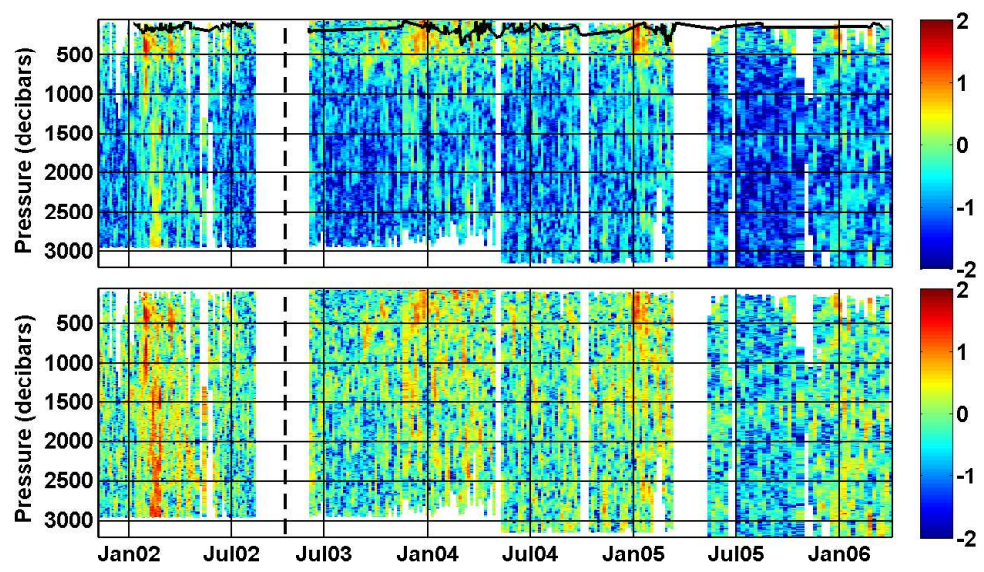

Figure 2-9: Top panel: Depth-time contour plot of the log of the observed nearinertial kinetic energy in $\mathrm{Jm}^{-3}$. The black dots represent the MMP observed mixed layer depth (when available). Bottom panel: Depth-time contour plot of the log of the WKB-scaled observed near-inertial kinetic energy in $\mathrm{Jm}^{-3}$. The black dotted lines mark a jump in the time axis.

of near-inertial kinetic energy consistently extended well below the mixed layer base. Although the WKB-scaled super- and near-inertial kinetic energies contain less variation with depth than their unscaled counterparts, they are nevertheless dominated by the strong, surface-intensified seasonal pulses, Figures 2-8 and 2-9 (bottom panels). The wintertime enhancement and surface intensification can be seen more clearly in seasonally-averaged profiles of observed super-inertial and near-inertial kinetic energy, Figures 2-10 and 2-11. The time-averaged near-inertial kinetic energy profile is similar in shape and only slightly smaller in magnitude than the total super-inertial kinetic energy profile. The summertime near-inertial kinetic energy profile is nearly uniform with depth, whereas the wintertime near-inertial kinetic energy displays statisticallysignified enhancement in the upper portion of the water column. (Confidence bounds at the 95 percent significance level were derived based on the 10-day decorrelation time scale for the inter-seasonal kinetic energy time series suggested by analysis of 

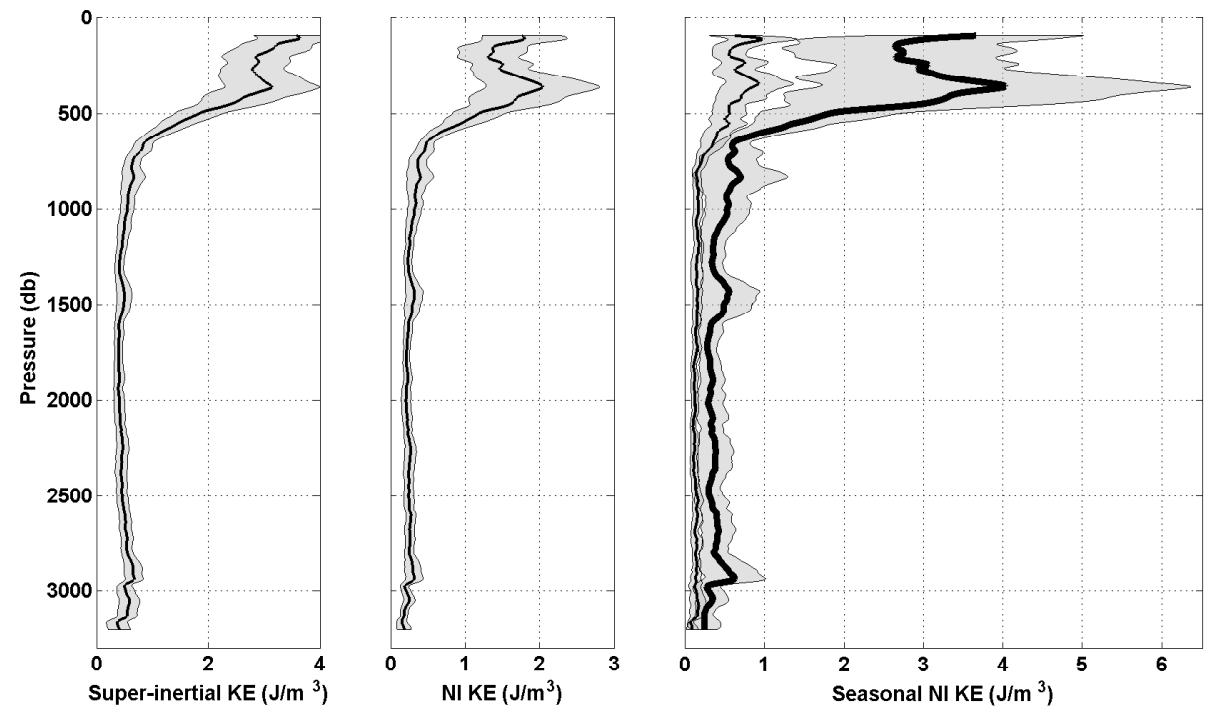

Figure 2-10: Observed time mean kinetic energy profiles with 95\% confidence intervals calculated by assuming using a decorrelation timescale of 10 days. Profiles have been smoothed with a running mean over 20 meters. The left panel shows the super-inertial kinetic energy profile, the middle panel shows the near-inertial kinetic energy profile, and the right panel shows the summertime mean (thinner black line) and wintertime mean (thicker black line). The summertime mean was derived using values from June, July, and August, while the wintertime mean was derived using values from December, January, and February.

autocorrelation functions.) Alford and Whitmont (2007) observed surface-intensified enhancement of wintertime WKB-scaled near-inertial kinetic energy with a decay in energy from 500 meters to 3500 meters depth by a factor of 3-4; here we observe a decay of approximately a factor of 2 from 500 meters depth to 3200 meters depth. In contrast, the WKB-scaled near-inertial kinetic energy in January through March of 2002 was nearly constant in depth due to anomalous deep energy. The enhanced near-inertial energy below 1200 meters depth appeared approximately 2 weeks after the start of a surface intensified energy pulse and lasted for over 2 weeks. 

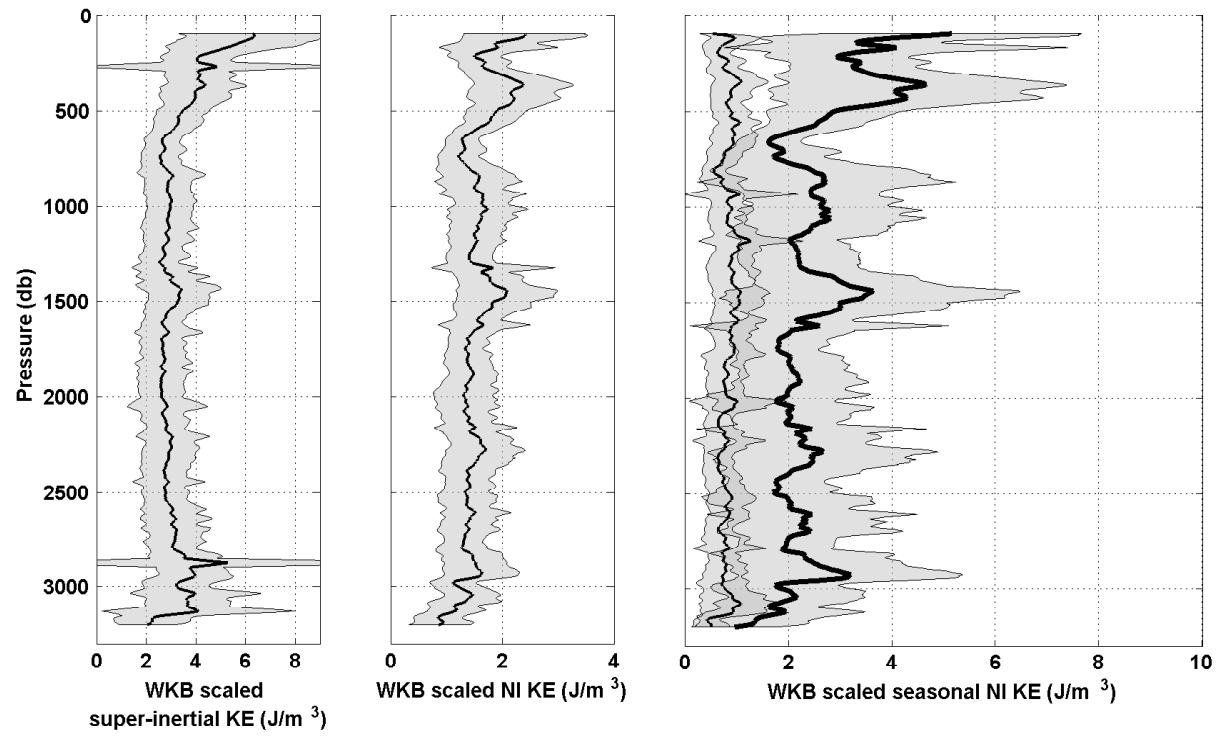

Figure 2-11: WKB-scaled time mean kinetic energy profiles with $95 \%$ confidence intervals calculated using a decorrelation timescale of 10 days. Profiles have been smoothed with a running mean over 20 meters. The left panel is the super-inertial kinetic energy profile, the middle panel shows the near-inertial kinetic energy profile, and the right panel shows the summertime mean (thinner black line) and wintertime mean (thicker black line). The summertime mean was derived using values from June, July, and August, while the wintertime mean was derived using values from December, January, and February.

\subsection{Discussion}

Examination of the observed super-inertial and near-inertial kinetic energy reveals the following notable feature: a marked seasonal cycle with a winter maximum most pronounced in the upper 500 meters of the water column. The surface intensification of the near-inertial kinetic energy, along with the dominance of downward propagating near-inertial energy at large vertical scales, endorse the hypothesis that near-inertial internal waves in this region are chiefly surface forced. Furthermore, the seasonal cycle in the observed near-inertial kinetic energy supports the idea that the near- 
inertial motions are predominantly forced by the passage of winter storms, as was concluded by Alford and Whitmont (2007).

To further develop the idea that winter storms are responsible for the observed seasonal signal, we constructed a simple local kinetic energy model for the near-inertial motions. Invoking an eddy-viscosity closure approximation, the horizontal momentum equations were band-passed over the near-inertial frequency band, yielding the following:

$$
\begin{aligned}
\frac{\partial}{\partial t} u_{i}+\langle\mathbf{u} \cdot \nabla u\rangle-f v_{i} & =-\frac{1}{\rho_{0}} \frac{\partial}{\partial x} P_{i}+A \frac{\partial^{2}}{\partial z^{2}} u_{i} \\
\frac{\partial}{\partial t} v_{i}+\langle\mathbf{u} \cdot \nabla v\rangle+f u_{i} & =-\frac{1}{\rho_{0}} \frac{\partial}{\partial y} P_{i}+A \frac{\partial^{2}}{\partial z^{2}} v_{i}
\end{aligned}
$$

where $A$ is the eddy viscosity, $\left(u_{i}, v_{i}\right)$ is the near-inertial velocity, $P_{i}$ is the near-inertial pressure, and the operator \langle\rangle represents the near-inertial band-pass filter. Equations 2.4 and 2.5 were then multiplied by the zonal and meridional components of nearinertial velocity respectively, added together, multiplied by density, and integrated in depth to produce an equation governing the near-inertial kinetic energy:

$$
\begin{aligned}
\underbrace{\frac{\partial}{\partial t} \int_{-B}^{0} K E_{i} d z}_{I}= & \underbrace{\mathbf{u}_{i} \cdot \tau_{i}}_{I I}-\underbrace{\int_{-B}^{0} \nabla \cdot\left(\mathbf{u}_{i} P_{i}\right) d z}_{I I I} \\
& -\underbrace{\rho_{0} \int_{-B}^{0}\left(u_{i}\langle\mathbf{u} \cdot \nabla u\rangle+v_{i}\langle\mathbf{v} \cdot \nabla v\rangle\right) d z}_{I V} \\
& -\underbrace{\left.A \int^{2}\right]}_{V=\int_{-B}^{0}\left[\left(\frac{\partial u_{i}}{\partial z}\right)^{2}+\left(\frac{\partial v_{i}}{\partial z}\right)^{2}\right] d z}
\end{aligned}
$$

Here $K E_{i}$ is the near-inertial kinetic energy, $B$ represents the water depth, and the near-inertial band-pass-filtered wind stress $\tau_{i}$ has been introduced using the expression: $\rho_{0} A\left(\frac{\partial u_{i}}{\partial z}, \frac{\partial v_{i}}{\partial z}\right)=\tau_{i}$. Also, bottom stresses have been assumed to be small compared to the other terms. Neither the spatial nor the temporal resolution of the early Line $\mathrm{W}$ moored measurements were sufficient to estimate the phase of the inertial motions or horizontal gradients, so the energy flux divergence term $(I I I)$ could not 
be estimated. If the near-inertial internal wave energy consists of bursts of wave packets, one might expect that the energy flux divergence term during individual storm events would not be negligible as these packets propagated past the MMP mooring. Indeed, (D'Asaro et al., 1995) showed that during the Ocean Storms experiment the observed decay of near-inertial energy out of a region after a storm was consistent with the predicted horizontal internal wave propagation. However, given a random forcing and wave field, the average horizontal energy flux at long time scales (and in turn its divergence) should be close to zero. One possible flaw in this reasoning is the location of the MMP on the continental slope, approximately $100 \mathrm{~km}$ from the shelf break, which could mean that the internal wave field has a preferred direction and the energy flux divergence does not average out to zero.

The wind work term (II) was estimated using the PWP mixed layer model as described in Appendix B, and introduced into the kinetic energy model as a prescribed forcing term. Term $I V$ on the right hand side of the kinetic energy equation represents the transfer of energy between the near-inertial band and motions of other frequencies, while term $V$ takes the form of a viscous dissipation, with the parameterized eddy viscosity $(A)$ in place of the traditional molecular viscosity. Focusing on vertical scales of order $100 \mathrm{~m}$ (which characterize the observed seasonal near-inertial signal), we neglect term $V$ with respect to term $I V$, arguing that the latter represents the principal transfer of energy to smaller vertical scales where it is presumed to dissipate. (However, term $V$ may contribute in the surface mixed layer, see below.) The wavewave interaction term was parameterized as a turbulent dissipation in terms of the kinetic energy itself, making the model nonlinear. The near-inertial kinetic energy was initially set to zero, and the model was stepped forward in time using a forward difference scheme at an hourly time step, solving for the depth-integrated kinetic energy $(I)$.

Random superposition of internal waves can lead to enhanced shear levels which are associated with wave breaking and subsequent mixing, and therefore the turbulent dissipation due to wave-wave interactions is dependent on the characteristics of 
the internal wave field. Gregg (1989) and Polzin et al. (1995) determined that the turbulent kinetic energy dissipation rate, $\epsilon$, can be expressed in terms of the buoyancy frequency $N$, base level kinetic energy $E_{o}$, and the internal wave energy level $E$ as

$$
\epsilon=7 \times 10^{-10}\left(\frac{N}{N_{o}}\right)^{2}\left(\frac{E}{E_{o}}\right)^{2}\left(W k g^{-1}\right)
$$

which is consistent with the dynamical models introduced by Henyey et al. (1986); McComas and Muller (1981). This equation was substituted for term $I V$ in the near-inertial kinetic energy equation (2.6) under the assumption that the dissipation is local in frequency space, i.e. that the loss rate of near-inertial kinetic energy by wave-wave interactions is dependent on the near-inertial internal wave energy level. Inspection of the MMP observations indicates that the near-inertial and the superinertial kinetic energy have similar shape in vertical wavenumber space and variations in time implying that they are proportional. Therefore, the parameterization of the dissipation of near-inertial kinetic energy as in equation 2.7 is consistent with the behavior of the observed internal wave field. The buoyancy frequency, $N$, in equation 2.7 was calculated as the time and depth mean of the buoyancy frequency for all of the MMP profiles, and the base level kinetic energy $E_{o}$ was taken as the summertime (June, July, and August) mean of the observed depth integrated near-inertial kinetic energy.

The depth integrated near-inertial kinetic energy model (consisting of the wind input term given as $\mathbf{u}_{i} \cdot \tau_{i}$ and a dissipation term governed by widely accepted finescale parameterization of internal wave decay) gives a prediction for seasonal-mean KE that is within a factor of 2.5 of the observations and also captures the observed wintertime enhancement, Figure 2-7. Inspection of the individual terms of the kinetic energy model showed that at long periods, the time rate of change of the depth integrated near-inertial kinetic energy is small, and therefore the dissipation term is nearly equal and opposite to the wind forcing term on seasonal time scales. The ability of the depth-integrated near-inertial kinetic energy model to capture the seasonal 
cycle seen in the observations implies that on long time scales there might exist a balance between wind work on near-inertial motions and the loss of internal wave energy through breaking and ultimately, turbulent dissipation.

The use of equation 2.7 to represent the dissipation in the kinetic energy model carries an inherent assumption that all the work done by the near-inertial wind is radiated into the ocean interior as internal waves whose energy is, in turn, transferred to small vertical scales and eventually dissipated. Even though it is expected that some fraction of the work done by the wind on mixed layer inertial motions is dissipated locally in the mixed layer, it is difficult to determine what that fraction is. Skyllingstad et al. (2000) carried out an LES simulation with (spatially-uniform) inertially-resonant forcing which suggests that as much as half of the wind input into inertial motions is dissipated in the mixed layer. Thus for comparison, the kinetic energy model was run with the wind energy forcing term reduced by half. Reducing the wind forcing by half reduces the predicted time mean near-inertial kinetic energy level by a factor of 1.4. In addition, a third run was conducted in which the forcing was derived from the linear drag term of the PWP model (the term included in PWP to simulate radiation of energy out of the mixed layer into the stratified interior). Using the PWP drag forcing term results in a slight reduction of the predicted time mean near-inertial kinetic energy level by a factor of 1.1. All three of the model runs capture the seasonal cycle with maximum energy in winter. Comparison of the modeled seasonal cycle of near-inertial kinetic energy with the observations indicates that the root mean square error for all the model runs are within $70 \mathrm{Jm}^{-3}$ of each other, with the half wind forcing model run and the drag forcing model run having slightly smaller errors than the full model run.

However, the model has little to no skill capturing individual energetic events in the record; clearly propagation/advection effects are significant at shorter time scale. The largest observed depth-integrated near-inertial kinetic energy was recorded in the winter of 2001-2002, and corresponded with the presence of enhanced near-inertial kinetic energy at greater depths than in other years. Examination of the MMP 
observed temperature as well as satellite SST maps indicate that a warm core Gulf Stream ring was in the vicinity of Line $\mathrm{W}$ during the measured deep event. Warm core Gulf Stream rings can trap near-inertial internal waves, leading to increased energy levels (Kunze et al., 1995), and therefore could be responsible for the anomalous deep event.

The observation that the seasonal enhancement of near-inertial horizontal kinetic energy was most evident above 500 meters depth was used to estimate the horizontal extent of the surface forcing responsible for the near-inertial energy observed at the MMP mooring site, Table 2.2. Assuming a range of internal wave frequency values Table 2.2: Horizontal radius of influence (in $\mathrm{km}$ ) for various near-inertial frequencies

\begin{tabular}{ccccccc}
\hline \hline Frequency & $1.005 f$ & $1.01 f$ & $1.05 f$ & $1.1 f$ & $1.15 f$ & $1.2 f$ \\
\hline Angle with horizontal $\left(^{\circ}\right)$ & 0.11 & 0.15 & 0.35 & 0.50 & 0.62 & 0.72 \\
Horizontal radius of influence $(\mathrm{km})$ & 264 & 186 & 82 & 58 & 47 & 40 \\
\hline
\end{tabular}

ranging from $1.005 f$ to $1.2 f$, and estimating the mean $N$ value spanning 75 meters to 500 meters from all 4 MMP mooring deployments, the internal wave dispersion relation was used to calculate the angle of the internal wave group velocity vector with the horizontal. Applying the fact that the vertical extent of the enhanced energy was 500 meters yields a horizontal radius of influence which varies from $\sim 260$ kilometers to 40 kilometers. Any anomalously-strong near-inertial energy packet that is generated further away than the calculated radius of influence in any direction from the mooring site presumably dissipates before reaching the mooring site, or else it would be visible below 500 meters depth.

There are several ways this study could be improved upon. Increasing the sampling frequency of the MMP would be advantageous in two ways: (1) better resolve the near-inertial frequency motions, and (2) allow estimation of the horizontal energy flux. In turn, adding a coherent array of profilers would be useful for examining the energy flux divergence, and investigating the balances between the terms in the kinetic energy equation on an individual wind event basis. Measuring wind speed and 
surface velocity at the mooring site would allow for more accurate estimation of the wind energy input term. 


\section{Chapter 3}

\section{Observations of density and velocity using drifting EM-APEX floats during CLIMODE}

\subsection{Introduction}

Although mode waters, defined as large water masses characterized by weak vertical stratification, are widespread in the world oceans, the formation and maintenance mechanisms of these substantial water masses are still not well understood. Mode waters potentially have a large effect on climate conditions because they are annually re-exposed to the atmosphere and maintain "memory" of those encounters for years afterwards because of sea water's large heat capacity. For this study, we focus on the North Atlantic Subtropical Mode Water, or Eighteen Degree Water (EDW), which is found throughout the North Atlantic subtropical gyre south of the Gulf Stream at depth levels ranging from 200-500 meters. Marshall (2005) points out that a better understanding of the formation processes of EDW is important because it is an significant contributor to the Atlantic basin heat budget. It has also been shown that the strong wintertime heat flux over the Gulf Stream (thought to be important for EDW formation) influences the Atlantic storm track, which impacts the atmospheric 
meridional transport of heat (Hoskins and Valdes, 1990). Formation of North Atlantic Subtropical Mode Water is thought to take place during the wintertime passage of cold storms from the North American continent over the warm Gulf Stream, resulting in some of the largest air-sea heat fluxes in the world ocean. This heat flux leads to vigorous upper ocean convection, resulting in mixed layer depths as great as 500 meters. The newly formed mode water is then subducted as the ocean restratifies during spring, and advected throughout the subtropical gyre by the large scale wind driven circulation. Previous attempts to estimate the amount of EDW formed during a winter through direct (using floats) and indirect (using air-sea fluxes) methods yielded inconsistent results (Kwon and Riser, 2004a; Speer and Tziperman, 1992). It is thought that these discrepancies are due either to inaccurate estimates of the air-sea heat flux, or neglect of one or more important processes in the EDW volume budget. Specifically, it has been proposed that lateral eddy fluxes could play an important role in the redistribution of EDW. In order to address these issues, a large observational and modeling initiative known as CLIMODE (CLIvar Mode Water Dynamics Experiment) was undertaken. CLIMODE was designed to evaluate all the terms in the volume budget of EDW, and therefore diagnose the relative importance of those terms. Several different observational techniques were implemented as part of CLIMODE, including CTD sections, SeaSoar measurements, drifting profiling float measurements, surface drifters, moorings with meteorological and upper ocean instrumentation, and microstructure profiling. The variety of the resulting data set allows for investigation of upper ocean processes during EDW formation on a range of spatial and temporal scales.

The present work focuses on drifting profiling floats deployed in the southern flank of the Gulf Stream, which provide a quasi-Lagrangian view of the upper ocean during the wintertime formation of EDW. The combination of both surface meteorological forcing as well as upper ocean depth profiles of velocity, temperature, pressure, and salinity allow for direct evaluation of the upper ocean response to the large buoyancy and momentum fluxes associated with wintertime storm passage. These observations 
complement the SeaSoar, CTD, and microstructure data, which provide information about the evolving structure of the Gulf Stream. The goal of the drifting profiling float deployment was to evaluate departures of the upper ocean evolution from a onedimensional balance, with the residuals possibly being interpreted as the consequence of lateral eddy flux divergences.

\subsection{Deployment details}

Two Electromagnetic Autonomous Profiling Explorer (EM-APEX) profiling floats were deployed as part of the CLIMODE field program in February and March of 2007. The EM-APEX floats use an electromagnetic sensor to measure both horizontal ocean velocity components relative to a depth independent value, and also have a CTD which provides observations of conductivity, temperature, and pressure (Sanford et al., 2005). Both EM-APEX floats sampled from the surface to 550 meters depth for most of their deployments. The float 1636 was deployed for approximately one month while the float 1633 was deployed for approximately one week. At the end of each down-up profile pair, the floats surfaced to obtain a satellite GPS position fix and transmit the profile data to shore via satellite. On average, the EM-APEX floats took about one hour and twenty minutes to profile 550 meters (either up or down), and the floats generally spent about a half an hour on the surface between up and down profiles. There were a few instances in which the floats had difficulty acquiring a satellite fix; in these cases the floats drifted at the surface for longer time periods before resuming profiling activity. The floats were deployed in the southern core of the Gulf Stream; EM-APEX float 1633 stayed within the Gulf Stream for its week-long deployment, but EM-APEX float 1636 drifted south out of the jet during the second half of its month-long deployment, Figure 3-1. The EM-APEX floats measure a velocity profile which is relative to a depth-independent constant. That depth-independent constant is determined by forcing the time integration of the horizontal velocity from a down-then-up profile pair to equal the distance the float 

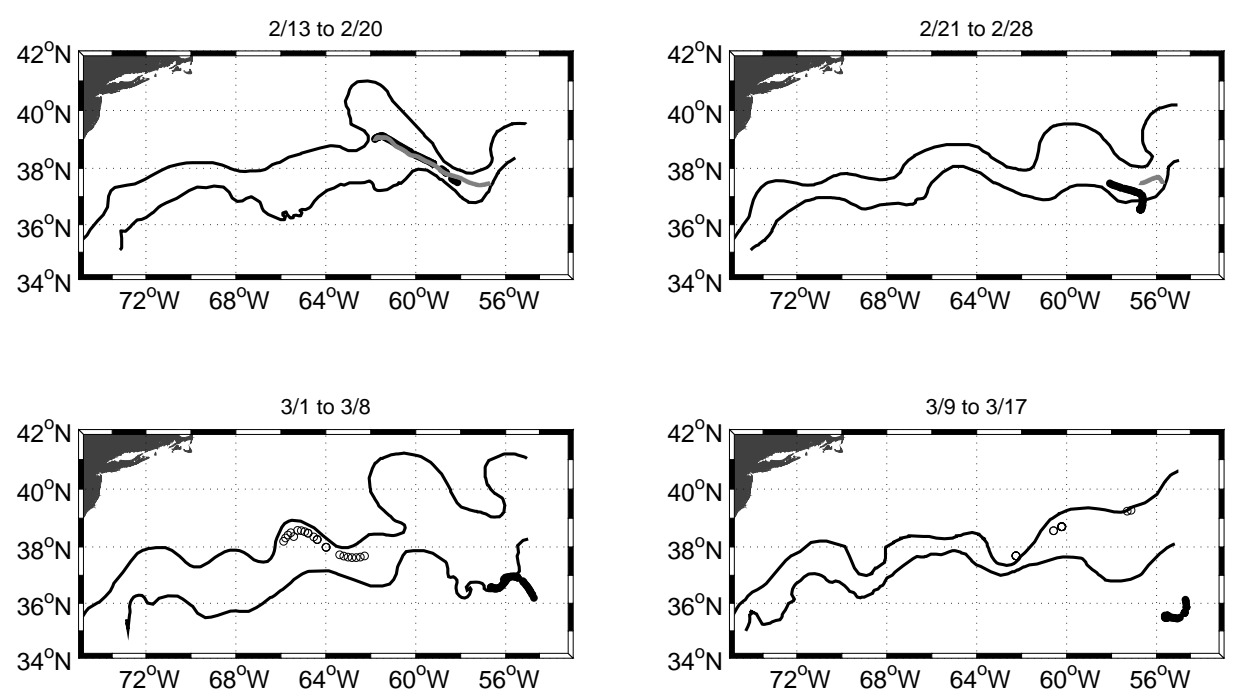

Figure 3-1: Positions of the EM-APEX 1636 float (black dots), EM-APEX 1633 float (black circles), and ASIS/FILIS (gray dots) with the north and south wall of the Gulf Stream (solid black lines). Each panel represents a one week interval, with the dates for each being noted.

traveled during that time, as determined from the GPS fixes the instrument acquires. This calculation carries the inherent assumption that the floats move with the local flow at each depth while profiling. The resultant absolute velocity estimates were then rotated into along-float-drift and across-float-drift directions based on the GPS positions.

In addition to the profiling floats, the Air Sea Interaction Spar buoy in combination with the tethered profiling Finescale Lagrangian Instrument System (ASIS/FILIS) was also deployed for a 9 day period in February of 2007. The ASIS/FILIS consisted of a "wire-crawling" profiling instrument measuring salinity, temperature, and pressure tethered to a drifting spar buoy which had a meteorological instrument package designed to allow for direct estimation of surface buoyancy fluxes. The distributed buoyancy at the top of the FILIS profiling wire was designed to minimize vertical heaving. The FILIS profiled to a depth of 450 meters, and the resulting data were binned to 2 dbar vertical resolution. One-way profiles took about an hour to com- 
plete, and there was generally a half hour wait between profiles. The ASIS/FILIS was deployed immediately before the EM-APEX float 1636, and the two drifted along a similar path for about a week before they diverged. However, the ASIS/FILIS traveled that path faster than the EM-APEX float 1636; the time their paths diverged, the ASIS/FILIS was about a hundred kilometers ahead of the float 1636. This spatial separation is probably a reflection of the fact that while the EM-APEX float follows the depth varying flow as it profiles vertically in this region of high shear, the ASIS/FILIS has a large surface buoy and therefore more closely tracks the high velocity Gulf Stream surface water. The ASIS/FILIS is also affected by wind and surface wave forcing which only act on the EM-APEX floats when they are on the surface getting a position fix.

\subsection{Results}

Initial evaluation of the EM-APEX and ASIS/FILIS deployments indicated that the goal of observing EDW during times of intense storm forcing was achieved. The time mean wind speed estimated from the EM-APEX deployment cruise shipboard meteorological data was $12 \mathrm{~ms}^{-1}$, with maximum speed reaching $27 \mathrm{~ms}^{-1}$, Figure 3-2. In addition to the strong winds, the shipboard observed air-sea temperature difference was quite large with a mean of $7^{\circ} \mathrm{C}$, and a maximum of $19{ }^{\circ} \mathrm{C}$. Both of the floats sampled water masses which can be classified as Eighteen Degree Water (EDW) according to the definition of Kwon and Riser (2004b), Figure 3-3. They define an EDW layer as being between 17 and $19{ }^{\circ} \mathrm{C}$ and also having a vertical temperature gradient less than $.006{ }^{\circ} \mathrm{Cm}^{-1}$. The EDW layer in the EM-APEX float 1633 record ranged from 100 to 200 meters thick, and was found between 200 and 400 meters depth. The EDW layer in both the EM-APEX float 1636 and FILIS records was thicker, reaching over 300 meters, and includes an apparent surface outcropping at the end of the deployment. In order to relate the strong surface buoyancy forcing to the upper ocean temperature evolution, a heat budget analysis of the EM-APEX 

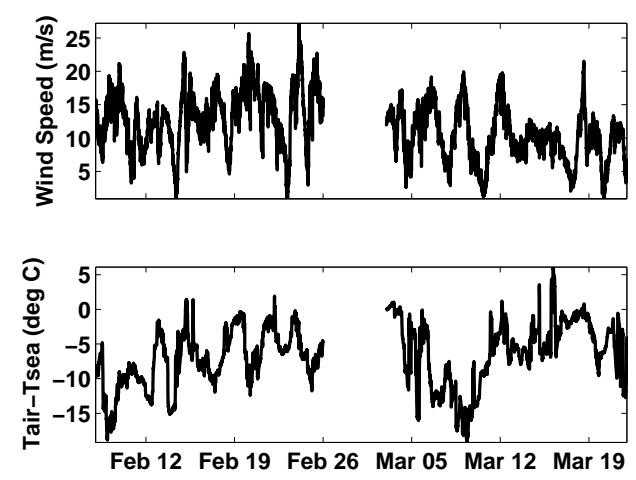

Figure 3-2: Shipboard wind speed (top panel) and air-sea temperature difference (bottom panel) for the CLIMODE EM-APEX float deployment cruises.

and ASIS/FILIS records was carried out. The details of this analysis can be found in Chapter 4 of this thesis.

The EM-APEX float velocity records are dominated by oscillations which appear to be near-inertial (i.e., with a period of approximately 20 hours), Figure 3-4. 

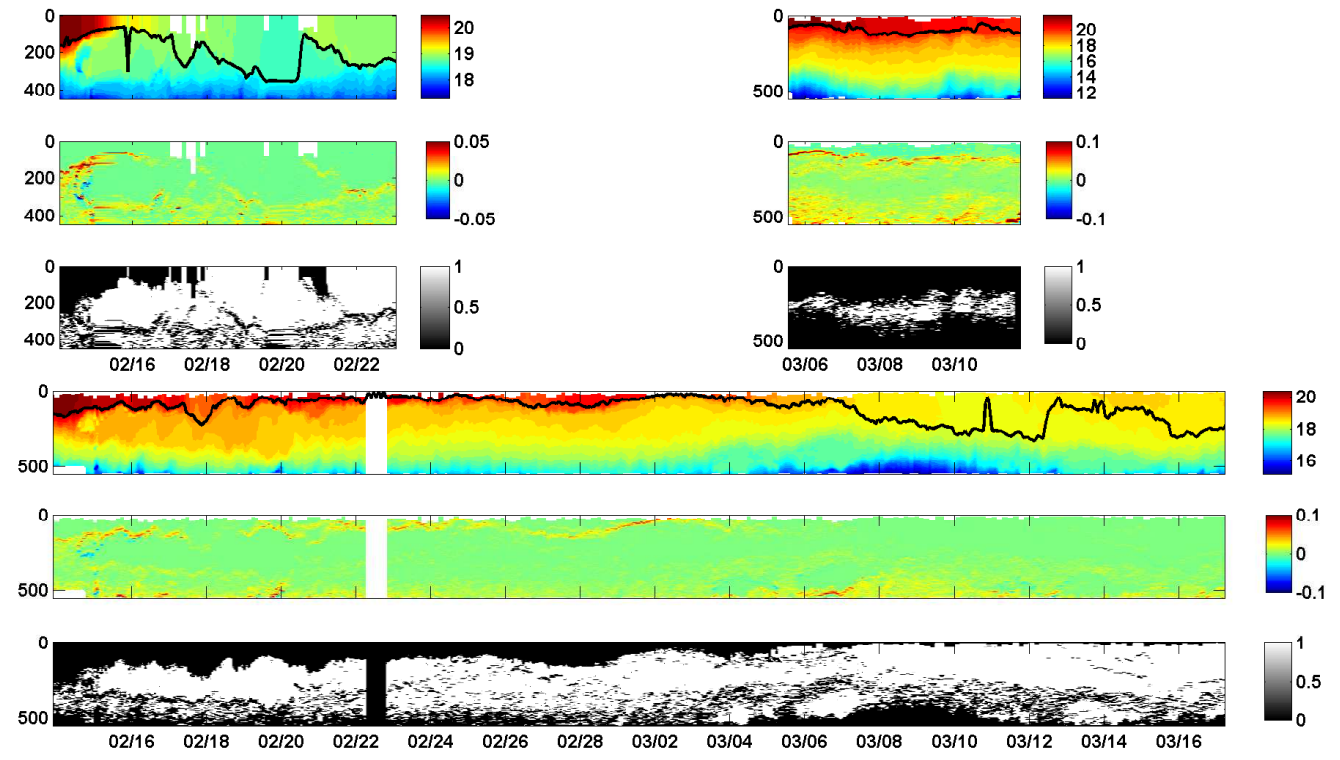

Figure 3-3: Temperature in ${ }^{\circ} \mathrm{C}$ (top panel) with mixed layer depth superimposed (black line), vertical temperature gradient in ${ }^{\circ} \mathrm{Cm}^{-1}$ (middle panel), and location of Eighteen Degree Water (white) for EM-APEX float 1633 (top right), float 1636 (bottom), and FILIS (top left). The mixed layer depth is defined here as the level where the temperature profile falls by $0.1^{\circ} \mathrm{C}$ from the sea surface temperature.
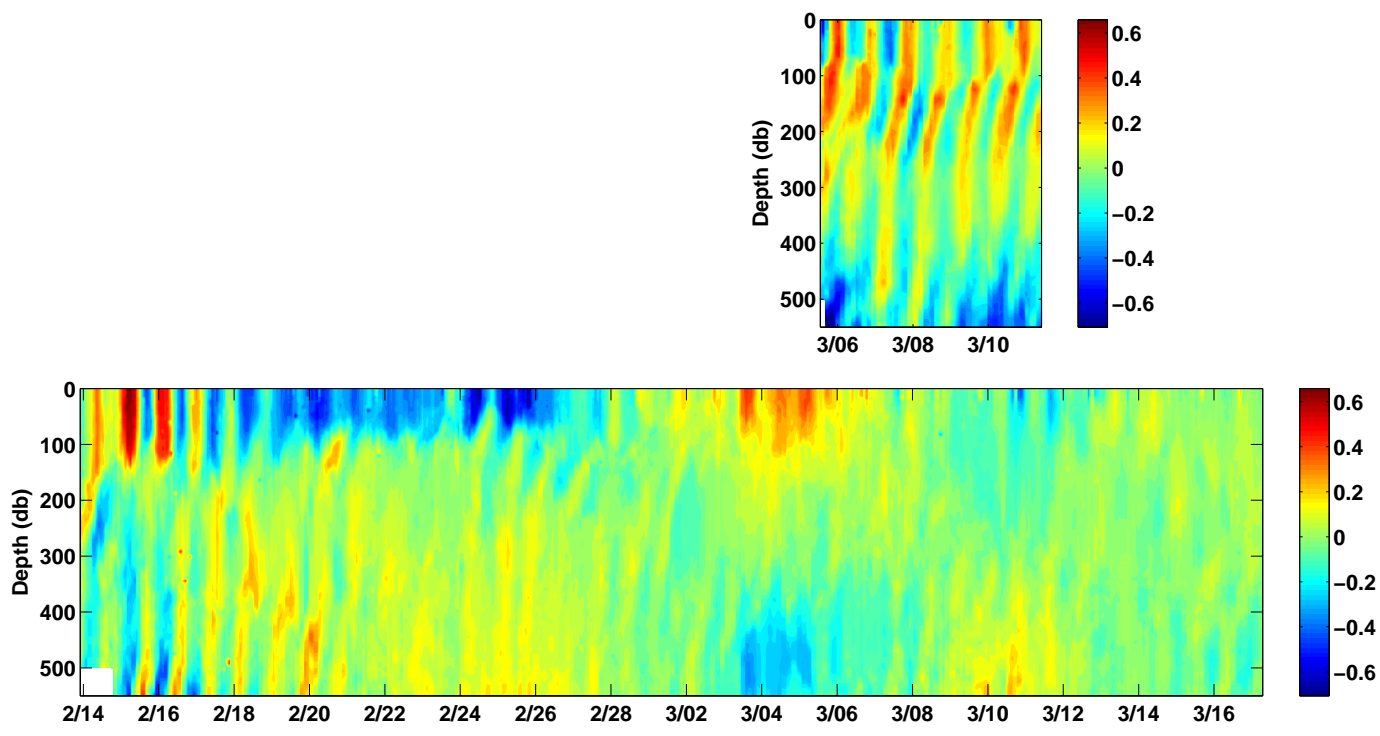

Figure 3-4: Observed zonal velocity with depth average removed for EM-APEX float 1633 (top) and float 1636 (bottom) in $\mathrm{ms}^{-1}$. 
The oscillations extend through the whole observed depth range (550 meters), have a greater magnitude towards the surface, and appear to be propagating vertically. In order to examine the frequency content, the velocity records were interpolated to a uniform time increment before spectral analyses were applied. Examination of the EM-APEX 1633 rotary frequency spectrum at 50 meters depth indicates that there is a large peak in the clockwise rotating in time energy near the local inertial frequency, Figure 3-5a. This peak is centered below the local inertial frequency for a resting ocean. Similarly, the EM-APEX 1636 rotary frequency spectrum displays a dominance of clockwise rotating energy with time during the first week of the deployment, with a peak occurring slightly below the local inertial frequency, Figure $3-5 b$. 

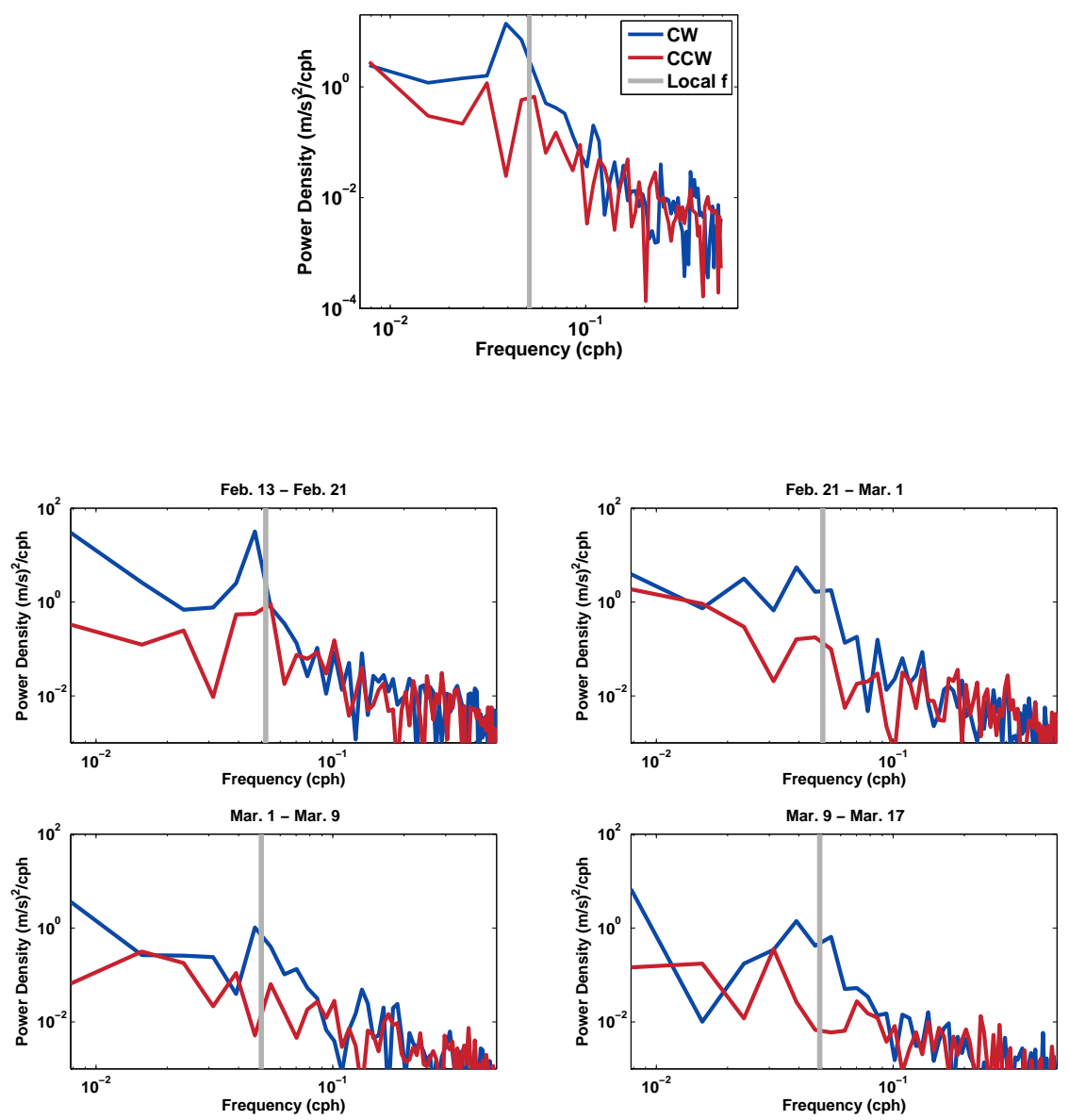

Figure 3-5: Rotary frequency spectra for EM-APEX float 1633 (top) and float 1636 (bottom) at 50 meters depth. The set of four lower panels are the spectra for each of the four weeks of the float deployment.

The shift in the near-inertial peak for float 1636 (week one) is slightly smaller than that observed for the 1633 float. There was not as notable a near-inertial peak for the remaining three weeks of the deployment. The rotary vertical wavenumber spectra for both floats reveal that motions with vertical wavelengths greater than 70 meters are dominated by clockwise rotating in depth energy, Figures 3-6a and 3-6b. 

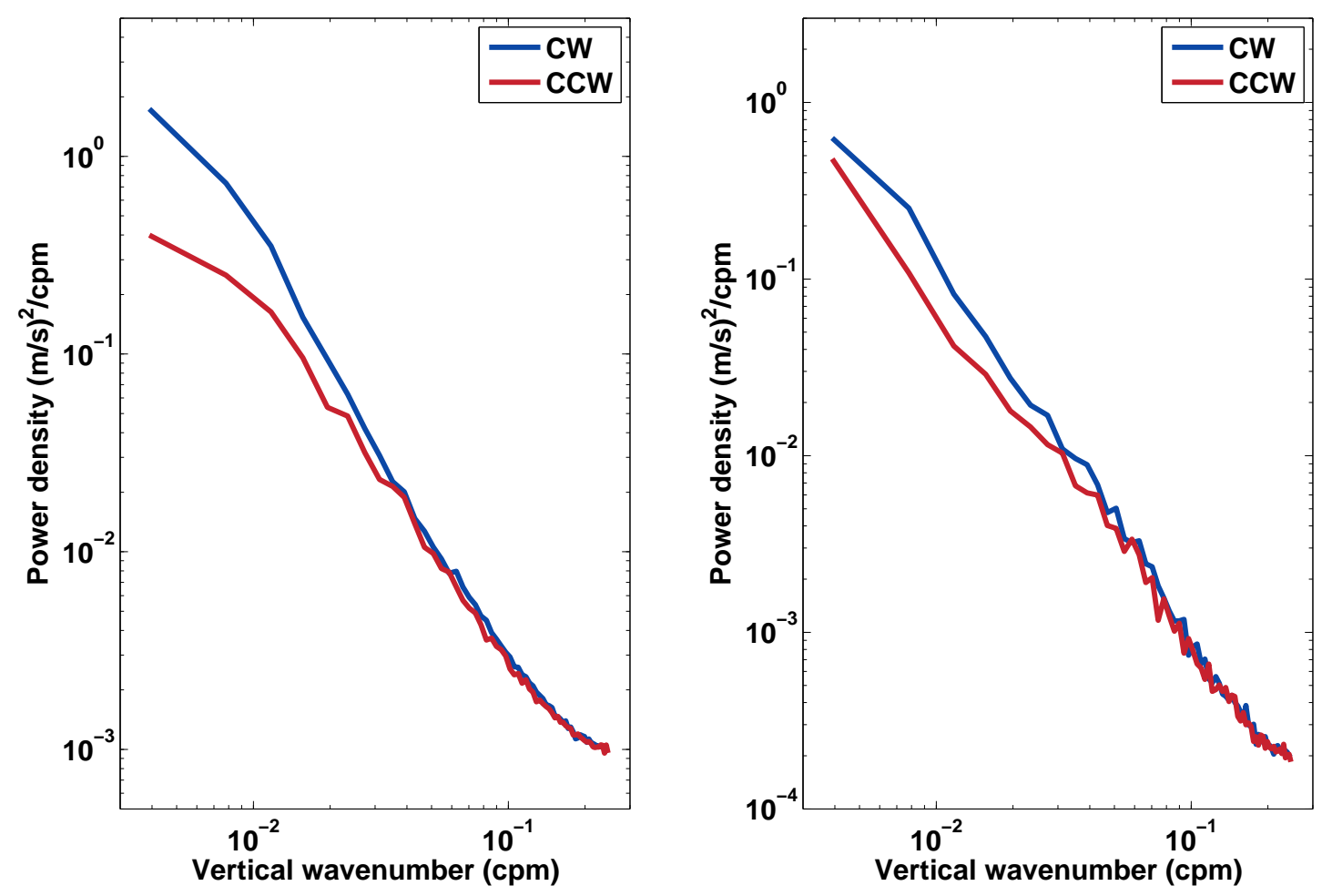

Figure 3-6: Rotary vertical wavenumber spectrum of the near-inertial velocity for EM-APEX float 1633 (left) and float 1636 (right) with a depth range of 200 to 500 meters.

The combination of clockwise rotation in time and depth (in the Northern Hemisphere) implies downward energy propagation, which is commonly observed in nearinertial motions, and is suggestive of surface (wind) forcing (Leaman and Sanford, 1975).

A more accurate estimate of the amplitude and frequency of the EM-APEX observed near-inertial motions is obtained using the extended complex demodulation technique (ECD) as described by Poulain (1990). The ECD method is simply a running least squares fit with sines and cosines to the observed velocity time series. The frequency of the fitted sinusoid is varied over a near-inertial frequency band and the dominant frequency is chosen as that frequency which minimizes the residual of the least square fit to the data. This analysis was repeated at each depth level, and yielded 

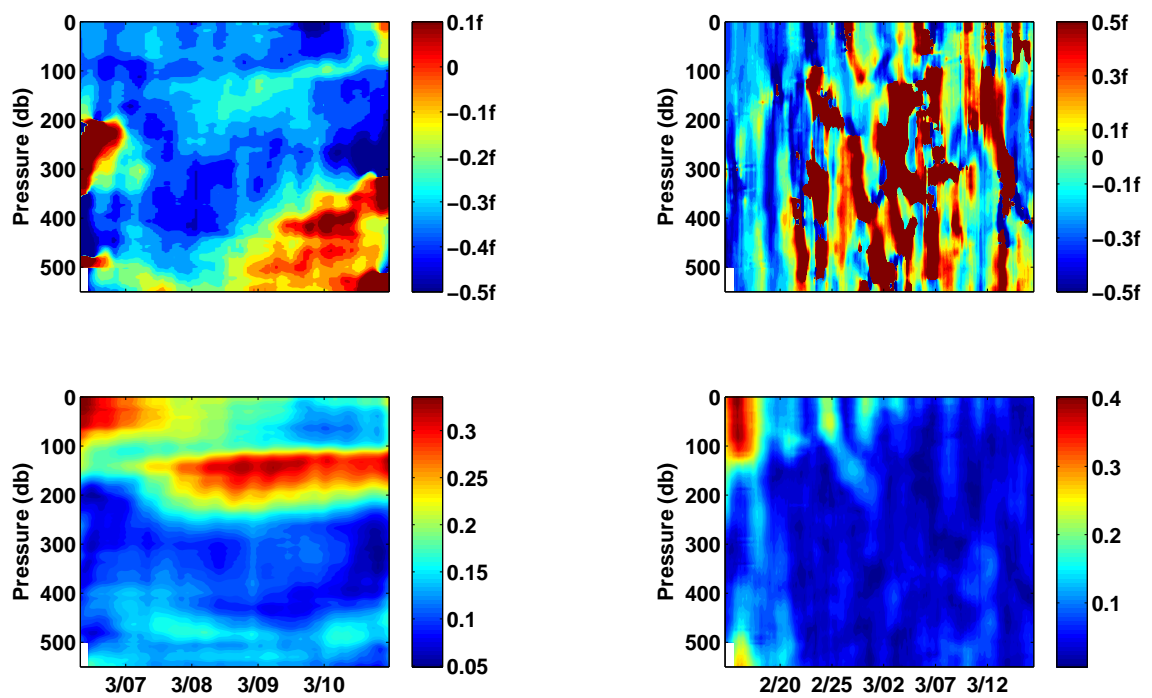

Figure 3-7: ECD frequency (top panels) and amplitude (bottom panels) for the EMAPEX 1633 float (left) and the EM-APEX 1636 float (right).

the temporal and depth evolution of the amplitude and frequency of the dominant observed oscillatory motions. The ECD results support the previous assertion that the near-inertial energy was surface intensified; near-inertial mixed layer amplitudes for both floats were as large as $40 \mathrm{cms}^{-1}$, Figure 3-7. The EM-APEX 1633 float sampled consistently energetic near-inertial motions for the duration of the weeklong deployment on the southern flank of the Gulf Stream. Similarly, the EM-APEX 1636 float had strong near-inertial motions in the Gulf Stream at the beginning of the deployment, but they became weaker as the float drifted southeast and out of the Gulf Stream high velocity core. The dominant frequency of the near-inertial motions was variable in space and time for both floats, Figure 3-7. The dominant frequency was depressed below the inertial frequency for a resting ocean from the surface down to 200 meters depth for the duration of the EM-APEX 1633 float deployment, and for the first week of the EM-APEX 1636 float deployment; the depressed near-inertial frequency was coincident with the times the floats were in the Gulf Stream jet.

The Price-Weller-Pinkel (PWP) one-dimensional mixed layer model was imple- 

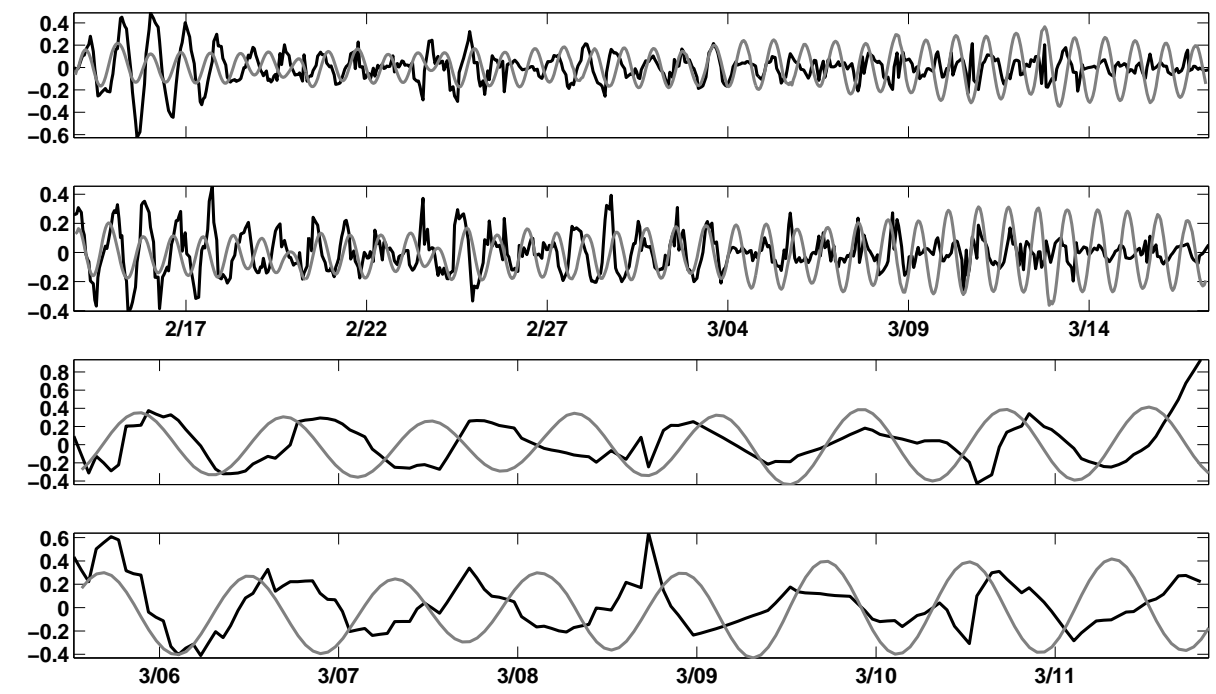

Figure 3-8: PWP model (gray line) and observed (black line) zonal (first and third panels) and meridional (second and fourth panels) velocities in $\mathrm{ms}^{-1}$ for EM-APEX float 1636 (top 2 panels), and EM-APEX float 1633 (bottom 2 panels).

mented for comparison with the EM-APEX float data. The PWP model consists of a slab-like mixed layer with constant velocity and density overlaying a transition layer. Parameterized mixing is invoked to alleviate critical bulk Richardson numbers at the base of the mixed layer, critical gradient Richardson numbers anywhere in the water column, and static instability at the base of the mixed layer. The wind and buoyancy forcing were estimated by applying the COARE algorithm to meteorological variables from the NCEP North American Mesoscale model interpolated to the float position (Fairall et al., 1996). For each run, the first float-observed density profiles and initial velocity profiles with time mean removed were used to initialize the model. The model was run with hourly temporal resolution and 2 meter vertical resolution. Comparison of the observed mixed layer velocity components and the model output shows that the PWP model captures the basic order of magnitude of the near-inertial oscillations, Figure 3-8. This suggests that the upper ocean wind forcing during the deployments was of sufficient strength to force the observed energetic near-inertial 
motions. The depression of the near-inertial frequency below that for a resting ocean can be seen in the phase mismatch between the model and observed currents.

\subsection{Discussion}

Freely propagating oceanic internal waves have frequencies ranging from the local inertial frequency to the buoyancy frequency. Therefore, motions with a frequency below the lower bound frequency, as we have observed here, may have interesting propagation characteristics. Several authors have recorded similar observations from a variety of locations, including the New England Shelf (Shearman, 2005), the Japan/East Sea (Park and Watts, 2005), and the tropical Pacific Ocean (Poulain et al., 1992). There are two possible explanations for the observed depression of the frequency below the value of $f$ for a resting ocean. The first is Doppler shifting, which is an artifact of the measurement method that can occur when inertial oscillations are advected past the measurement system and appear to be at a different frequency than they would appear to a Lagrangian observer. McNeil et al. (1999) explain an observation of depressed inertial frequency from a mooring near Bermuda with Doppler shifting, using the fact that Doppler shifting is dependent on both the velocity of the flow past the measurement device and the horizontal wavelength of the inertial wavefield. Because the EM-APEX floats are drifting with the flow at each depth level, the relative velocity past the floats (due to the fact that the floats are profiling vertically in the presence of large vertical shear) is small $\left(\sim 10 \mathrm{cms}^{-1}\right)$. The horizontal wavelength required to produce the observed shift in frequency is around 10 kilometers. The horizontal length scale of near-inertial motions is set by atmospheric forcing (hundreds of kilometers), though it can be influenced by mesoscale flow features, which may introduce scales of tens of kilometers. Therefore, it seems unlikely that the horizontal wavelength of the flow field would have been small enough to produce the observed shift in frequency, and so Doppler shifting is eliminated as a potential explanation.

The second possibility is the presence of a strong negative relative vorticity signal. 
There is a fair amount of observational precedence for this phenomenon; data from frontal/jet systems as well as eddies with both positive and negative vorticity have been used to demonstrate the influence of a strong relative vorticity signal on nearinertial oscillations (Pingree and Cann, 1991; Poulain, 1990). Perkins (1976) shows that the effective inertial frequency may be written in terms of the relative vorticity as follows:

$$
f_{\text {eff }}^{2}=f_{0}\left(f_{0}+\zeta\right)=f_{0}\left(f_{0}+\left[\frac{\partial V}{\partial x}-\frac{\partial U}{\partial y}\right]\right),
$$

and uses this relation to account for a mooring observation of depressed inertial frequency using relative vorticity estimates from local synoptic surveys. Similarly, we can test this explanation by comparing the observed effective frequency of the oscillations with that predicted using the observed relative vorticity about the floats. There were not data available to do this analysis for the EM-APEX 1636 float. However, for the EM-APEX 1633 float, several SeaSoar sections were obtained across the float drift track which may be used to estimate relative vorticity, and in turn the effective $f$, Figure 3-9. The SeaSoar provides one component of the velocity; by assuming that the other derivative (the along stream derivative of the across stream flow) and curvature are small, the effective $f$ can be estimated. The depression of the inertial frequency in the mixed layer shows good agreement with the observed relative vorticity signal for both SeaSoar sections; the predicted and observed depression for the first section were both $-0.4 f$, while the predicted depression for the second section was $-0.2 f$, and the observed depression was - $-0.3 f$. This indicates that the EM-APEX floats captured evidence of the response of the near-inertial oscillations to the mesoscale flow field.

Interesting dynamical consequences of the propagation of near-inertial motions through background mesoscale flow fields have been highlighted in a series of modeling and observational studies. D'Asaro et al. (1995) reported a discrepancy between the analytical prediction of mixed layer near-inertial decay time and observations during the Ocean Storms experiment. Subsequent model work seemed to suggest that the smaller horizontal scales imparted to the near-inertial oscillations by the background flow field leads to a more rapid propagation of near-inertial energy into the ocean 


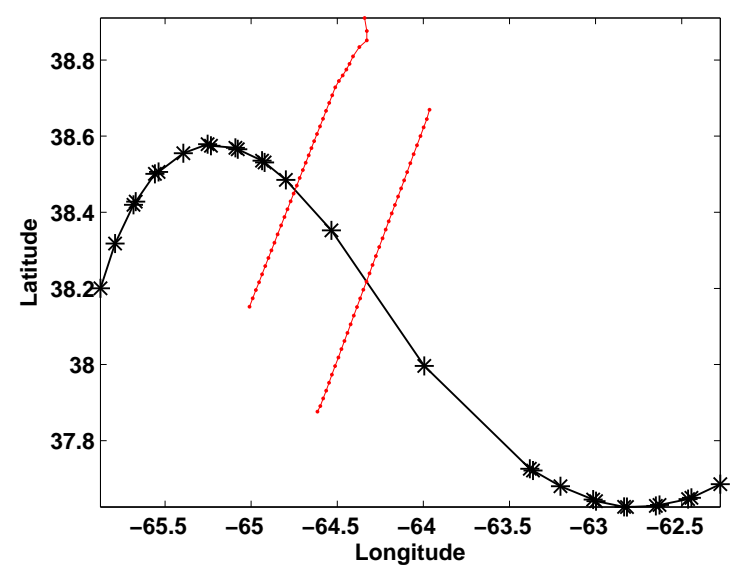

Figure 3-9: EM-APEX float 1633 drift track (black dots) with shipboard SeaSoar sections (red dots) used for relative vorticity calculation. The float track shown covers March 5th 2007, to March 8th 2007, while the SeaSoar sections were taken on March 7th and March 8th, 2007.

interior (Balmforth and Young, 1999). Mooring observations support the notion that the decay time of mixed layer near-inertial motions is dependent on the gradient of the relative vorticity (Meurs, 1998). Thus, uniform wind forcing of near-inertial motions in a region with relative vorticity can rapidly lead to spatial variations in mixed layer properties.

Motions which are forced in negative vorticity regions and have an effective frequency below the local inertial frequency cannot freely propagate out of those regions (Kunze and Sanford, 1984). Thus, near-inertial energy which has radiated vertically out of the mixed layer in negative vorticity regions is subject to the trapping of near-inertial waves. This assertion has been corroborated by modeling work which demonstrates that wind-forced near-inertial energy builds up in mesoscale negative vorticity structures (Danioux et al., 2008; Lee and Niiler, 1998). The trapped nearinertial internal waves are thought to grow in amplitude until the energy is dissipated 
through shear instability processes (Kunze et al., 1995), potentially leading to localized homogenization of scalar properties.

As noted above, formation of Eighteen Degree Water is thought to occur on the south side of the Gulf Stream, which is the negative relative vorticity side of the jet. Interaction of the Gulf Stream jet with wind forced near-inertial oscillations can lead to spatial variations in the mixed layer, which could have an effect on the mixed layer response to the strong buoyancy forcing thought to be responsible for EDW formation. In addition to this, near-inertial wave trapping in the negative vorticity core of the Gulf Stream could produce enhanced mixing in the ocean interior in the EDW formation region. These potential impacts on EDW formation cannot be assessed with the EM-APEX float data, which provides only a transitory view of the formation process. Therefore, a three dimensional primitive equation ocean model was utilized to investigate these issues. The results from that analysis are reported in Chapter 5 of this thesis. 


\section{Chapter 4}

\section{A wintertime quasi-Lagrangian heat budget in the Gulf Stream region}

\subsection{Introduction}

As described in the previous chapter, one of the main goals of the CLIMODE (CLIVAR Mode Water Dynamics Experiment) field program is to evaluate the importance of previously neglected processes during the formation of North Atlantic Subtropical Mode Water, or Eighteen Degree Water (EDW). Because the surface heat fluxes between the warm Gulf Stream waters and the cold wintertime air masses in the North Atlantic are some of the largest in the world ocean, and therefore generate rigorous convection in the upper ocean, it has been long been hypothesized that the formation of the weakly stratified mode water is dominated by air-sea buoyancy fluxes. However, there is a mismatch between an air-sea flux based prediction of the volume of mode water formed and the actual amount of mode water formed (Kwon and Riser, 2004a), suggesting that either this hypothesis is not entirely accurate or the available data was insufficient to perform the calculation. There is an active eddy field in the EDW formation region due to Gulf Stream instabilities, and therefore it has been 
suggested that lateral eddy fluxes could potentially be the missing process. It is also possible that the use of poor resolution air-sea interaction data led to an inaccurate estimate of the surface buoyancy flux. The EM-APEX drifting profiling floats and ASIS-FILIS drifting system, which were deployed as part of CLIMODE in the EDW formation region during winter, provide an opportunity to evaluate the Lagrangian upper ocean response during EDW formation. Other CLIMODE instrumentation provide data needed to estimate high resolution surface buoyancy flux during the float deployments. In order to quantify the upper ocean thermal evolution during EDW formation, these float data are used here in a heat budget analysis.

Heat budgets are a common tool in physical oceanography, and have been used for a variety of purposes, from studying the influences on SST in the tropical oceans (Cronin and McPhaden, 1997) to estimating the vertical heat flux during convection in the Labrador Sea (Lavender et al., 2002). There are numerous examples of both modeling and observational heat budget studies, and some that combine the two methods. Modeling studies tend to examine the heat budget in a large control volume, while some observational heat budgets take advantage of arrays of moorings to do the same. However, the most common observational studies use data from a single mooring or drifting instrument to follow the time evolution of the depth integrated heat content. Evaluating the terms in the heat equation over long time periods can provide insight into the role of the ocean in climate change, while shorter timescale heat budgets are useful for tracking the upper ocean response to wind and buoyancy forcing events.

Previous model heat budget studies in the Gulf Stream region have suggested that advection can be an important contributor to changes in heat storage on large spatial and long time scales (Kelly and Qui, 1995; Dong and Kelly, 2004). However, Xue et al. (1994) examined moored observations of the response of the Gulf Stream to the passage of a wintertime storm characterized by strong cooling and concluded that changes in heat storage are dominated by the surface heat flux. This study focuses on observations from drifting profiling instruments, which are quasi-Lagrangian, and 
consequently reduce, but as we will see, don't eliminate the effects of horizontal advection. It was hoped that the heat budget following these free-drifting instruments would be largely one-dimensional; that is that changes in upper ocean heat storage would be closely related to the surface heat flux, and that the evolution of the mixed layer depth could be predicted from the known surface forcing.

Heat budget analysis of observations taken from three drifting instruments deployed during CLIMODE in the winter of 2007 are examined below. Surface heat fluxes are derived from a mesoscale meteorological model and verified with the CLIMODE direct air-sea heat flux measurements. In spite of the large wind and buoyancy forcing and the quasi-Lagrangian nature of the floats, the heat budget is found to be dominated by the advective terms. In addition, the mixed layer depth evolution following the drifting instruments does not correspond to the observed surface forcing. The advection is shown to be a result of the displacement of mixed layer water parcels relative to the drifting instruments due to the large vertical current shear of the Gulf Stream.

\subsection{Data and methods}

Details of the float deployment can be found in Chapter 3 of this thesis.

\subsubsection{Heat budget equation}

There are two ways in which observational studies have implemented depth-integrated heat budgets: using a fixed integration depth, or using a time varying depth such as an isotherm depth or the mixed layer base. Using a fixed integration depth is usually more convenient, but changes in the resulting heat content tend to be dominated by vertical displacements of the thermocline (due to the passage of internal waves and/or eddies), and so are not particularly useful for evaluating the effects of surface forcing (Stevenson and Niiler, 1983). Therefore, for the purposes of this study, the depth integration of the heat budget is carried out using a time varying integration 
depth, $h(t)$. The derivation of the heat equation integrated to a time varying depth as introduced by Stevenson and Niiler (1983) begins with the heat and mass conservation equations:

$$
\begin{gathered}
\rho c_{p} \frac{D T}{D t}=\frac{\partial Q}{\partial z} \\
\frac{\partial u}{\partial x}+\frac{\partial v}{\partial y}+\frac{\partial w}{\partial z}=0
\end{gathered}
$$

The coordinate system is rotated such that the $\mathrm{x}$ direction now represents the along float drift direction, and the y direction represents the across float drift direction. The heat equation becomes

$$
\rho c_{p}\left[\frac{d T}{d t}+\mathbf{u} \cdot \nabla T+w \frac{\partial T}{\partial z}\right]=\frac{\partial Q}{\partial z},
$$

where $\mathbf{u}$ represents the horizontal ocean velocity relative to the float, and $\frac{d T}{d t}$ represents the temporal derivative of the temperature field observed by the floats.

Next, the temperature and horizontal velocity are decomposed as $\left(T=T_{a}+\hat{T}\right)$, $\left(u=u_{a}+\hat{u}\right)$ and $\left(v=v_{a}+\hat{v}\right) . T_{a}, u_{a}$ and $v_{a}$ represent the vertical mean of temperature, zonal, and meridional velocity between the surface and depth $h$, and $\hat{T}, \hat{u}$, and $\hat{v}$ represent the departures from those means. Applying this decomposition and integrating in depth yields:

$$
\begin{gathered}
\rho c_{p} \int_{-h}^{0}\left[\frac{d T_{a}}{d t}+\frac{d \hat{T}}{d t}\right] d z+ \\
\rho c_{p} \int_{-h}^{0}\left[\left(u_{a}+\hat{u}\right) \frac{\partial\left(T_{a}+\hat{T}\right)}{\partial x}+\left(v_{a}+\hat{v}\right) \frac{\partial\left(T_{a}+\hat{T}\right)}{\partial y}+w \frac{\partial\left(T_{a}+\hat{T}\right)}{\partial z}\right] d z \\
=\int_{-h}^{0} \frac{\partial Q}{\partial z} d z .
\end{gathered}
$$

The first integral on the LHS of equation (4) simplifies as:

$$
\int_{-h}^{0}\left[\frac{d T_{a}}{d t}+\frac{d \hat{T}}{d t}\right] d z=h \frac{d T_{a}}{d t}+\int_{-h}^{0} \frac{d \hat{T}}{d t} d z
$$




$$
\begin{aligned}
& =h \frac{d T_{a}}{d t}+\frac{d}{d t} \int_{-h}^{0} \hat{T} d z-\hat{T}_{-h} \frac{d h}{d t} \\
& =h \frac{d T_{a}}{d t}-\hat{T}_{-h} \frac{d h}{d t} .
\end{aligned}
$$

The along float drift advection terms in equation (4) can be written as:

$$
\begin{aligned}
\int_{-h}^{0}\left[\left(u_{a}+\hat{u}\right) \frac{\partial\left(T_{a}+\hat{T}\right)}{\partial x}\right] d z & =\int_{-h}^{0}\left[u_{a} \frac{\partial T_{a}}{\partial x}+u_{a} \frac{\partial \hat{T}}{\partial x}+\hat{u} \frac{\partial \hat{T}}{\partial x}\right] d z \\
& =h u_{a} \frac{\partial T_{a}}{\partial x}+\int_{-h}^{0}\left[\left(u_{a}+\hat{u}\right) \frac{\partial \hat{T}}{\partial x}\right] d z
\end{aligned}
$$

Similarly, the across float drift advection terms are:

$$
\int_{-h}^{0}\left[\left(v_{a}+\hat{v}\right) \frac{\partial\left(T_{a}+\hat{T}\right)}{\partial y}\right] d z=h v_{a} \frac{\partial T_{a}}{\partial y}+\int_{-h}^{0}\left[\left(v_{a}+\hat{v}\right) \frac{\partial \hat{T}}{\partial y}\right] d z
$$

Rewriting the vertical advection term with the continuity equation yields:

$$
\begin{aligned}
\int_{-h}^{0} w \frac{\partial\left(T_{a}+\hat{T}\right)}{\partial z} d z & =\int_{-h}^{0}\left[\frac{\partial}{\partial z} w\left(T_{a}+\hat{T}\right)-\left(T_{a}+\hat{T}\right) \frac{\partial w}{\partial z}\right] d z \\
& =-w_{-h} \hat{T}_{-h} \\
& +\int_{-h}^{0}\left[\hat{T}\left(\frac{\partial}{\partial x}\left(u_{a}+\hat{u}\right)\right)+\hat{T}\left(\frac{\partial}{\partial y}\left(v_{a}+\hat{v}\right)\right)\right] d z
\end{aligned}
$$

Combining all the advection terms produces:

$$
\begin{aligned}
\int_{-h}^{0}\left[\left(u_{a}+\hat{u}\right) \frac{\partial\left(T_{a}+\hat{T}\right)}{\partial x}+\left(v_{a}+\hat{v}\right) \frac{\partial\left(T_{a}+\hat{T}\right)}{\partial y}+w \frac{\partial\left(T_{a}+\hat{T}\right)}{\partial z}\right] d z \\
=h u_{a} \frac{\partial T_{a}}{\partial x}+h u_{a} \frac{\partial T_{a}}{\partial y} \\
+\int_{-h}^{0}\left(\frac{\partial u_{a} \hat{T}}{\partial x}+\frac{\partial \hat{u} \hat{T}}{\partial x}+\frac{\partial v_{a} \hat{T}}{\partial y}+\frac{\partial \hat{v} \hat{T}}{\partial y}\right) d z+w_{-h} \hat{T}_{-h}
\end{aligned}
$$




$$
=h \mathbf{u}_{\mathbf{a}} \cdot \nabla T_{a}+\nabla \cdot \int_{-h}^{0} \hat{\mathbf{u}} \hat{T} d z-\hat{T}_{-h}\left(\mathbf{u}_{-\mathbf{h}} \cdot \nabla h-w_{-h}\right) .
$$

Combining all the terms results in the heat budget equation:

$\rho c_{p}[\underbrace{h \frac{d T_{a}}{d t}}_{I}+\underbrace{h \mathbf{u}_{\mathbf{a}} \cdot \nabla T_{a}}_{I I}+\underbrace{\nabla \cdot\left(\int_{-h}^{0} \hat{\mathbf{u}} \hat{T} d z\right)}_{I I I}-\underbrace{\hat{T}_{-h}\left(\frac{d h}{d t}+\mathbf{u}_{-\mathbf{h}} \cdot \nabla h+w_{-h}\right)}_{I V}]=\underbrace{\left(Q_{o}-Q_{-h}\right)}_{V}$.

where $Q_{o}$ and $Q_{-h}$ are the heat fluxes at the surface and base of the integrated layer, respectively, and $\rho c_{p}$ is the volumetric heat capacity of seawater. This equation shows that the local heat storage in the integration layer $(I)$ can be changed by relative advection of the horizontal gradient of the depth mean temperature by the depth mean relative flow $(I I)$, the divergence of the baroclinic velocity and temperature profile covariance $(I I I)$, entrainment at the base of the layer (due to either the movement of the integration depth in time or both vertical and horizontal advection) $(I V)$, and vertical turbulent heat fluxes at the surface and base of the integration layer $(V)$.

The choice of lower boundary for the heat budget is dependent on the observational situation. Generally, isotherm heat budgets use lower boundary isotherms which are in the thermocline where the entrainment and vertical turbulent heat fluxes are negligible. These simplifications give

$$
\rho c_{p}\left[h \frac{d T_{a}}{d t}+h v_{a} \frac{\partial T_{a}}{\partial y}+\nabla \cdot\left(\int_{-h}^{0} \hat{\mathbf{u}} \hat{T} d z\right)\right]=Q_{o}
$$

which shows that an isotherm heat budget is useful for determining if the evolution of the observed heat storage is dominated by the surface heat flux or horizontal advection. As it is usually difficult to determine vertical velocity and turbulent heat flux from the available oceanographic measurements (such as moored current meter and temperature recorders), this approach is advantageous. However, it is important to choose an isotherm which is as shallow as possible in order to ensure that the derived changes in heat content represent surface as opposed to thermocline processes 
(Feng et al., 1998). A common problem with isotherm heat budgets is determining the depth-dependent horizontal advection of temperature into the integration region, which is usually difficult to estimate from available data. Therefore an advantage of carrying out a mixed layer heat budget is being able to neglect the divergence of the baroclinic temperature and velocity covariance (which one may argue is negligible due to the weak depth dependence of temperature and velocity in the mixed layer), giving

$$
\rho c_{p}\left[h \frac{d T_{a}}{d t}+h v_{a} \frac{\partial T_{a}}{\partial y}-\hat{T}_{-h}\left(\frac{d h}{d t}+u_{-h} \frac{\partial h}{\partial x}+v_{-h} \frac{\partial h}{\partial y}+w_{-h}\right)\right]=Q_{o}-Q_{-h}
$$

Of course the entrainment and vertical turbulent heat flux at the base of the mixed layer are not expected to be small, so a mixed layer heat budget can be useful for examining how the contribution of mixing to the evolution of the heat storage compares to that of depth mean advection and the surface heat flux. For this study, it was found that errors in an isotherm heat budget were unacceptably large, and so the following chapter will describe a mixed layer heat budget analysis. Methods used to estimate each term in the heat budget will be discussed in the next section.

\subsubsection{Determination of one-dimensional heat budget terms}

The mixed layer depth (MLD) is defined here as the level where the temperature profile falls by $0.1{ }^{\circ} \mathrm{C}$ from the sea surface temperature. The results presented here are not sensitive to the MLD definition; changing the MLD criterion to 0.2 or even $0.5^{\circ} \mathrm{C}$ does not qualitatively change the one-dimensional heat budget results for any of the instrument systems. The time mean MLDs for the EM-APEX 1633 and 1636, and FILIS deployments were 98 meters, 110 meters, and 200 meters, respectively.

The temporal derivative of the depth mean temperature multiplied by the mixed layer depth, or the heat storage rate, is estimated using the temperature profiles recorded by the EM-APEX floats and the FILIS. The time derivative of the depth mean temperature is derived using a first order centered difference scheme. 
The surface heat flux is usually one of the largest terms in the heat budget, and consequently it is desirable to determine this term with as much accuracy as possible. The surface heat flux is the sum of the turbulent heat fluxes (sensible and latent) and the radiative heat fluxes (shortwave and longwave). As part of the CLIMODE initiative, the NCEP NAM (North American Mesoscale) meteorological model (which is implemented by the National Weather Service), which is a data assimilation model, was run at quarter-degree spatial resolution and three hour temporal resolution. Meteorological output from the NAM model (interpolated in space and time to follow each of the drifting instruments) along with the float SST are used as input for the COARE algorithm (a frequently used bulk air-sea algorithm that has been validated over a range of forcing regimes (Fairall et al., 2003)), to provide estimates of the sensible and latent heat fluxes at the drifting instrument locations for use in the heat budget. Using this method to determine the turbulent heat fluxes at the ship locations during the CLIMODE cruises provides means for comparison with the shipboard estimates of the direct covariance fluxes. Because there are no radiometer data available, the radiative heat fluxes for the CLIMODE heat budget were obtained directly from the output of the NAM model.

For the one dimensional heat budget calculation, the heat storage rate and the total surface heat flux were smoothed with a 48 hour running averaging filter. Error bar estimation on all the terms in the heat budget was carried out using either the propagation of errors method, or comparison with other data sources, and is described in detail in Appendix C. The uncertainty in the total surface heat flux was found to be $\pm 150 \mathrm{Wm}^{-2}$, and the time mean uncertainty in the FILIS, EM-APEX 1636, and EM-APEX 1633 heat storage rates is $\pm 600 \mathrm{Wm}^{-2}, \pm 140 \mathrm{Wm}^{-2}$, and $\pm 170 \mathrm{Wm}^{-2}$ respectively. 


\subsection{One dimensional heat budget results}

\subsubsection{Surface forcing}

Throughout this paper, the sign convention is that negative(positive) surface heat fluxes are cooling(warming) the ocean. Sensible, latent, and longwave surface heat fluxes acted to cool the ocean for the duration of the CLIMODE drifting instrument deployments, Figure 4-1. Of those three surface heat flux components, the latent flux contributed the most, with the sensible flux generally being the next largest. The latent and at times even the sensible heat fluxes alone were larger in magnitude than the peak shortwave heat flux. As expected, the net surface heat flux values were quite large in comparison with other oceanic regions; the most extreme hourly estimated net heat flux for EM-APEX 1636, EM-APEX 1633, and the FILIS system were -997 $W m^{-2},-1298 W^{-2}$, and $-830 W^{-2}$ respectively. The wind stress series following

all three floats is marked by strong episodic peaks of short duration (a few days), which is indicative of the passage of wintertime storms. The EM-APEX 1636 and the FILIS experienced large wind stresses greater then $1 \mathrm{Nm}^{-2}$, while the EM-APEX float 1633 experienced somewhat weaker wind stresses with a maximum of about $0.6 \mathrm{Nm}^{-2}$. The wind stress events at times seems to be associated with large negative peaks in surface heat flux (for example, as can be seen around February 20th in both the EM-APEX float 1636 and the ASIS/FILIS records). However, this doesn't seem to be always true; although there is a strong wind stress peak on February 15th in the ASIS/FILIS record, the total surface heat flux stays relatively steady (but negative) over that time. Closer examination of the ASIS/FILIS temperature time series reveals that the air temperature was relatively close to the sea surface temperature during this wind event, and analysis of the wind direction shows that the winds were coming from the southeast. Therefore, although these surface forcing records reflect the passage of wintertime storms, not all of these storms are carrying cold air which will result in large surface heat loss, and ultimately EDW formation. 

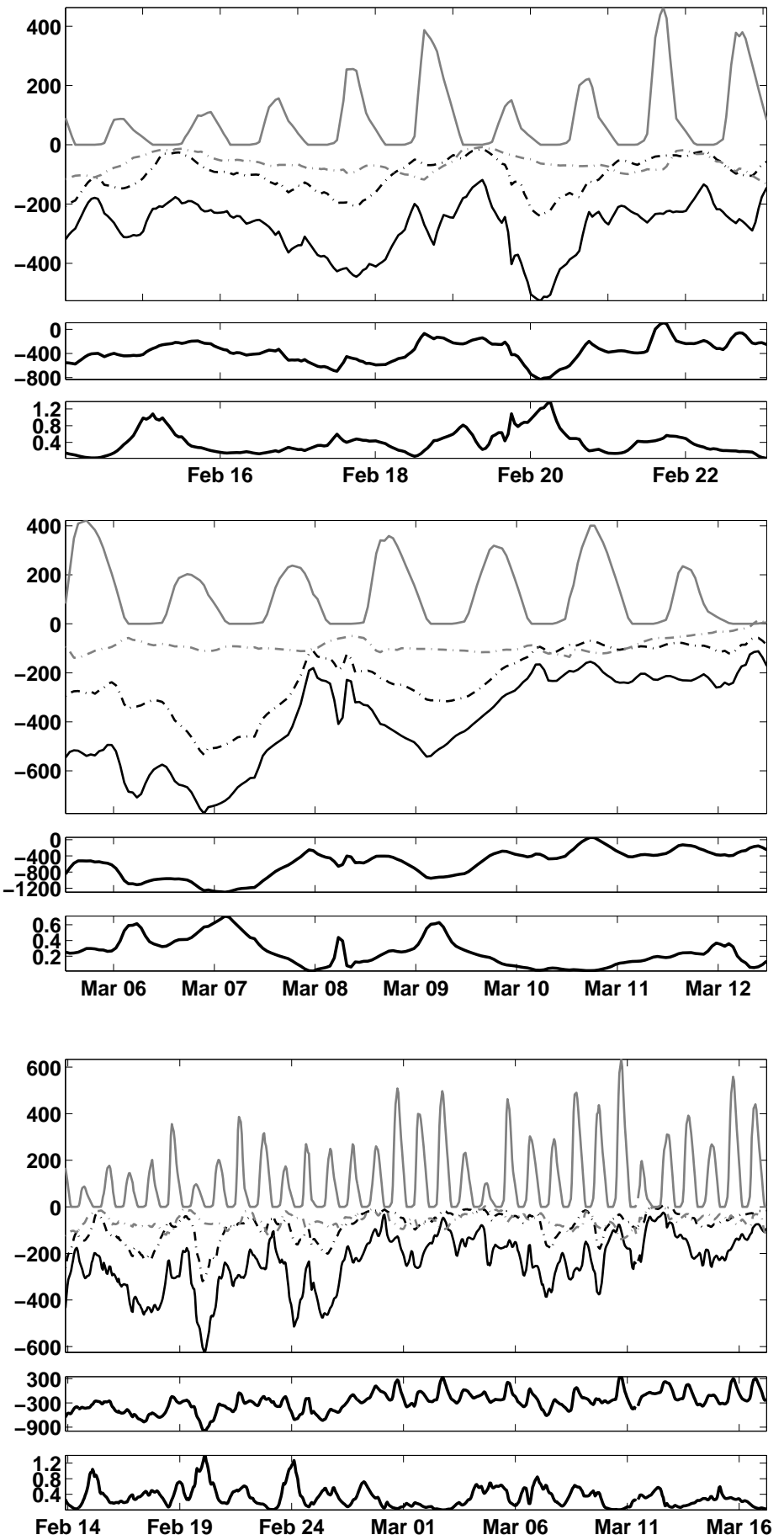

Figure 4-1: Surface forcing following the FILIS (top), EM-APEX 1633 (middle), EMAPEX 1636 (bottom). Within each figure group, the top panel shows the shortwave (gray solid), longwave (gray dashed), latent (black solid), and sensible (black dashed) surface heat fluxes in $W^{-2}$, while the middle panel shows the total surface heat flux in $W^{-2}$ and the bottom panel shows the wind stress in $\mathrm{Nm}^{-2}$. 


\subsubsection{FILIS}

Examination of the FILIS temperature record shows cooling of the upper ocean which was quite intense for the first six days of the deployment with a corresponding deepening of the mixed layer, Figure 4-2a. After this interval, there was a sudden apparent restratification with accompanying rapid shoaling of the mixed layer depth. For the remainder of the FILIS deployment, the upper ocean cooled as the mixed layer again deepened.

Although the temperature record shows cooling for almost the entire FILIS deployment (as does the surface heat flux), examination of the heat storage rate shows that the ocean heat content change during the first six days of deployment is larger than can be accounted for by the estimated net surface heat flux. For the next two days, there is cooling by the surface heat flux, while the heat storage rate indicates that the upper ocean is warming. The only period of time when it seems there might be a balance between net surface heat flux and upper ocean heat storage rate is during the last two days of the FILIS deployment.

\subsubsection{EM-APEX 1633}

The upper ocean exhibited rapid cooling which was accompanied by a deepening mixed layer for the first two days of the EM-APEX float 1633 deployment, Figure 4-2b. The remaining four days of the deployment were not marked by strong changes in either upper ocean temperature or mixed layer depth. The EM-APEX float 1633 mixed layer depth is less variable than those observed by the other two instruments.

Although the surface heat flux and the heat storage rate curves have similar shapes, the heat storage rate exhibits more cooling than can be accounted for by the surface heat flux for nearly all of the EM-APEX 1633 float deployment. Like the FILIS, it is only during the last day or so of the deployment that the surface heat flux and heat storage rate were within reasonable range of each other. 

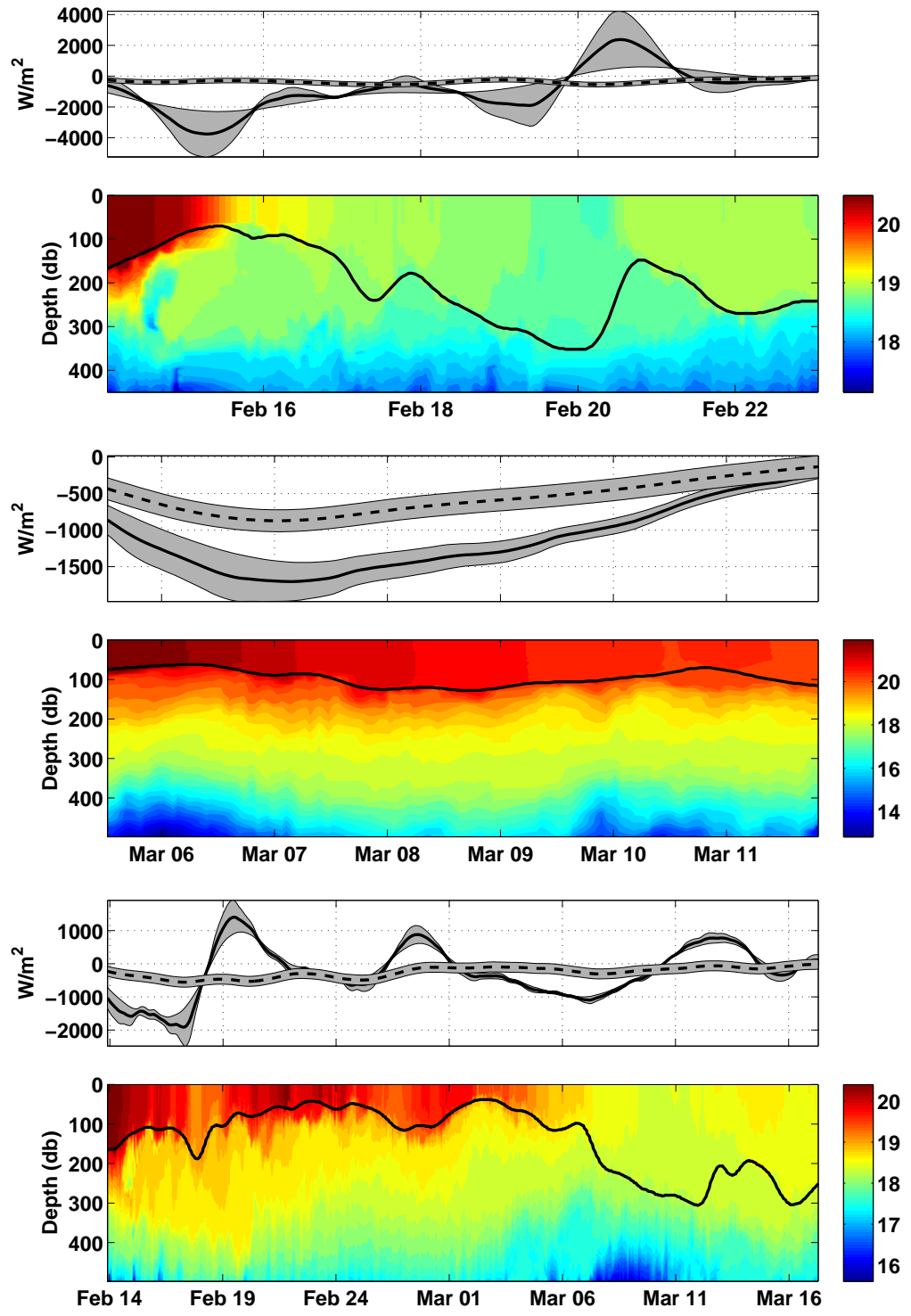

Figure 4-2: One dimensional heat budget results for FILIS (top), EM-APEX 1633 (middle), and EM-APEX 1636 (bottom). For each instrument, the top panel shows surface heat flux (dashed line) and mixed layer heat content (solid line) in $\mathrm{Wm}^{-2}$. The gray area represents the error. The bottom panel displays potential temperature in ${ }^{\circ} \mathrm{C}$ with mixed layer depth (black line) overlaid. Quantities in the top panel were smoothed with a 48 hour running average filter. 


\subsubsection{EM-APEX 1636}

The EM-APEX float 1636 had the longest deployment time of the three instruments, with observations spanning more than a month. Unlike the other two systems, the net surface heat flux was not negative (cooling the ocean) for the entire deployment, although it was for the first twelve days. After that, the surface heat flux oscillated between heating and cooling, with peak amplitudes reaching over $200 \mathrm{Wm}^{-2}$. Like the other systems, the float 1636 upper ocean temperature record shows rapid cooling for the first few days of the deployment, Figure 4-2c. This was followed by almost three weeks of alternating upper ocean warming and cooling. The mixed layer depth remained relatively constant during this time, and then deepened rapidly during two cooling events towards the end of the deployment.

The surface heat flux does not account for the observed mixed layer heat storage rate until possibly the last days of the deployment, which is consistent with the other EM-APEX float and the ASIS-FILIS. Prior to that, the cooling and heating events that dominate changes in heat storage are much too large in magnitude to be accounted for by the surface heat flux.

\subsubsection{FILIS/EM-APEX 1636 comparison}

The ASIS-FILIS and EM-APEX 1636 were deployed in close proximity to one another, and therefore provide a unique perspective for examining the spatial variation of upper ocean response to strong wind and buoyancy forcing events. Examining individual strong forcing events can also provide more insight into the processes controlling the evolution of upper ocean heat storage. In particular, there was a storm passage of about one day duration on February 19-20th, 2007 in which the ASIS-FILIS and EMAPEX 1636 experienced similar buoyancy and wind forcing, Figure 4-3. The wind stress reached over $1 \mathrm{Nm}^{-2}$ during the event, and the surface heat flux was negative (ocean cooling) for the entire period shown, with a peak around $-800 \mathrm{Wm}^{-2}$. The heat storage rate for both the EM-APEX 1636 and the FILIS display values 2-4 


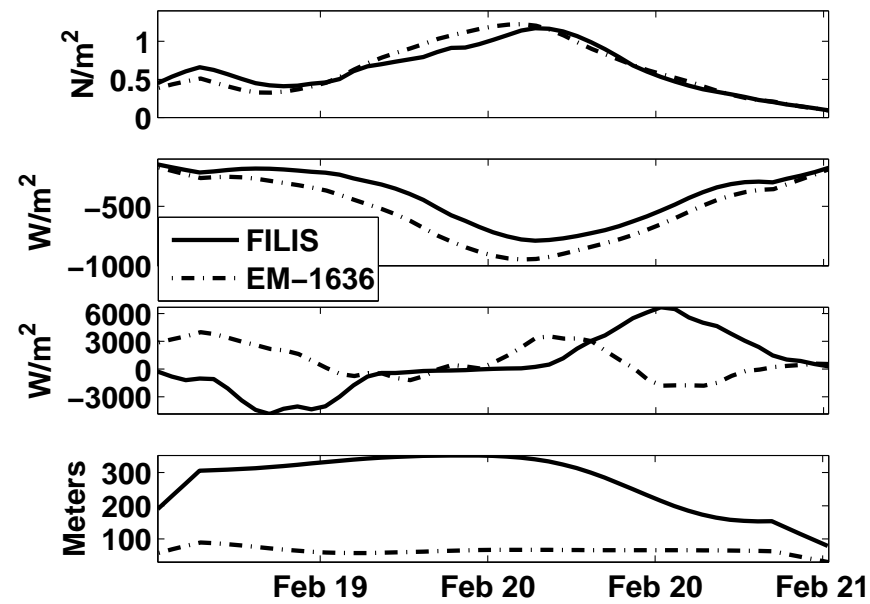

Figure 4-3: Wind stress(top panel), surface heat flux (second panel), heat storage rate (third panel), and mixed layer depth (bottom panel) for a single storm passage in February 2007 for both the EM-APEX 1636 and ASIS-FILIS floats. The data have been smoothed with a running 8 hour mean.

times greater than the surface heat flux with dissimilar time evolution during the storm. The initial mixed layer depth for FILIS was much deeper ( $\sim 300$ meters) than that observed by EM-APEX 1636, and shoaled significantly directly after the storm passage. The EM-APEX 1636 mixed layer didn't change substantially over the time period of the storm passage.

\subsubsection{PWP mixed layer model}

The Price Weller Pinkel (PWP) one-dimensional mixed layer model was used to provide another perspective on the response of the upper ocean to the observed wind and buoyancy forcing (Price et al., 1986). The PWP model consists of a slab-like mixed layer over a stratified interior, with vertical mixing occurring in response to critical static instability, bulk Richardson, and/or gradient Richardson number criteria. Model runs were initialized with the float observed velocity (with time mean removed) and density vertical profiles, and forced with the NAM derived buoyancy and wind forcing at hourly temporal resolution. Changes in the mixed layer heat 
content in the PWP model are by definition due only to the surface forcing and vertical mixing. Therefore, differences between the model output and the observations highlight the effects of relative advective processes. Mixed layer depth is estimated from the model using the same definition that was applied to the data, and the mixed layer temperature is derived as the vertical mean of the potential temperature profile from the surface to the mixed layer depth.

Over the course of the week-long float deployment, the EM-APEX float 1633 observed mixed layer temperature cools by almost $2{ }^{\circ} \mathrm{C}$, with some slight warming events overlaid on this cooling trend, Figure 4-4a. In contrast, the modeled mixed
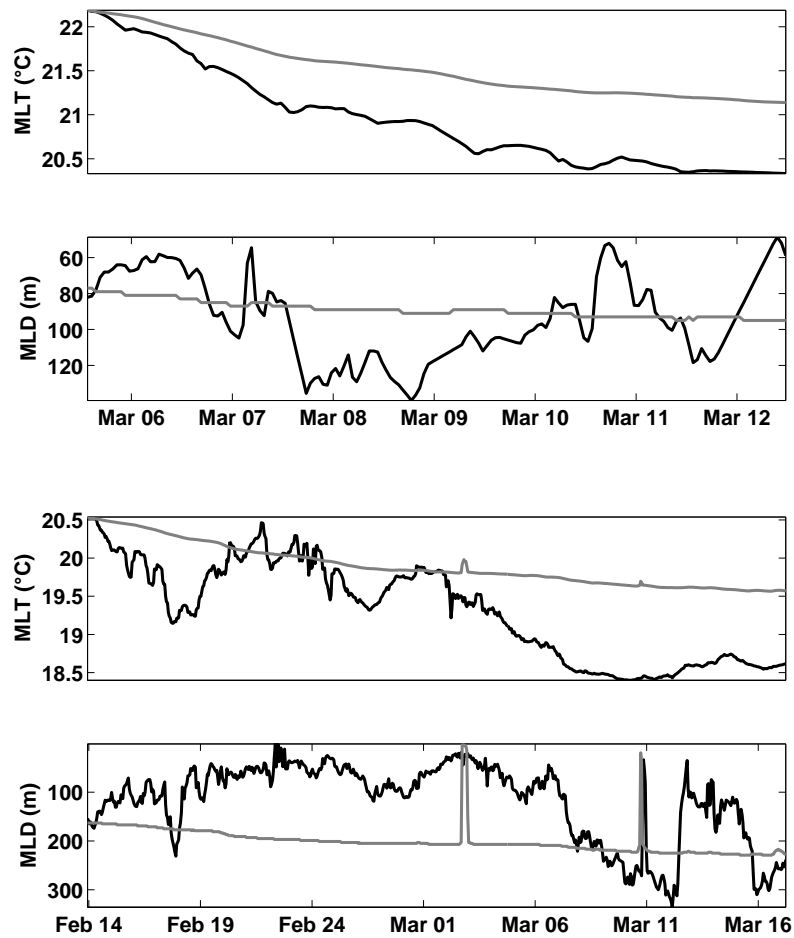

Figure 4-4: PWP model results for EM-APEX 1633 (top two panels) and EM-APEX 1636 (bottom two panels). For each pair of panels, the top panel shows mixed layer temperature from observations (black) and the PWP model (gray) in ${ }^{\circ} C$, and the bottom panel shows mixed layer depth from observations (black) and the PWP model (gray) in $m$.

layer temperature cools by about $1^{\circ} \mathrm{C}$, with only cooling occurring for the duration 
of the float deployment. The observed mixed layer depth is more variable than the modeled one; the maximum observed mixed layer depth was about 140 meters, while the maximum modeled mixed layer depth was 95 meters.

The EM-APEX float 1636 observed mixed layer temperature is also more variable than the modeled mixed layer temperature, Figure 4-4b. The model shows almost uniform cooling for the month-long period, while the observed mixed layer temperature has alternating periods of cooling and warming. Like float 1633, there is more observed cooling by about a degree Celsius over the course of the float deployment than the model predicts. The modeled mixed layer was deeper than that observed for almost the whole deployment. There are two notable restratification events which occur in the model record; the one on March 11th can also be seen in the observed mixed layer depth.

\subsection{Advective effects}

It is clear from the one-dimensional heat budget and modeling results that there is one or more missing terms in the ocean heat budgets following the drifting instruments which are as large as or larger than the surface heat flux. The unaccounted terms remaining in the heat budget equation are the relative advection of the depth-mean temperature by the depth-mean velocity and those involving mixing and entrainment at the base of the mixed layer. This section will deal with the first of these terms, which will be referred to as the depth mean advection for simplicity. It will be argued in the following section that the mixing and entrainment at the base of the mixed layer are negligible in comparison with the depth mean advection.

Although the EM-APEX floats are drifting with the flow, they are also profiling vertically into and out of the mixed layer. This means that there is a small velocity of the float relative to the mixed layer, and therefore the depth mean advection is a potentially significant contributor to the heat budget. This relative flow is a consequence of the vertical current shear where the surface waters are moving at a 
different rate than the water at the base of the float profiles ( $\sim 500$ meters). Examination of the EM-APEX float observed current profiles reveals current differences as large as $50 \mathrm{cms}^{-1}$ over a 50 meter depth range, Figure 4-5, and also shows that the EM-APEX 1636 observed current shear decreased in magnitude over the course of the deployment. Because of the vertical shear present in the horizontal velocities, a
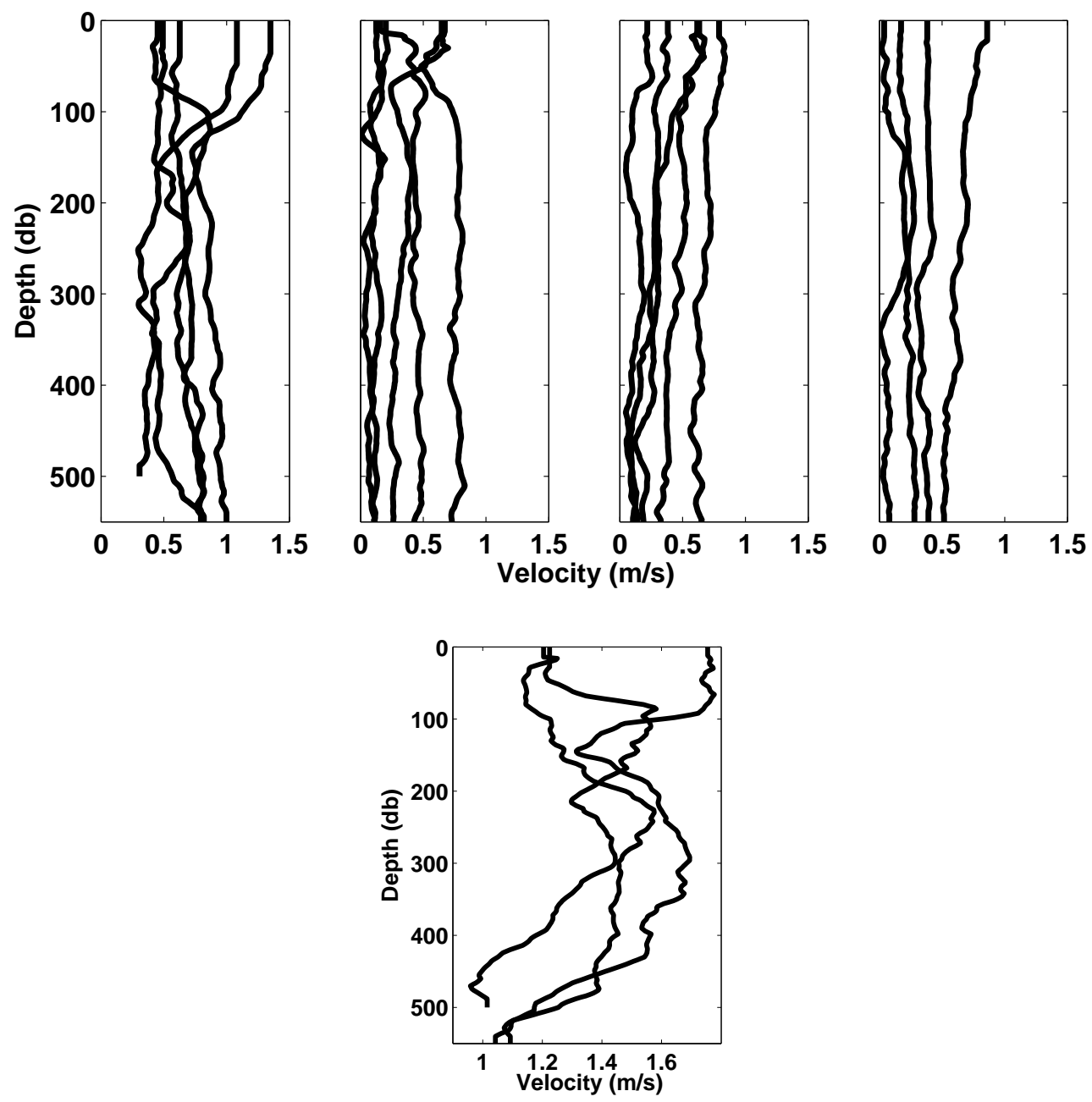

Figure 4-5: Current magnitude profiles for EM-APEX floats 1636 (top) and 1633 (bottom) in $m s^{-1}$. The four top panels each represent one week duration, with the panels being in consecutive time order. Each individual line is separated by one day.

given mixed layer water parcel and a float will travel different distances during the time of a down then up profile set. The distance the float travels is known from 
its position fixes, and the distance the mixed layer water parcel travels is estimated from the absolute velocity profiles as the depth mean velocity of the layer integrated over time. The difference between the float travel distance and the mixed layer water parcel travel distance divided by the time interval gives the relative velocity of the layer to the float. Between up/down profile pairs, when the float is drifting on the surface communicating with the satellite, the relative velocity is estimated as the difference between the distance the float travels on the surface and the mean mixed layer velocity (obtained from the previous profile) integrated in time divided by the time interval.

Like the EM-APEX floats, the ASIS/FILIS one dimensional heat budget is also carried out using profiling observations in the large shear of the Gulf Stream. But, in this case, estimating the relative velocity for the ASIS/FILIS system is different than the EM-APEX floats because the ASIS/FILIS has a large surface component. Both wind and surface waves as well as the tethered FILIS system can drag on the ASIS buoy and prevent it from tracking the surface water perfectly. The FILIS did not return any velocity measurements, but the ASIS buoy had an ADCP mounted on it which provides a direct estimate of the relative velocity. The relative velocities for the ASIS/FILIS and EM-APEX floats are on the order of tens of $\mathrm{cms}^{-1}$, and in the case of EM-APEX float 1636, are larger near the beginning of the deployment than the end, which is consistent with the fact that there was larger shear near the beginning of the deployment, Figure 4-6. There are two components of the depth mean advection: along-stream advection and across-stream advection. Although the EM-APEX alongstream and across-stream relative velocity components (not shown) are of similar magnitude, it is expected that across-stream advection has larger magnitude than along-stream advection because temperature gradients along the Gulf Stream are much smaller than those across the Gulf Stream. The most difficult part about estimating the depth mean advection term in the heat budget is calculating the depth mean lateral temperature gradients about the floats as they drifted. Although there is now precedence for using satellite SST data to estimate heat budget advection 


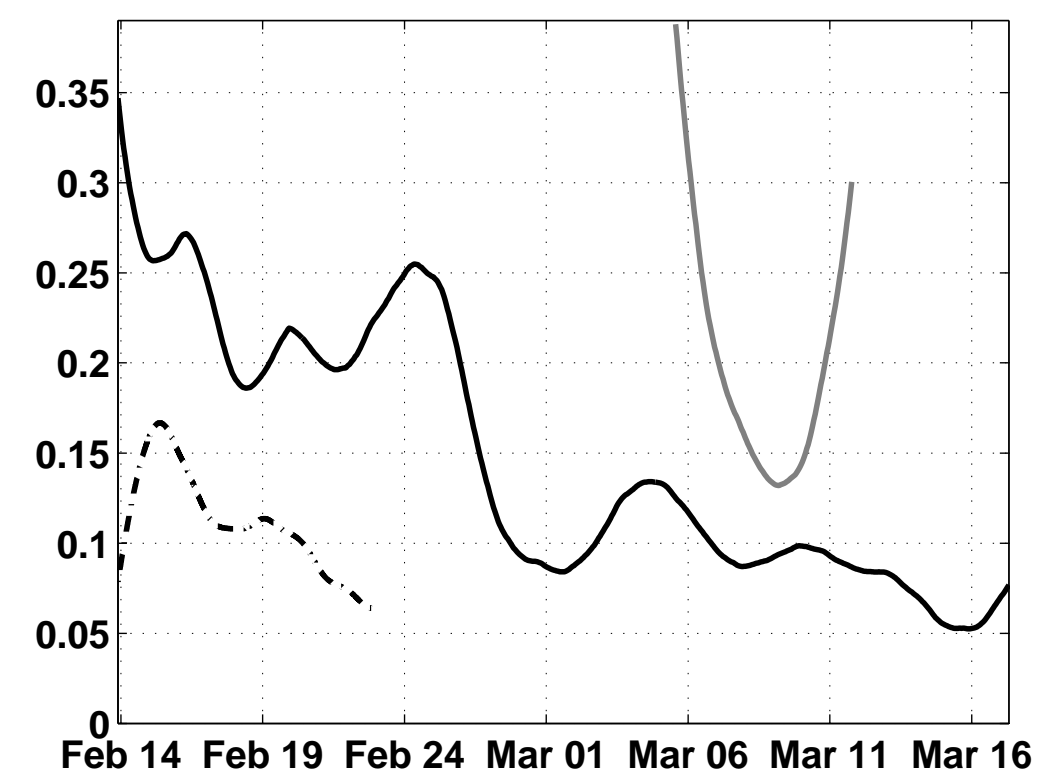

Figure 4-6: EM-APEX 1633 (gray line), EM-APEX 1636 (black line), and ASIS/FILIS (black dotted line) relative velocities in $\mathrm{cms}^{-1}$. All the velocities have been smoothed with a 48 hour running averaging filter.

terms (Foltz and McPhaden, 2005), after exploring this option and comparing to the SeaSoar data, it was determined that the spatial resolution of the satellite SST products is not adequate to capture the mesoscale spatial variation of the temperature field in this region. The majority of the SeaSoar data from the CLIMODE cruises are not close enough to the floats in either space or time to yield a direct estimate of the depth mean advection, but they do provide some information about the size of the temperature gradients in the float drift region. Mesoscale gradients of the depth-mean temperature from the surface to 100 meters were estimated for three of the CLIMODE Seasoar surveys. The mean temperature gradient for this time was $0.06{ }^{\circ} \mathrm{Ckm}^{-1}$, which, when combined with a typical relative velocity of $15 \mathrm{cms}^{-1}$ and the layer depth of 100 meters, gives an advective heat flux divergence of $3500 \mathrm{Wm}^{-2}$. Depth mean advection events of this size would be more than enough to account for the discrepancy between the surface heat flux and the observed heat content.

There were a few SeaSoar sections which spanned float tracks, and returned across- 
stream high horizontal spatial resolution $(\sim 2 \mathrm{~km})$ estimates of salinity, temperature, and pressure. These can be used to directly estimate the across-stream component of the depth mean advection term. In order to get an estimate of the temperature gradient, a spatial scale must be chosen. For this study, the spatial scale of the temperature gradient is estimated as product of the time period which we are smoothing the heat budget terms (48 hours) and the relative velocity. Using the mean relative velocity gives a length scale on the order of ten kilometers. The direct estimation of the across-stream depth mean advection term was limited to a few points where the SeaSoar data were near the track of EM-APEX float 1633 within a day of the float's passage. Those depth mean advection estimates (which have a mean uncertainty of $\pm 120 \mathrm{Wm}^{-2}$ ) show good agreement with the residual needed to close the one dimensional heat budget, Figure 4-7.

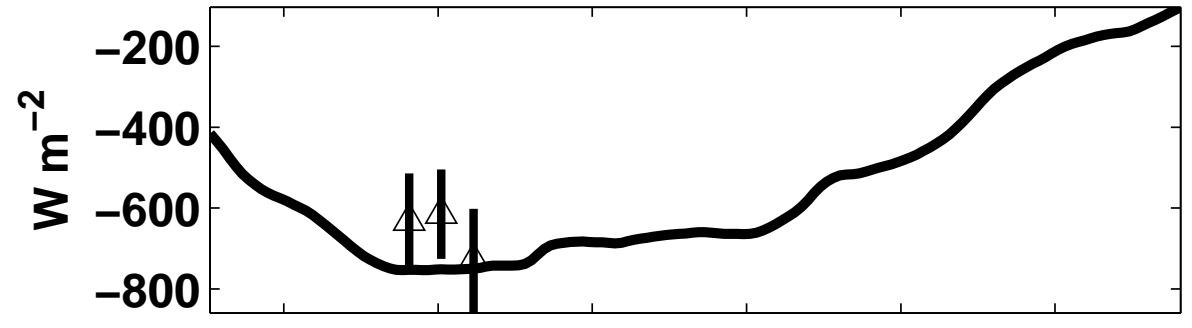

$\begin{array}{llllllll}03 / 06 & 03 / 07 & 03 / 08 & 03 / 09 & 03 / 10 & 03 / 11\end{array}$

Figure 4-7: EM-APEX 1633 1D heat budget residual (solid line) and estimates of the depth mean advection contribution(triangles)

\subsection{Mixing and entrainment at the base of the mixed layer}

The other processes which can potentially contribute to the mixed layer heat budget are mixing and entrainment. Vertical heat fluxes across the mixed layer base associ- 
ated with mixing can alter the mixed layer heat storage. In addition, as the mixed layer deepens, water in the stratified interior is entrained into the mixed layer, also altering the mixed layer heat storage. The EM-APEX and FILIS observations do not provide a way to directly estimate this term. However, the Richardson number can be estimated, and used as a proxy to determine regions where mixing might potentially be taking place. Because the FILIS did not return velocity data, Richardson number profiles were derived only from the EM-APEX float deployments. The observed potential density and velocity profiles were first smoothed over a 10 meter depth interval, and then used to calculate the Ri number with the following expression:

$$
R i=\frac{\frac{-g}{\rho_{o}} \frac{\Delta \sigma_{\theta}}{\Delta z}}{\left(\frac{\Delta u}{\Delta z}\right)^{2}+\left(\frac{\Delta v}{\Delta z}\right)^{2}}
$$

where $g$ is the gravitational constant $9.8 \mathrm{~ms}^{-2}$, the reference density $\rho_{o}$ is taken to be $1024 \mathrm{kgm}^{-3}$, and $\sigma_{\theta}, u$, and $v$ are the potential density, zonal velocity, and meridional velocity respectively. The mixed layer in both float records tends to have supercritical $(R i \leq 0.25)$ Richardson number because the stratification is very weak, Figure 4-8. It can be seen, especially towards the second half of both float deployments, that the Richardson number was generally not supercritical at the mixed layer base. In fact, float 1633 had a supercritical Richardson number at the mixed layer base for only $14 \%$ of the time, while float 1636 sampled supercritical Richardson number at the mixed layer base $19 \%$ of the time. Microstructure observations taken during CLIMODE cruises suggest that when there was mixing at the base of the mixed layer, the turbulent vertical heat flux was generally on the order of $10 \mathrm{Wm}^{-2}$, although one mixing event was observed with turbulent vertical heat flux as large as $1000 \mathrm{Wm}^{-2}$ associated with a temperature intrusion (Inoue and Gregg, 2008). Therefore, we conclude here that at the infrequent times when mixing was occurring at the base of the mixed layer, the resulting vertical heat flux wasn't large enough to provide the large residuals needed to close the one-dimensional heat budget.

This conclusion is supported by the inability of the one-dimensional PWP mixed 

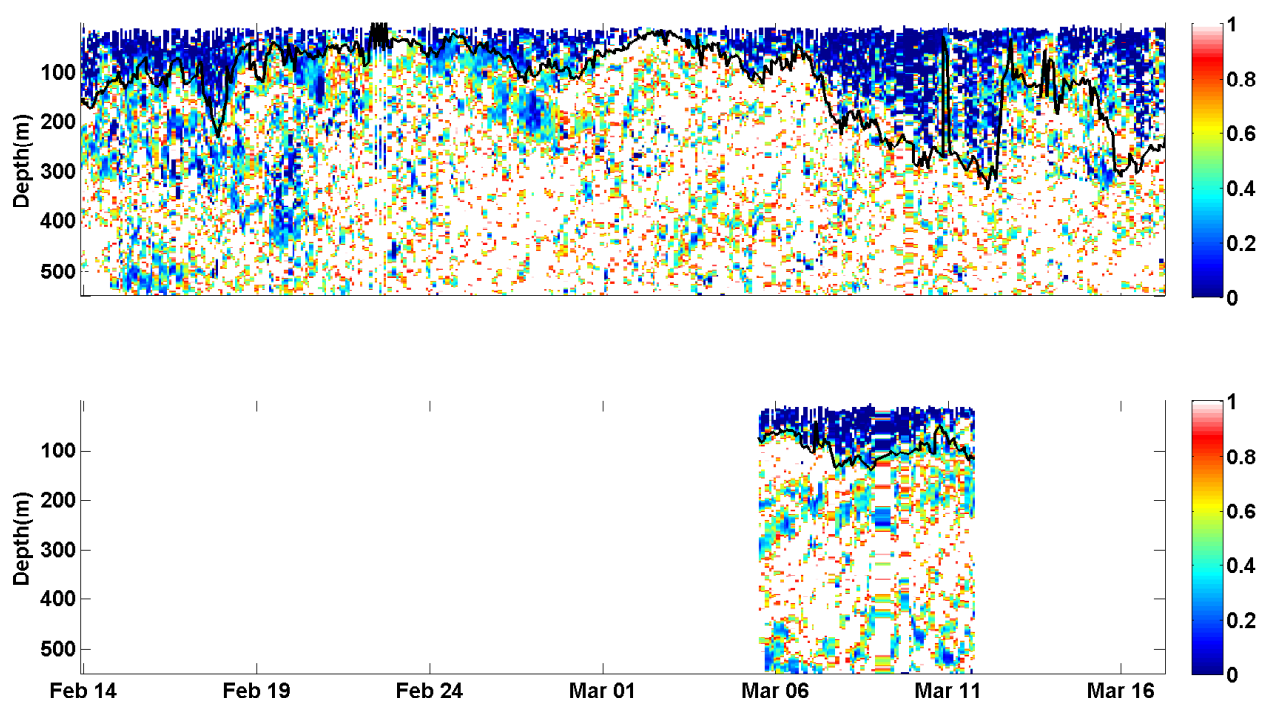

Figure 4-8: Richardson number for EM-APEX 1636 (top) and EM-APEX 1633 (bottom) with mixed layer depth overlaid (black line).

layer model, which has a representation of vertical mixing processes, to capture the observed evolution of the mixed layer for either float. The PWP model results can also be used to obtain an estimate of the vertical heat flux due to the mixing at the base of the mixed layer. The model heat storage rate was estimated using the same method that was used for the observations. The vertical mixing is derived as difference of the heat storage rate and the surface heat flux. The resulting mixing term is then smoothed using the 48 hour running averaging filter to mimic the observational heat budget results. The vertical mixing term was found to be much smaller in magnitude than the CLIMODE observed heat fluxes and the observational one-dimensional heat budget residuals; the time mean vertical mixing term was $-11 \mathrm{Wm}^{-2}$ and $-16 \mathrm{Wm}^{-2}$ for the 1636 and 1633 floats respectively. 


\subsection{Discussion}

Examination of observations (taken with drifting profiling instruments deployed in the Gulf Stream) of upper ocean heat storage rate during strong surface heat flux $(>1000$ $\left.W \mathrm{~m}^{-2}\right)$ and wind $\left(>1 \mathrm{Nm}^{-2}\right)$ forcing yielded unexpected results. Quasi-Lagrangian one-dimensional heat budgets exhibited large discrepancies between the observed changes in mixed layer heat storage and the net surface heat flux. The observed residual was at times as large as twice the observed surface heat flux. This residual can be accounted for by a displacement advection due to the small relative velocity of the floats and mixed layer water parcels as the floats cycle vertically in the large current shear and the large temperature gradients found in the Gulf Stream region.

In theory, quasi-Lagrangian heat budgets using drifting instrumentation, like the study presented here, reduce the advection terms; in practice, however, these kinds of experiments have had mixed success with limiting advective effects. A heat budget study using drifting thermistor chains in the North Pacific Ocean during fall showed that, when averaged over several storms, the advection term was negligible, but that it could be important on shorter time scales (Large et al., 1986). Examination of drifting buoy data from the western equatorial Pacific in preparation for TOGA-COARE yielded budget closure within $25 \mathrm{Wm}^{-2}$ without accounting for advection, and it was concluded that the technique of using a drifting measurement to reduce advection was successful (Bradley et al., 1993). However, Paduan et al. (1988) used a drifting instrument chain off the coast of California, and found advection to be on the same order as the surface heat flux, as did Richards et al. (1995), who directly estimated advection by performing ship surveys around a drifting buoy in the equatorial Pacific Ocean.

Because the advection term estimated for this quasi-Lagrangian study is a relative advection term, we can assume that performing an Eulerian heat budget under the same circumstances would yield advection of an even greater magnitude that is capable of dominating over the strong surface forcing. This assertion is corroborated by 
previous model fixed volume heat budgets of the Gulf Stream region that suggested advection could be as large as the surface heat flux (Kelly and Qui, 1995; Dong and Kelly, 2004). However, Eulerian observations of upper ocean heat content changes during a wintertime storm passage over the Gulf Stream off the coast of South Carolina demonstrate that the heat budget is dominated by the strong buoyancy forcing associated with the storm (Xue et al., 1994). Xue et al. (1994) suggest that the discrepancies between their results and the results of Kelly and Qui (1995); Dong and Kelly (2004) are related to the fact that after the Gulf Stream has separated from the coast, and is flowing east-west (as in Kelly and Qui (1995); Dong and Kelly (2004)), along stream westerly winds can shift the Stream southwards, leading to a large Ekman advection contribution to the heat budget. Additionally, there a few possible explanations for the discrepancies between this study and the results of Xue et al. (1994). First, Xue et al. (1994) neglect both shortwave and longwave surface heat fluxes in their calculation, which could lead to an overestimation of the net surface heat flux, and therefore an underestimation of the one-dimensional heat budget residual. Secondly, the calculation Xue et al. (1994) use is a volume budget which is integrated in depth and the across-stream direction, in contrast to the calculation presented here which is only integrated in depth. For a random velocity field, there should be advection which heats and cools the upper ocean in different places, and so by integrating in the across-stream direction, Xue et al. (1994) are estimating an advection term which is potentially reduced in comparison to the advection term estimated for this study.

The results of this experiment suggest that future drifting studies of the heat budget in the Gulf Stream region will have to take advection into account, even under strong wintertime buoyancy forcing conditions. Given the large temperature gradients in the region, modest flows of $10-20 \mathrm{cms}^{-1}$ can result in advective heat fluxes which are three times as large as the surface heat flux. High horizontal resolution temperature measurements are necessary in order to get an accurate estimate of the temperature gradients to calculate advection. Longer term measurements would also be useful in 
order to allow the possibility of temporal averaging, which may reduce influence of the advection term. For profiling floats, reducing the depth range of the profiling will lessen the relative velocity, which in turn will reduce the magnitude of the relative advection. Using the EM-APEX velocity records, the relative velocity was estimated for varying profiling depths. That is, for a chosen profiling depth, the velocity profiles were integrated down to that depth. The distance the float traveled in the time the float takes to profile to the chosen depth was subtracted from the distance a water parcel moving at the mean mixed layer velocity would travel. This difference in distances is then divided by time to give the relative velocity. The advective heat flux was then determined using a typical mixed layer depth (100 meters) and temperature gradient $\left(2.7 \times 10^{-5}{ }^{\circ} \mathrm{Cm}^{-1}\right)$, and a time mean was taken. The profiling depth which minimizes the displacement advective heat flux is approximately 70 meters, Figure 4-9. In order for the advective heat flux to remain below the error in the surface heat flux $\left(150 \mathrm{Wm}^{-2}\right)$, the float must not profile deeper than 125 meters. 


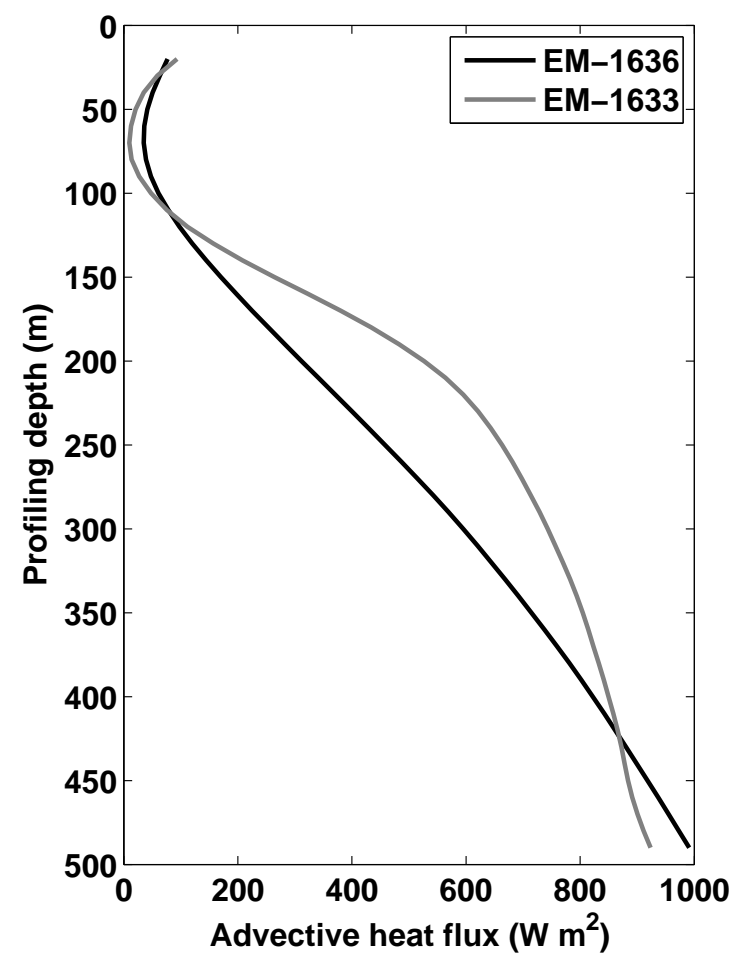

Figure 4-9: Advective heat flux for varying profiling depths in $W^{-2}$ for EM-APEX 1633 (gray) and EM-APEX 1636 (solid). 


\section{Chapter 5}

\section{The influence of near-inertial wave trapping in the Gulf Stream on the upper ocean stratification}

\subsection{Introduction}

The passage of wintertime storms carrying cold air over the warm Gulf Stream leads to energetic surface heat transfer from the ocean to the atmosphere accompanied by strong upper ocean convection. It has long been hypothesized that this results in the formation of a weakly stratified water mass known as Eighteen Degree Water (EDW) (Worthington, 1959). Discrepancies between EDW formation rates estimated directly from upper ocean observations and those calculated from air-sea buoyancy fluxes motivated the CLIvar MOde water Dynamics Experiment (CLIMODE), which aims to resolve the issue through multiple observations of the Gulf Stream during winter. Wintertime storms bring not only strong buoyancy forcing, but strong wind forcing as well, which can potentially complicate the EDW formation process. Thomas and Marshall (2005) suggest that down-front winds along the Gulf Stream can result in an ageostrophic circulation which advects cold water over warm, and results in EDW formation due to convective mixing. Here, we discuss another possible mechanism 
through which wind energy can be transmitted through the ocean and result in weakly stratified water in the EDW formation region; wind-forced near-inertial motions can become trapped in negative vorticity regions, ultimately breaking and dissipating locally.

As has been often observed, wind forcing which is variable in time can force mixed layer oscillations at the inertial frequency. In general, a portion of the energy imparted from the wind into near-inertial motions eventually propagates down into the stratified interior in the form of near-inertial internal waves. These near-inertial internal waves appear to be able to propagate equatorward for long distances, spreading the wind-forced energy out across the ocean before it is ultimately dissipated (Garrett, 2001). However, modeling and observational studies have demonstrated that nearinertial motions (both mixed layer oscillations and internal waves in the stratified interior) can be affected by a strong relative vorticity signal in the mesoscale background field, such as the Gulf Stream. This interaction can result in an altered propagation path for the near-inertial energy.

Observations have shown that mixed layer near-inertial motions can decay very rapidly when the relative vorticity gradient of the background flow field is large, regardless of whether the vorticity is positive or negative, (Meurs, 1998). This conclusion has been supported by a number of modeling studies (Balmforth and Young, 1999; Young and Jelloul, 1997). The decrease in mixed layer decay time comes about because the mesoscale ocean structure imparts a smaller horizontal spatial scale on the mixed layer oscillations than the wind forcing. In the open ocean in the absence of a background flow, the horizontal spatial scale of near-inertial motions is set by characteristics of the wind forcing (such as storm size and speed) and is generally on the order of hundreds of kilometers; however in the presence of mesoscale structures (such as the Gulf Stream front) this scale is typically reduced to tens of kilometers. This leads to rapid transfer of near-inertial energy to higher vertical modes, and therefore rapid vertical propagation of the near-inertial energy because the vertical group speed of internal waves is inversely dependent on horizontal wavenumber. This 
implies that regions with significant vorticity gradients are areas where near-inertial energy can radiate out of the mixed layer very efficiently.

Below the mixed layer, the effective wave frequency (defined in equation 3.1 in Chapter 3 of this thesis and rewritten here for convenience) of near-inertial internal waves propagating in negative vorticity regions can be less than the local resting inertial frequency:

$$
f_{\text {eff }}^{2}=f_{0}\left(f_{0}+\zeta\right)=f_{0}\left(f_{0}+\left[\frac{\partial V}{\partial x}-\frac{\partial U}{\partial y}\right]\right)
$$

As frequency depressed near-inertial waves radiate downwards, they are reflected by horizontal turning points at the locations where their intrinsic frequency equals the local effective frequency (Kunze, 1985). As these waves approach a region in the vertical where the intrinsic frequency equals the local effective frequency, known as a critical layer, the amplitude of the trapped waves grows and the vertical wavelength shrinks, resulting in locally enhanced near-inertial shear, and therefore overturns which can homogenize localized regions of the density structure. Modeling studies have confirmed the theoretical prediction that near-inertial energy can be enhanced in regions of negative relative vorticity and penetrate deeper than in regions of negligible relative vorticity (Danioux et al., 2008). Because the EDW formation is thought to occur on the southern edge of the Gulf Stream, which is the negative vorticity side of the jet, this near-inertial wave trapping mechanism can result in weakened stratification which could potentially precondition the water column for or interfere with mode water formation. The dissipation of trapped near-inertial waves in regions of negative vorticity has been highlighted in both observational and modeling studies. Kunze et al. (1995) found enhanced levels of turbulent dissipation near a vertical critical layer in a warm core Gulf Stream ring. And, numerical simulations of both cyclonic and anti-cyclonic eddies determined that there were times when the vertical dissipation dominated the kinetic energy budget in a critical layer in a negative vorticity core (Lee and Niiler, 1998). 
Observations presented in Chapter 3 of this thesis described the influence of mesoscale vorticity on near-inertial motions during the CLIMODE program. The EM-APEX observed velocity record had a large near-inertial component, and use of the extended complex demodulation technique revealed that the near-inertial frequency appeared to be shifted by the presence of mesoscale relative vorticity (as observed using the shipboard ADCP), suggesting that the relative vorticity was large enough to trap near-inertial waves. The limitation of the available CLIMODE data is that further investigation of the propagation of near-inertial waves in the Gulf Stream frontal structure cannot be done, as the floats drifted out of the Gulf Stream jet after a short time. Consequently, a numerical analysis study was carried out to investigate the trapping of near-inertial waves in regions of negative vorticity. The goals of this modeling study were to capture the near-inertial response to wind forcing in a Gulf Stream-like jet, characterize and quantify the resulting enhanced mixing in the negative vorticity region, and assess the impact of the enhanced mixing on the stratification in the EDW formation region.

\subsection{Model implementation}

\subsubsection{DPWP model}

The three dimensional Price Weller Pinkel (3DPWP) model was used to examine the propagation of near-inertial motions in a jet background flow. The 3DPWP model is a three dimensional, hydrostatic, primitive equation model which uses the Price Weller Pinkel (PWP) mixing scheme in the vertical (Price et al., 1986). There are three mixing mechanisms in the PWP model; one based on the gradient Richardson number, one based on the bulk Richardson number which acts solely at the base of the mixed layer, and one requiring the density profile to be statically stable. The PWP vertical mixing scheme has been shown to accurately capture the energy flux from the wind into mixed layer near-inertial motions in one dimensional simulations, because it allows for deepening of the mixed layer in response to wind forcing (Plueddemann and 
Farrar, 2006). The 3DPWP model has been used successfully to model the inertial response of the ocean to hurricane wind forcing (Price, 1983).

\subsubsection{Model setup}

The model was run in a channel configuration on an $f$-plane, initialized with a horizontal density front and corresponding sheared geostrophic velocity. The initial conditions are idealized, but were based on a series of CTD sections taken in the Gulf Stream during the CLIMODE program, Figure 5-1. The maximum jet velocity was
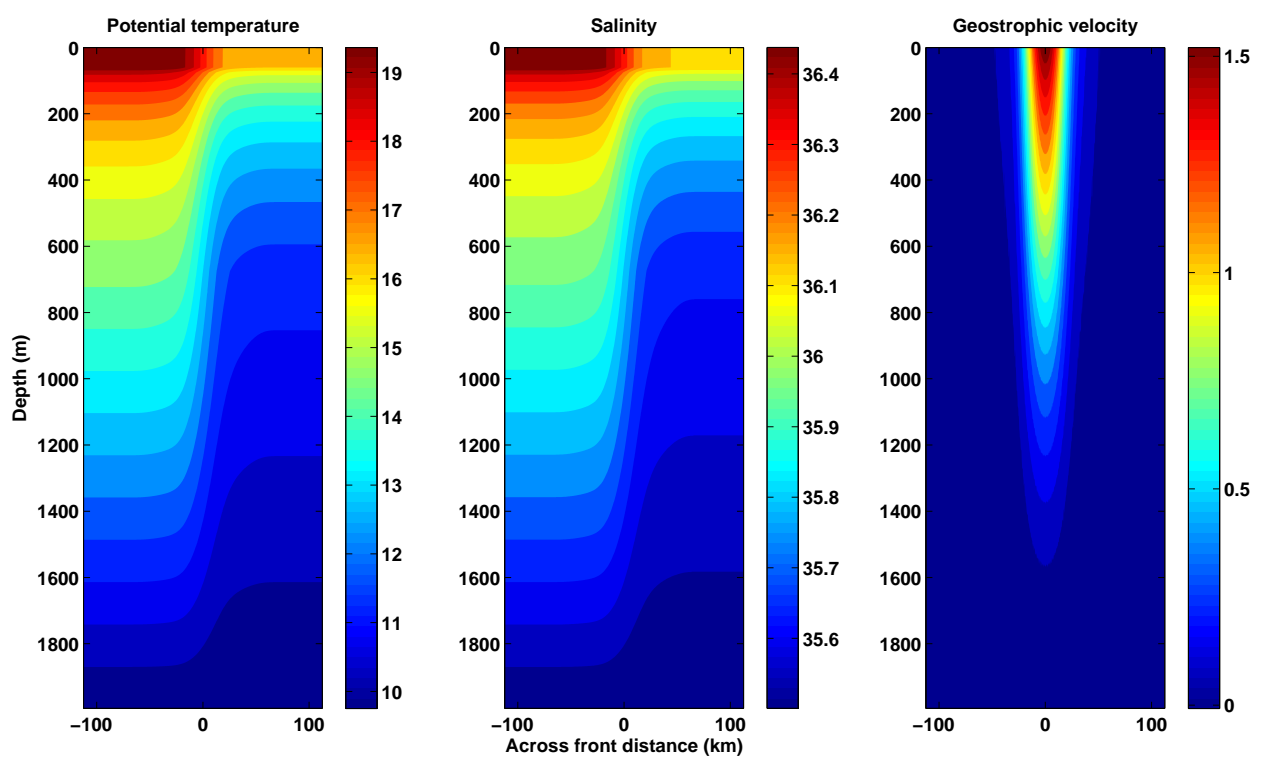

Figure 5-1: Initial potential temperature $\left({ }^{\circ} \mathrm{C}\right)$, salinity $(\mathrm{psu})$, and geostrophic velocity $\left(m s^{-1}\right)$ for the model runs.

approximately $1.6 \mathrm{~ms}^{-1}$, and the jet and front were confined to a central region spanning about 50 kilometers. The vertical density structure was designed to mimic the CLIMODE observations with an initially 60 meters deep mixed layer, a thermocline below, and weakening vertical gradients as the depth increases. The initial effective frequency in the negative(positive) vorticity core was .5 $f(1.5 f)$. The model domain 
is 2000 meters in depth and 225 kilometers in the across front direction, with 15 meter vertical resolution and 250 meter horizontal resolution. The model was run for 20 days with a time step of 50 seconds. The high horizontal and temporal resolution, which are not necessarily required to study near-inertial waves with comparatively large (tens of kilometers) horizontal wavelength and long periods ( 19 hours at the model latitude of $37^{\circ} \mathrm{N}$ ), were deemed necessary to maintain numerical stability of the background density front and corresponding jet. (Initial model runs with coarser spatial and temporal resolution resulted in rapid slumping of the density front and weakening of the geostrophic jet due to numerical diffusion, Figure 5-2.) The model
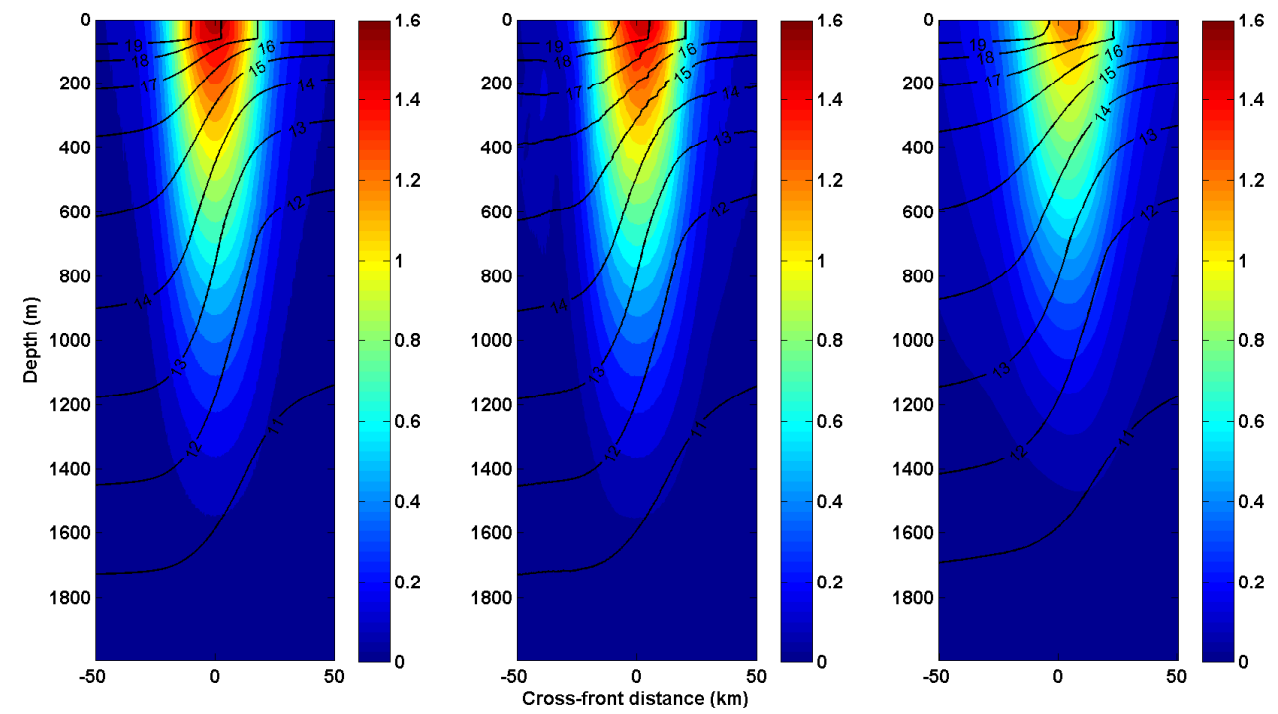

Figure 5-2: Along front velocity (color contours) and temperature (black contours) at the initial step (left panel), the end of a high spatial resolution run (middle panel) and the end of a low spatial resolution run (right panel). Velocities are in $m s^{-1}$ and temperature is in ${ }^{\circ} \mathrm{C}$.

was run with periodic boundary conditions in the along-front direction, making the channel effectively infinite. Because there was no imposed variation in the along-front direction, and none developed during these short integrations, this simulation can be thought of as two-dimensional. Radiation boundary conditions (which parameterize 
the radiation of waves out of the domain at a fixed wave speed) were implemented in combination with a sponge layer at the north and south edges of the model domain. There was also a sponge layer at the bottom to prevent vertically propagating energy from reflecting and reentering the domain.

\subsubsection{Model forcing}

Wind forcing that is rotating at the resting inertial frequency, has an amplitude of $.6 \mathrm{Nm}^{-2}$, and lasts exactly one inertial period (19 hours) is applied uniformly to the whole domain. This is a commonly used forcing in near-inertial studies, because it results in the maximum possible amount of energy input at the inertial frequency and also mimics the passage of an atmospheric cold front system across the model domain (D'Asaro, 1985). However, this forcing does not account for potential feedbacks between the ocean and the atmosphere; it is likely that the presence of the jet/frontal structure in the upper ocean would result in variations in the surface forcing across the front. For the purposes of this study, the forcing was chosen in order to isolate solely the effects of the presence of structure in the ocean. The uniformity of the wind forcing implies that any spatial variability in the model results are due to the horizontal structure of the background ocean flow. Further work done on this subject will need to address the issues of spatially varying surface forcing. The frequency response of the upper ocean in the vicinity of the jet to the rotating wind forcing is discussed in the following section.

\subsection{Influence of relative vorticity on near-inertial motions}

\subsubsection{Frequency response}

The model meridional velocity time series shows strong oscillations in the near-inertial band which occur as a response to the wind forcing, Figure 5-3. It can be seen that 

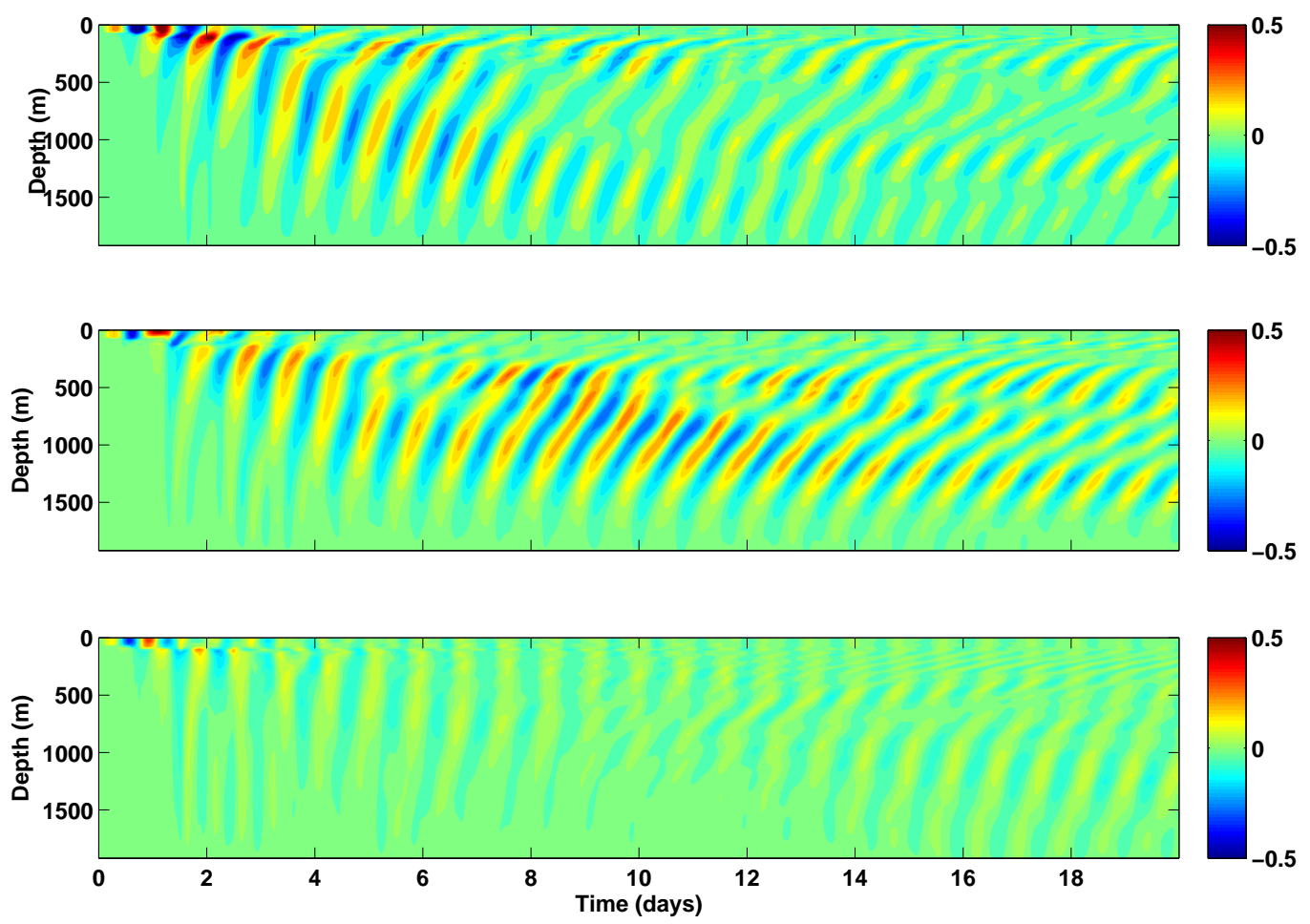

Figure 5-3: Depth/time slices of meridional velocity $\left(\mathrm{ms}^{-1}\right)$ taken from the negative vorticity core (top panel), the center of the jet (middle panel), and the positive vorticity core (bottom panel).

this response varies across the jet, both in amplitude and in frequency. Energetic motions with amplitude around $30 \mathrm{cms}^{-1}$ can be seen in the negative vorticity region. Similarly, the positive vorticity region also exhibits strong oscillations near the surface during the first few days of the model run. However, the interior oscillations are much weaker in the positive vorticity than in the negative vorticity, and the period of the oscillations is clearly longer in the negative vorticity region than in the positive vorticity region, especially at the surface during the forcing. The motions in the center of the jet are the most energetic of the three locations shown.

Surface rotary frequency spectra, which decompose a vector time series into clockwise and counterclockwise rotating energy (Gonella, 1972), were estimated in the center of the jet and confirmed that the most energetic motions are in the near-inertial 
band and rotating clockwise in time, Figure 5-4 (top panel). There is some energy

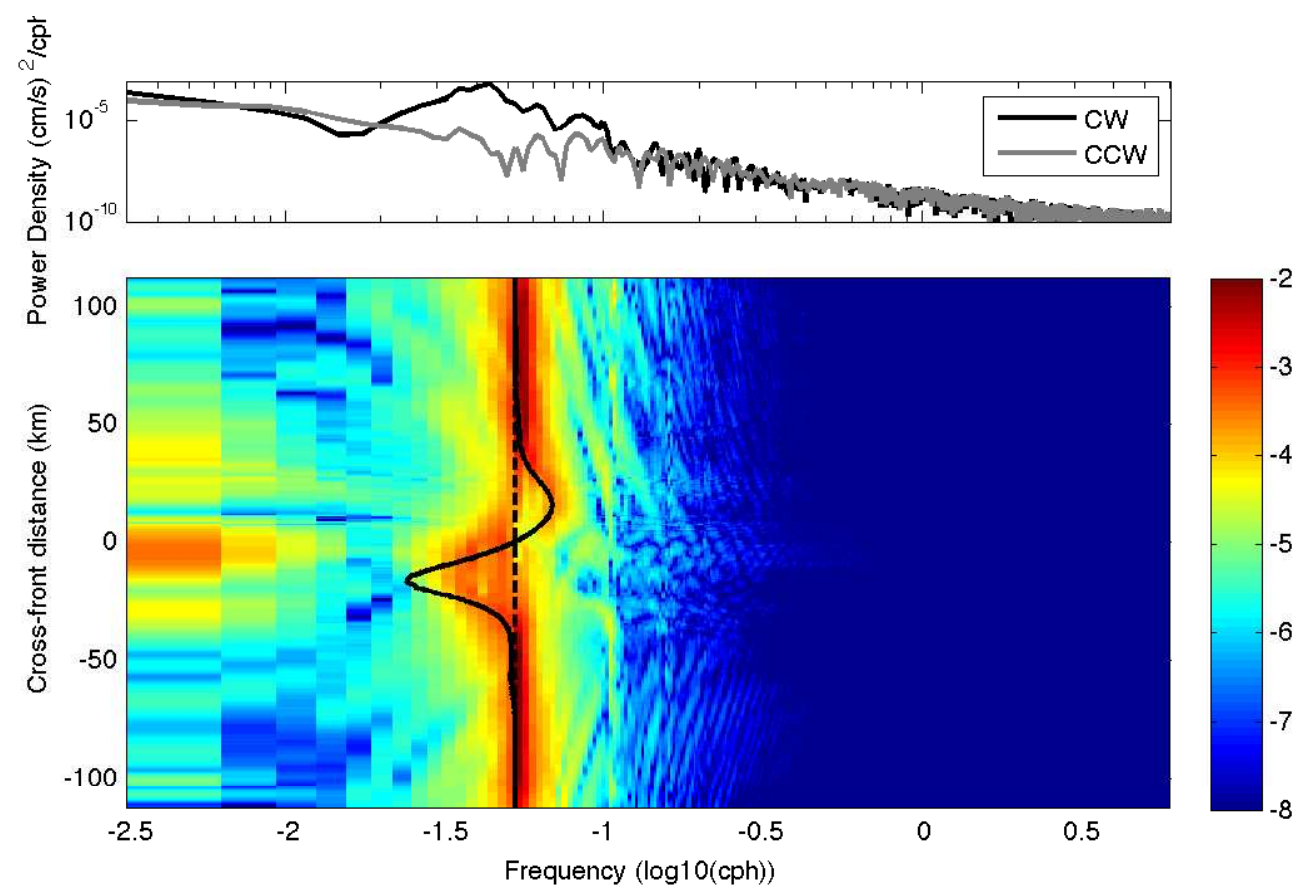

Figure 5-4: Top panel: Rotary frequency spectra components from the surface of the domain at the center of the jet. Bottom panel: Cross-front/frequency section of the clockwise component of the surface rotary frequency spectrum with effective inertial frequency (solid line) and resting inertial frequency (dashed line) overlaid.

contained in sub-inertial frequency motions which could represent evolution of the background flow, but these are less energetic than the near-inertial motions. The cross-front distribution of the clockwise component of the surface rotary frequency spectra reveals shifting of the energy in the inertial band to frequencies less than resting $f$ in the negative vorticity region and frequencies greater than resting $f$ in the positive vorticity region, Figure 5-4 (bottom panel). There is also an energy peak across the whole front at the resting inertial frequency. The oscillations in the negative vorticity region which have frequency below the resting inertial frequency are unable to freely propagate away from the region and are expected to become trapped 
and dissipate in the negative vorticity region. This is the mechanism described by Kunze and Sanford (1984), and illustrated with the ray tracing technique in Figure 16 of that paper.

\subsubsection{Analytical response to forcing}

A better understanding of the near-inertial response of the surface layer to the inertially rotating wind forcing can be obtained by solving a linearized form of the momentum equations analytically. We begin by decomposing the along stream velocity into an Ekman, or wind-forced, component and a geostrophic component, i.e. $u=u_{e}+u_{g}, v=v_{e}$. Assuming that the acceleration, Coriolis, and wind forcing terms dominate during the wind forcing, we can write the momentum equations for the wind-forced motions as

$$
\begin{gathered}
\frac{\partial u_{e}}{\partial t}-(\zeta+f) v_{e}=\frac{-A \cos (f t)}{\rho_{0} h} \\
\frac{\partial v_{e}}{\partial t}+f u_{e}=\frac{A \sin (f t)}{\rho_{0} h}
\end{gathered}
$$

where $A$ is the amplitude of the forcing (here $.6 \mathrm{Nm}^{-2}$ ), $\mathrm{h}$ is the initial mixed layer depth (here 60 meters), and $\zeta=\frac{\partial u_{g}}{\partial y}$ is the relative vorticity. Taking the Laplace transform of the momentum equations yields:

$$
\begin{gathered}
s \hat{u}_{e}-(f+\zeta) \hat{v}_{e}=\frac{A s}{\rho_{0} h\left(s^{2}+f^{2}\right)} \\
s \hat{v}_{e}+f \hat{u}_{e}=\frac{A f}{\rho_{0} h\left(s^{2}+f^{2}\right)}
\end{gathered}
$$

Defining $f_{\text {eff }}^{2}=f(f+\zeta)$, this system of two equations and two unknowns can be solved algebraically for the velocity components, giving

$$
\hat{u}_{e}=\frac{A}{\rho_{0} h} \frac{f_{\text {eff }}^{2}-s^{2}}{\left(s^{2}+f^{2}\right)\left(s^{2}+f_{\text {eff }}^{2}\right)}
$$




$$
\hat{v}_{e}=\frac{2 A}{\rho_{0} h} \frac{s f}{\left(s^{2}+f^{2}\right)\left(s^{2}+f_{\text {eff }}^{2}\right)}
$$

Finally, an inverse Laplace transform is applied to these expressions to give the time evolution of the velocity components.

$$
\begin{gathered}
u_{e}=\frac{A}{\rho_{0} h}\left(\frac{2 f_{e f f} \sin \left(f_{e f f} t\right)}{\left(f-f_{e f f}\right)\left(f+f_{e f f}\right)}-\frac{\left(f_{\text {eff }}^{2}+f^{2}\right) \sin (f t)}{f\left(f-f_{e f f}\right)\left(f+f_{\text {eff }}\right)}\right) \\
v_{e}=\frac{2 A}{\rho_{0} h} \frac{f\left(\cos \left(f_{\text {eff }} t\right)-\cos (f t)\right)}{\left(f-f_{\text {eff }}\right)\left(f+f_{\text {eff }}\right)}
\end{gathered}
$$

Comparison of this analytical solution and the model response during the forcing show good agreement, Figure 5-5. Both velocity component solutions contain sinusoidal terms with arguments at both the inertial frequency for a resting ocean and the effective frequency, which explains why there are peaks at both of those frequencies in the previously shown rotary frequency spectra. Note that the solution becomes infinite when the effective frequency approaches the resting inertial frequency. It is shown in Appendix D that the limit of the solution in this case is the resonant solution one would expect when forcing a resting ocean at the inertial frequency. This solution confirms the fact that the inertially rotating winds force motions below the resting inertial frequency in the negative vorticity region, which then can become trapped.

One interesting consequence of the spatial structure of the solution is that the near-inertial kinetic energy and the work done by the wind on inertial motions vary significantly across the front during the 19 hour forcing period, Figure 5-6. The most energy is put into the regions where the forcing frequency matches the local inertial frequency, which is on the outskirts of the front, and also directly in the middle of the jet. As the ocean surface velocity begins to rotate in response to the wind forcing, it stays in phase with the forcing, and therefore the energy in the resonant region increases at all times. In the regions where the relative vorticity is important, less total energy is put into the system because the ocean oscillations don't rotate in phase with the forcing. 

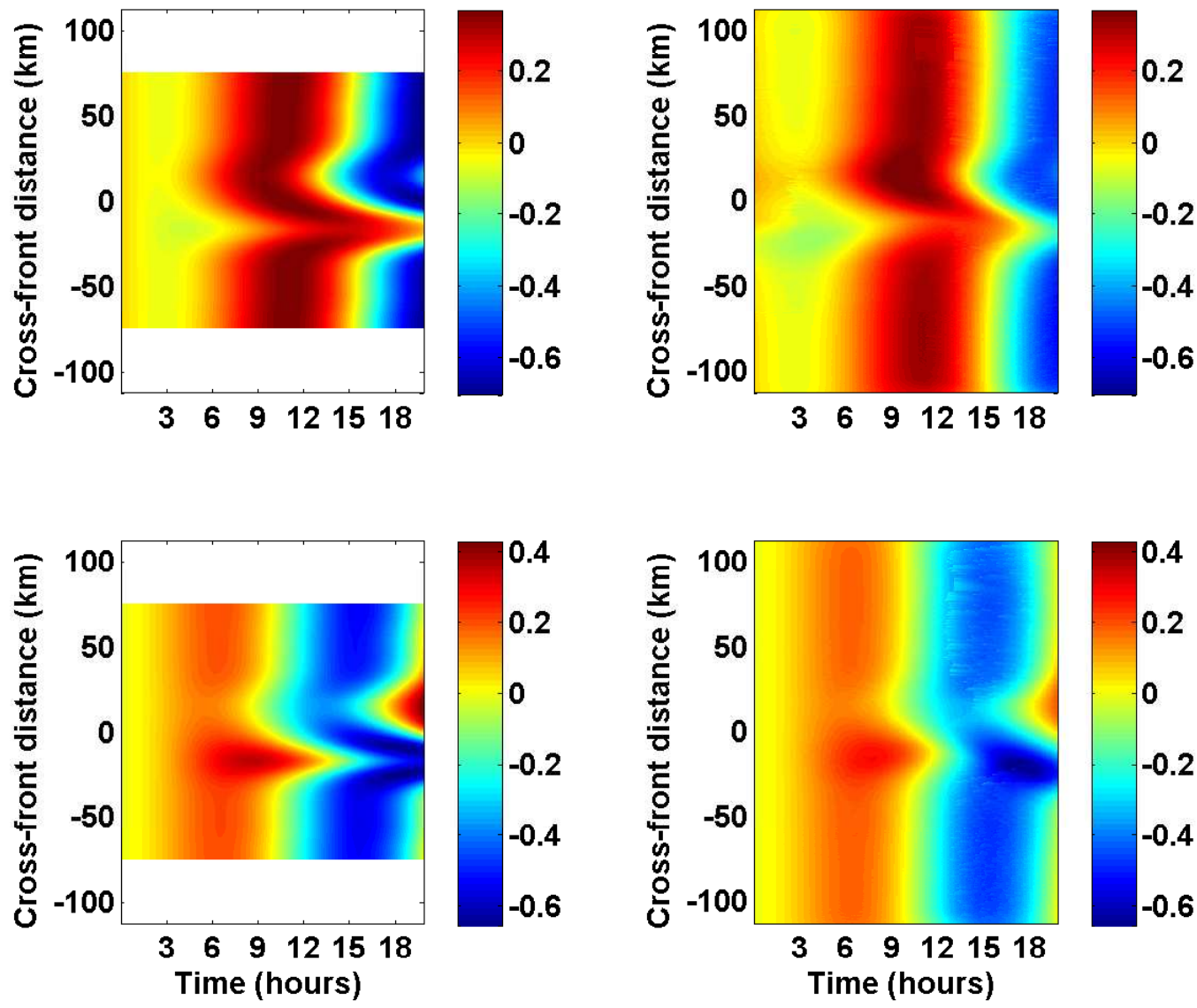

Figure 5-5: Zonal (top) and meridional (bottom) velocity $\left(m s^{-1}\right)$ during the wind forcing from both the analytical solution (left) and the model output (right).

\subsubsection{Decay of mixed layer near-inertial kinetic energy}

As discussed above, it has been shown that the presence of a strong relative vorticity gradient (regardless of the sign of the relative vorticity) results in a more rapid vertical propagation of near-inertial energy out of the mixed layer into the ocean interior. This occurs because the upper ocean structure imparts a shorter horizontal wavelength on the inertial motions than the forcing wavelength, and vertical group speed is inversely dependent on the horizontal wavelength (Pedlosky, 2003):

$$
c_{g z}=\frac{f^{2}-N^{2}}{\omega K^{4}} m k^{2}
$$



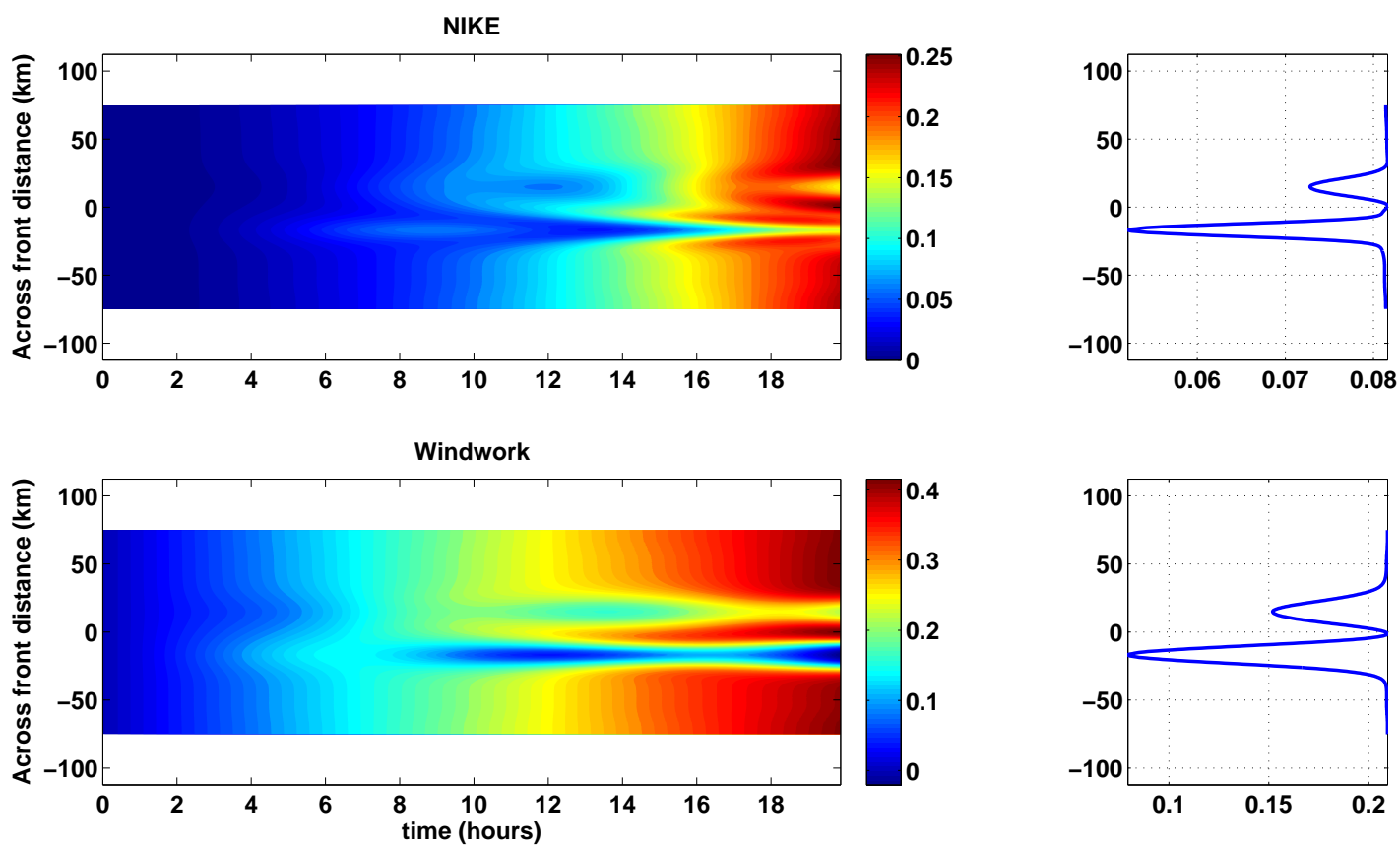

Figure 5-6: Near-inertial kinetic energy (top panel) $\left(\mathrm{m}^{2} \mathrm{~s}^{-2}\right)$ and wind work (bottom panel) $\left(\mathrm{Nms}^{-1}\right)$ from the analytical solution.

(where $c_{g z}$ is the vertical group speed, $\omega$ is the frequency of the wave, $K^{4}$ is the magnitude of the wavenumber vector, $m$ is the vertical wavenumber, and $k$ is the horizontal wavenumber). The rapid vertical propagation of near-inertial kinetic energy out of the mixed layer results in the relatively quick decay time for surface forced inertial oscillations in strong relative vorticity gradients. Through examination of the temporal and across-front evolution of the modeled surface near-inertial kinetic energy, it can be seen that the 3DPWP model captures the expected mixed layer decay behavior, Figure 5-7; the near-inertial kinetic energy clearly persists near the surface of the model domain for a longer time in the outskirts of the jet, and a shorter time in the jet (which is associated with the relative vorticity signal). Decay times were estimated as the time it took the near-inertial kinetic energy to drop from its maximum to five percent of that value, and show that the mixed layer oscillations in the center of the jet decay rapidly (in a few days), while the oscillations on the outskirts 


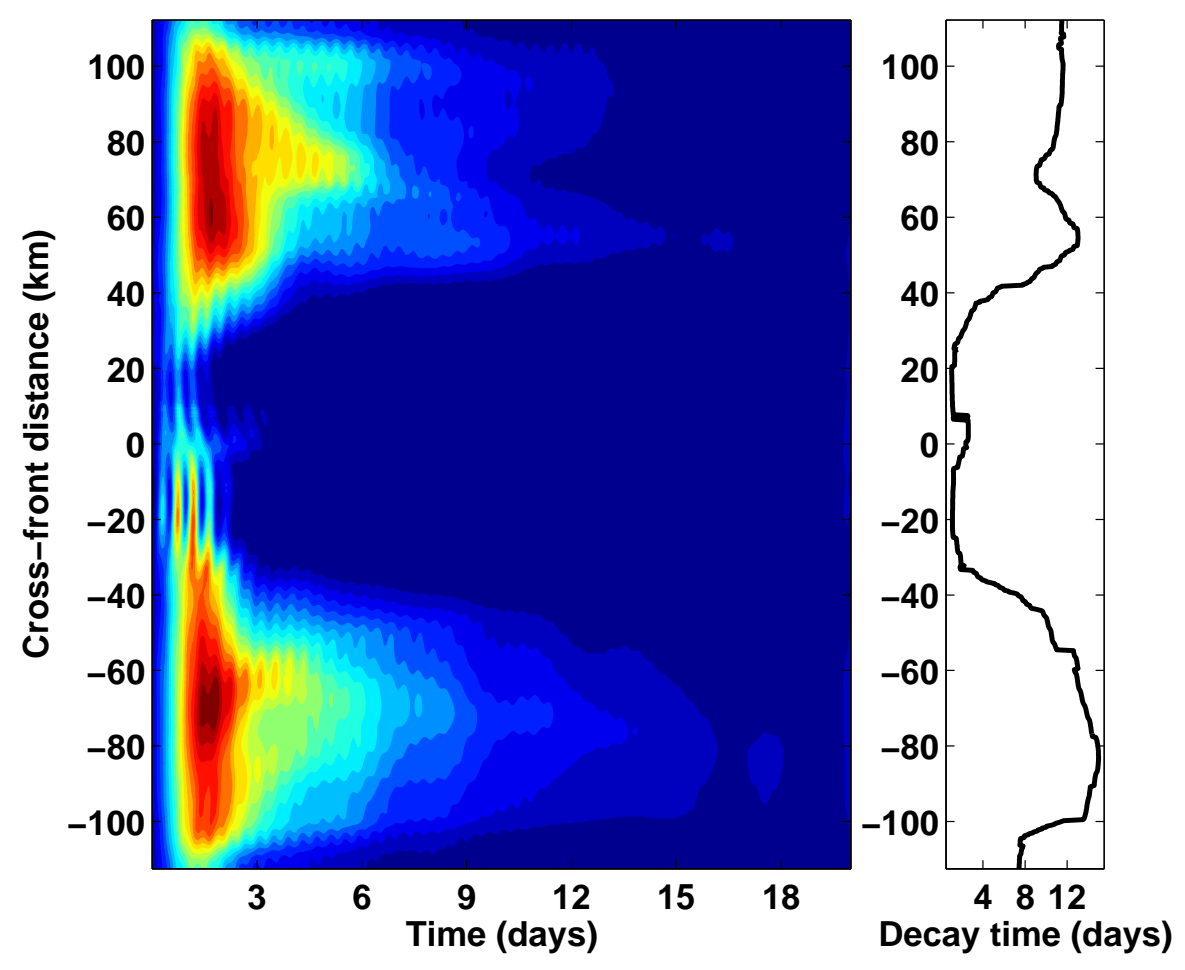

Figure 5-7: Cross front/time section of the surface near-inertial kinetic energy. The right panel shows the decay time for the near-inertial oscillations. 
of the jet linger for almost two weeks, Figure 5-7 (right panel). These decay times are slightly faster than expected for an $f$-plane simulation; in the absence of beta, the mixed layer near-inertial decay time according to linear theory is on the order of a month (in the absence of relative vorticity gradient) (Gill, 1984). However, the model does capture the correct pattern of decay behavior.

\subsubsection{Vertical wavelength and mixing}

The above analysis of the frequency response and mixed layer decay times indicate that the modeled behavior of the near-inertial oscillations in the mixed layer are consistent with previous studies. As the near-inertial energy propagates out of the mixed layer to the interior in the negative vorticity region, theoretically it is unable to propagate out into regions where the local frequency is above the effective frequency of the near-inertial wave. It has been suggested that as the vertical wavelength of the wave shrinks and the horizontal velocity amplitude grows as vertical critical layers are approached, the trapped energy is lost to mixing associated with the enhanced vertical shear (Kunze et al., 1995). In order to determine the evolution of the vertical scales of the wind-forced internal waves as they approach critical layers, vertical wavenumber spectra were calculated over a depth range which is consistent with the theoretical location of critical layers. Critical layers are located at the vertical depth where the intrinsic frequency of the wave matches the effective frequency of the background flow. The depth range of the critical layers for this model run was estimated by comparing the surface frequency response in the negative vorticity region with the initial variation of effective frequency with depth (estimated from the initial geostrophic velocity), and was found to be 200 to 1000 meters. Examination of the temporal evolution of the vertical wavenumber spectrum evaluated over the critical layer depth range in several sections across the front shows a clear, energetic transfer of energy from larger to smaller scales in the negative vorticity region, Figure 5-8. The regions to the north and south of the jet as well as the negative vorticity region show no noticable tranfer of energy to small vertical scales, and are much less energetic 


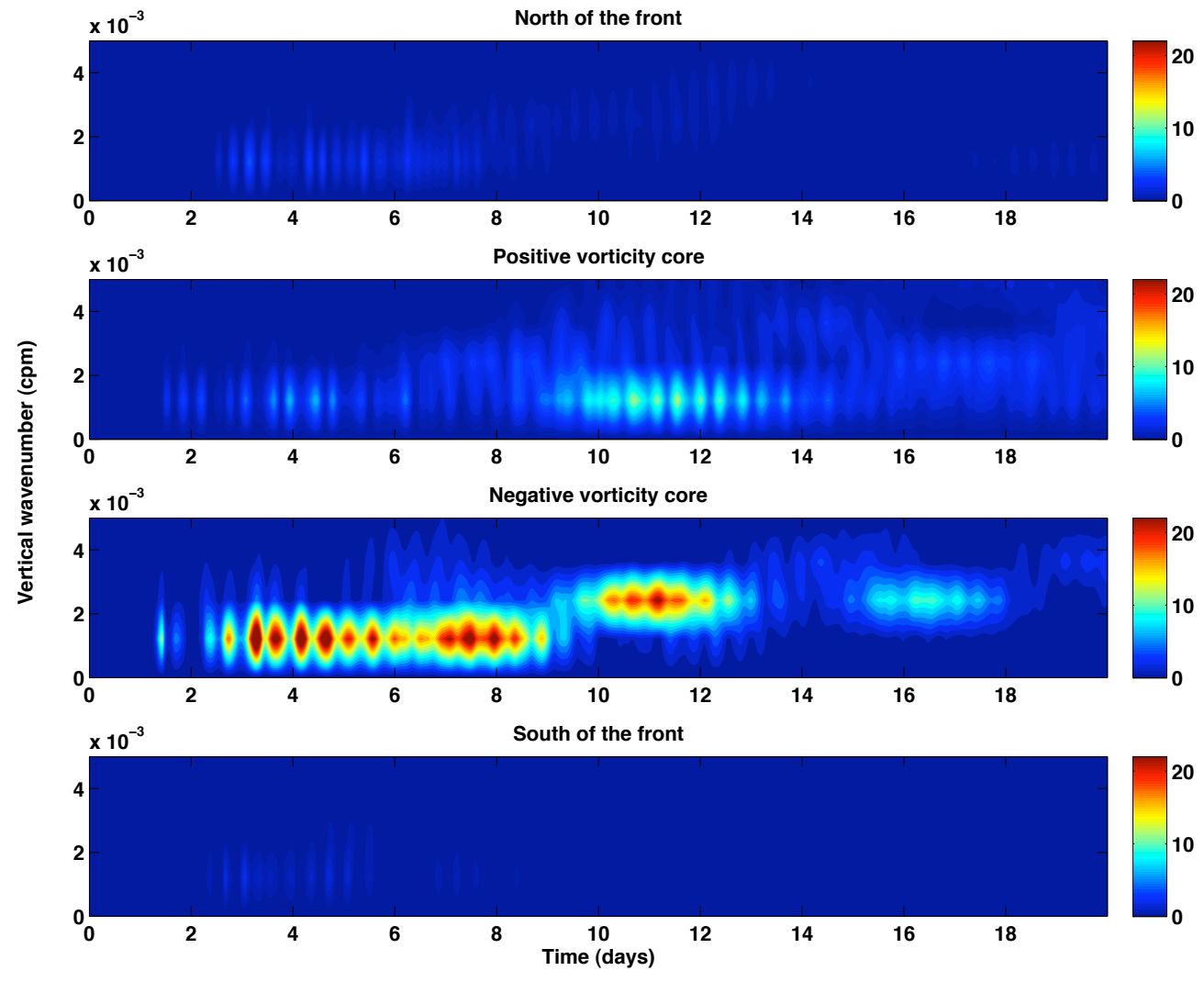

Figure 5-8: Time evolution of vertical wavenumber spectra $\left(\mathrm{m}^{2} / \mathrm{s}^{2} / \mathrm{cpm}\right)$ of the meridional velocity covering the depth range from 200 to 1000 meters. Top panel: region north of the front; middle top panel: positive vorticity core; middle bottom panel: negative vorticity core; bottom panel: region south of the front. 
than the negative vorticity region.

The modeled transfer of energy to smaller vertical scales is consistent with the picture that near-inertial energy trapped in the negative vorticity region is rapidly being transferred to smaller scales. This then leads to large vertical shears, and consequently shear-instability mixing. The spatial distribution of the modeled Richardson number mixing (both gradient and bulk) supports this scenario. By looking at a cross-front section of the number of time steps the model relieves shear instability over the course of the model run, it can be seen that there is a triangle of enhanced mixing in the negative vorticity region which is strikingly absent on the north side of the front, Figure 5-9. Given all the previous results, we suggest that this enhanced

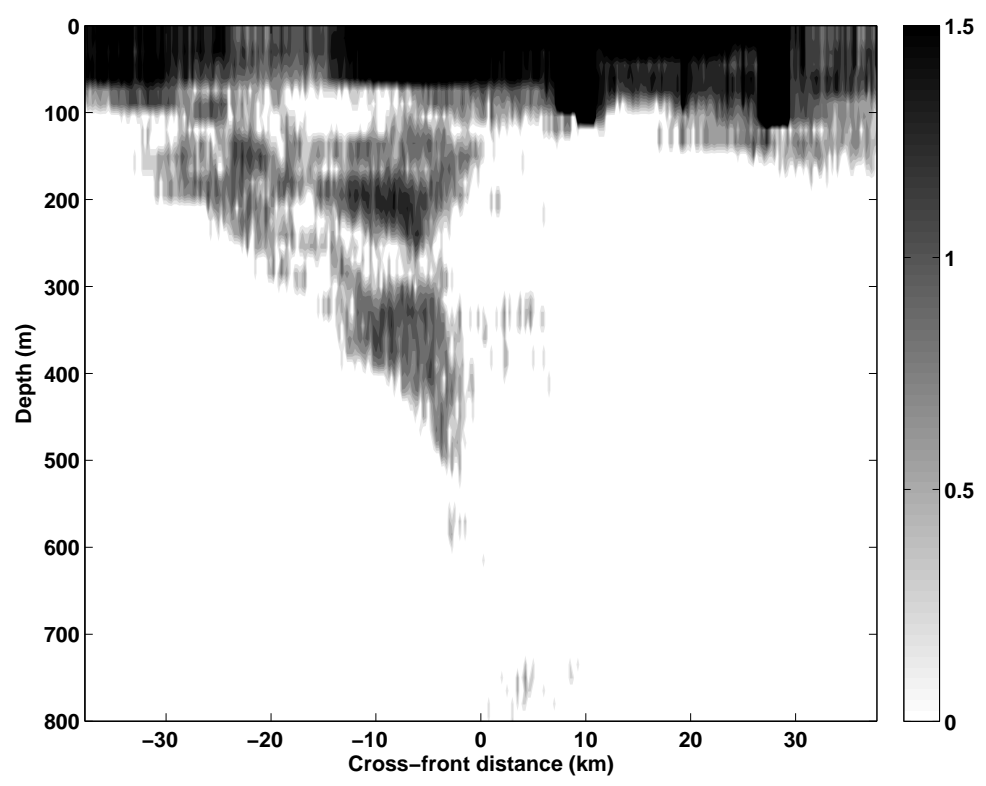

Figure 5-9: Cross-front/depth section of the logarithm of the number of times the Richardson number mixing loop is called. 
mixing is associated with the trapping of near-inertial internal waves. This shear instability mixing occurs in the southern core of the Gulf Stream, which is the region where EDW formation is thought to take place. A closer look at the dynamics of this enhanced mixing is presented in the following section.

\section{$5.4 \quad$ Near-inertial kinetic energy budget}

\subsubsection{Formulation}

A near-inertial kinetic energy budget was used to characterize and quantify the modeled mixing of trapped near-inertial waves. We begin with the momentum equations that the 3DPWP model implements:

$$
\begin{gathered}
\frac{\partial u}{\partial t}+\vec{u} \cdot \nabla u-f v=\frac{-1}{\rho_{0}} \frac{\partial F_{x}}{\partial z} \\
\frac{\partial v}{\partial t}+\vec{u} \cdot \nabla v+f u+\frac{1}{\rho_{0}} \frac{\partial p}{\partial y}=\frac{-1}{\rho_{0}} \frac{\partial F_{y}}{\partial z} .
\end{gathered}
$$

Here, we are using the convention that the $\mathrm{x}$ axis is the along-front direction, and the $\mathrm{y}$ axis is the across-front direction. Therefore, the $\mathrm{x}$ derivatives have been elimated from the momentum equations, as there is no variation in the along-front direction. The right hand side terms can be written as $\frac{\partial F_{x}}{\partial z}=\frac{\partial F_{x f}}{\partial z}+\frac{\partial F_{x s}}{\partial z}+\frac{\partial F_{x r}}{\partial z}$ and represent the changes in momentum which occur as a result of the wind stress application $\left(\frac{\partial F_{x f}}{\partial z}\right)$, static instability mixing $\left(\frac{\partial F_{x s}}{\partial z}\right)$ and Richardson number mixing $\left(\frac{\partial F_{x r}}{\partial z}\right)$. The momentum equations were filtered over a broad near-inertial band preserving motions with periods of 16 hours to 30 hours. This range was determined by studying the model output spectra. The filtering is implemented by taking the Fast Fourier Transform of the terms, setting all but the desired frequency components to zero, and taking the inverse Fast Fourier Transform.

$$
\frac{\partial u_{i}}{\partial t}+\langle\vec{u} \cdot \nabla u\rangle-f v_{i}=\left\langle\frac{-1}{\rho_{0}} \frac{\partial F_{x}}{\partial z}\right\rangle
$$




$$
\frac{\partial v_{i}}{\partial t}+\langle\vec{u} \cdot \nabla v\rangle+f u_{i}+\frac{1}{\rho_{0}} \frac{\partial p_{i}}{\partial y}=\left\langle\frac{-1}{\rho_{0}} \frac{\partial F_{y}}{\partial z}\right\rangle
$$

where \langle\rangle represent the near-inertial filtering operation. Equations 5.12 and 5.13 are then multiplied by $u_{i}$ and $v_{i}$ respectively and then added, yielding the kinetic energy equation.

$$
\frac{\partial K E_{i}}{\partial t}+u_{i}\langle\vec{u} \cdot \nabla u\rangle+v_{i}\langle\vec{u} \cdot \nabla v\rangle+\frac{v_{i}}{\rho_{0}} \frac{\partial p_{i}}{\partial y}=u_{i}\left\langle\frac{-1}{\rho_{0}} \frac{\partial F_{x}}{\partial z}\right\rangle+v_{i}\left\langle\frac{-1}{\rho_{0}} \frac{\partial F_{y}}{\partial z}\right\rangle
$$

This equation shows that the temporal evolution of the near-inertial kinetic energy can be altered by triple product term, work done by the pressure, wind forcing, and parameterized mixing. The triple product term represents advection of the nearinertial kinetic energy by the near-inertial flow as well as exchanges of kinetic energy between the near-inertial and other (higher and lower) frequency bands.

The 3DPWP model doesn't calculate the turbulent momentum flux $(F)$ directly; instead mixing occurs in response to critical bulk Richardson number (Richardson number estimated at the base of the mixed layer to be less than 0.6), critical gradient Richardson number (Richardson number estimated through the water column below the mixed layer to be less than 0.25 ), and static instability. In order to obtain the mixing and wind forcing terms for the kinetic energy budget, vertical profiles of the velocity components were saved before and after the model implemented mixing or

applied wind stress during each time step. Using these before and after profiles, the exact change in momentum due to each of the mixing schemes and the wind forcing can be determined. The resulting time series of momentum changes were then filtered over the near-inertial band as described above for all the budget terms.

\subsubsection{Kinetic energy budget results}

In order to highlight spatial variability of near-inertial behavior, the terms in the kinetic energy budget were integrated over the positive and negative vorticity cores of the background flow field. The positive (negative) vorticity core was defined as 
the region where the relative vorticity was greater (less) than $(-) 2 x 10^{-5}\left(s^{-1}\right)$. Nearinertial kinetic energy can be found at greater depths in the negative vorticity region than in the positive vorticity region, Figure 5-10. Deeper penetration of the near-
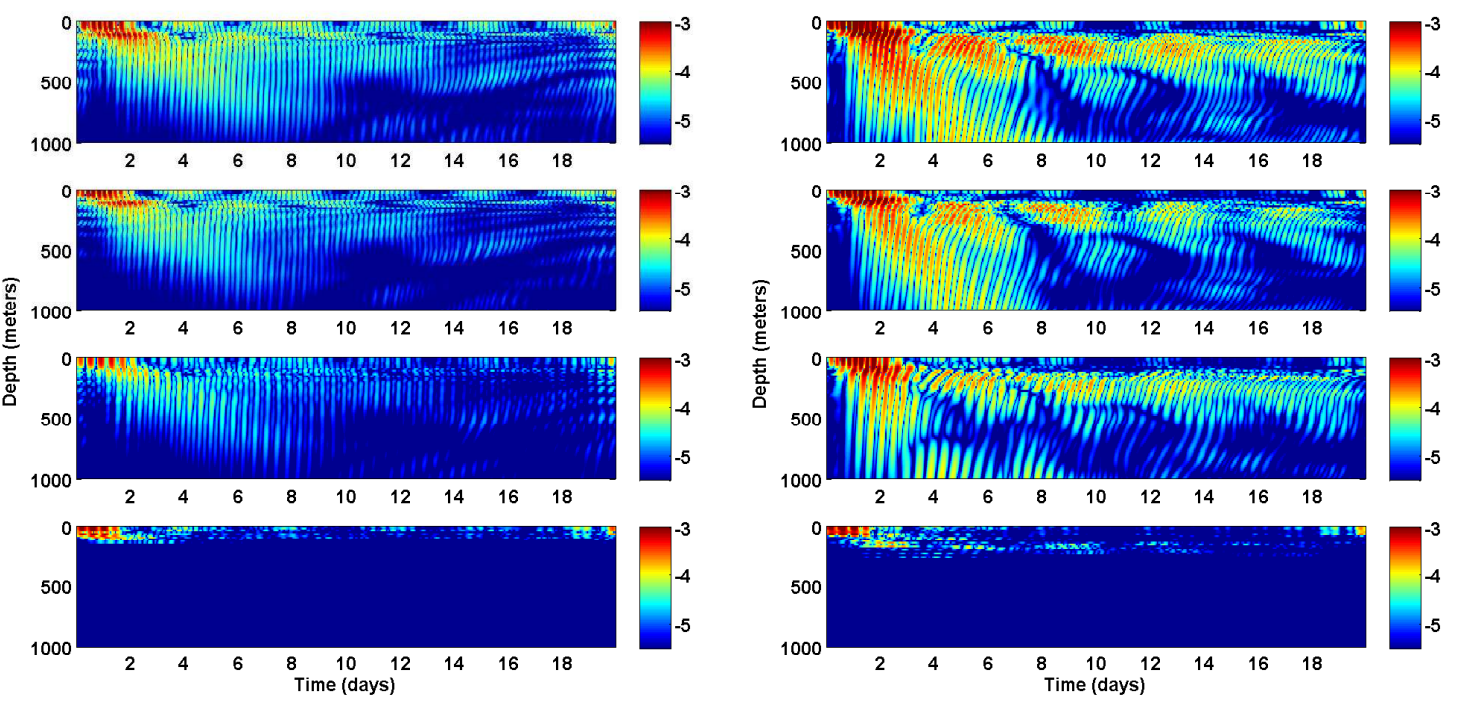

Figure 5-10: Components of the near-inertial kinetic energy budget integrated over the positive (left) and negative (right) vorticity cores. Budget terms shown are temporal evolution of kinetic energy (top panel), wave-wave interaction (second panel), pressure work (third panel), and mixing (bottom panel).

inertial kinetic energy in the negative vorticity region is consistent with previous model studies (Danioux et al., 2008; Lee and Niiler, 1998). In addition, near-inertial kinetic energy persists for longer in the negative vorticity region, particularly in the depth range below the mixed layer and above 500 meters depth. Despite the fact that the parameterized mixing can be at times as important as the other two terms in the kinetic energy budget, it is clear that the overall near-inertial kinetic energy distribution is controlled by wave-wave interaction and pressure work. Physically, these two terms represent both the spatial transfer of near-inertial kinetic energy by the near-inertial motions themselves and transfers of kinetic energy in and out of the near-inertial frequency band (triple product term) as well as the radiation of near-inertial energy by the internal waves (pressure work term). It is perhaps not 
surprising that these two terms dominate the near-inertial kinetic energy budget (even in the negative vorticity region) as the surface wind forcing generates near-inertial energy across the whole domain, and motions with an intrinsic frequency above the background effective frequency are free to propagate horizontally.

Side by side comparison of the mixing contribution in the two displayed regions demonstrates clear differences. There is a deeper layer of enhanced mixing, spanning approximately 100 to 300 meters depth, in the negative vorticity side which is absent on the positive side. This layer corresponds to the increased number of Richardson number mixing instances described above, and could be a critical layer where the downward propagating near-inertial energy stalls and undergoes shear instability and mixing. This shear-driven mixing is sporadic but persists throughout the first eighteen days of the twenty day model run while weakening in intensity over the course of the model run.

\subsection{Implications for EDW formation}

The above work shows that wind forcing, in this case inertially rotating winds, can result in near-inertial motions which become trapped in the negative vorticity region of the Gulf Stream. This trapping then leads to enhanced vertical shear and ultimately dissipation of the trapped near-inertial motions through shear-driven mixing. Given the CLIMODE observations described in Chapter 2 of this thesis which detected the presence of motions at a frequency below the local resting frequency in the south core of the Gulf Stream, it is plausible that this trapping mechanism can lead to enhanced mixing in the EDW formation region during the passage of wintertime storms. This mixing can affect EDW formation by altering the background stratification. We hypothesize that the enhanced mixing could potentially lead to deep mixed layers in the EDW formation region, therefore contributing directly to EDW formation. Another possibility is that the mixing preconditions the water column for buoyancy forced deep convective mixing by creating a patch of weak vertical buoyancy gradient 
below the mixed layer.

Examination of the evolution of the buoyancy frequency below the mixed layer indeed suggests that there is a weakening of the stratification at approximately two hundred to three hundred meters depth in the negative vorticity region associated with the near-inertial trapped wave mixing, Figure 5-11. This patch of weakly stratified
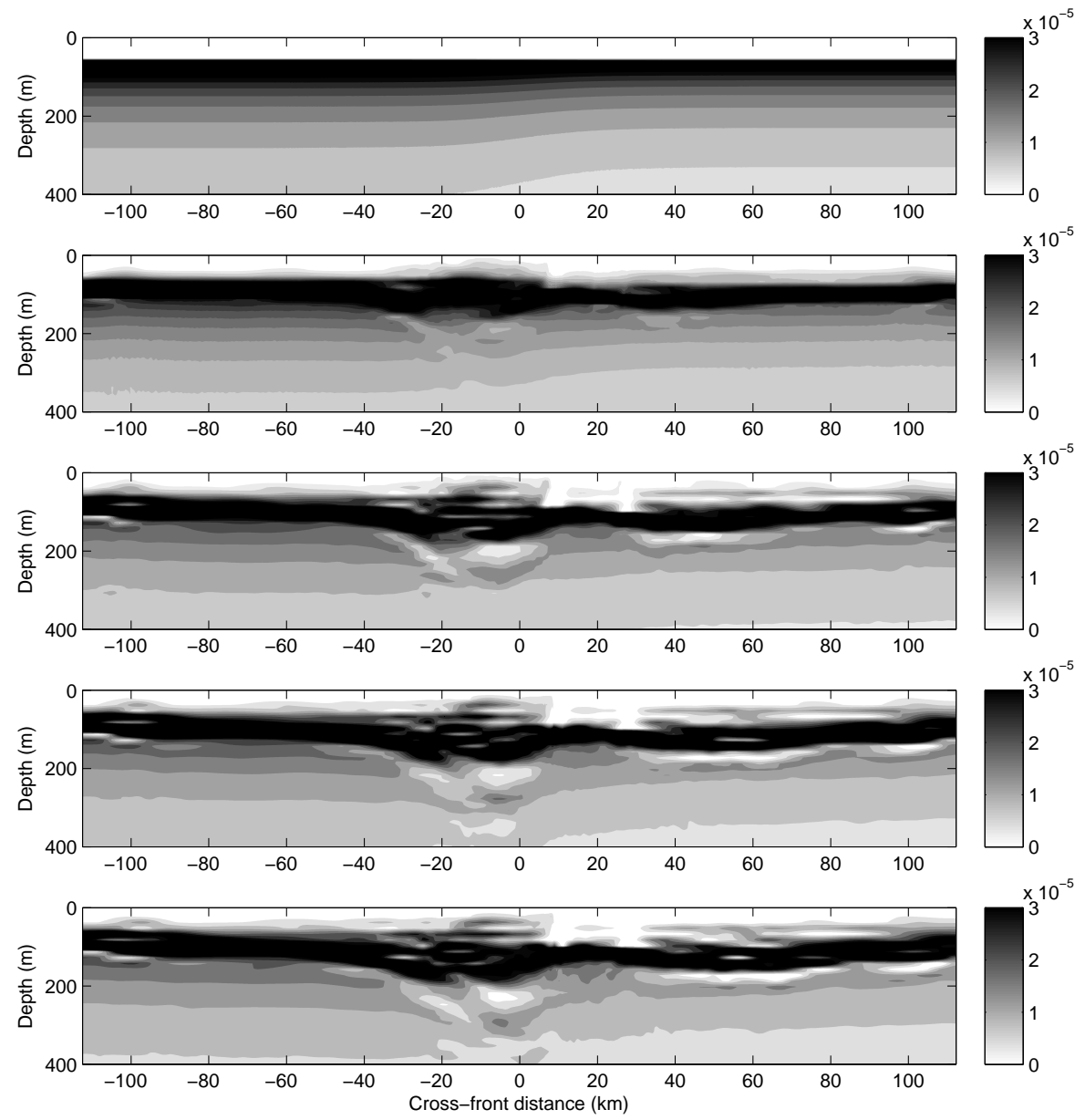

Figure 5-11: Initial buoyancy frequency squared $\left(s^{-2}\right)$ distribution (top) and successive five day time mean buoyancy frequency squared distribution (bottom four panels).

water appears within five to ten days after the wind forcing, persists throughout the remainder of the twenty day model run, and is noticeably absent on the positive 
vorticity side. Restratification of the mixed layer in the negative vorticity region is also visible, and occurs within the first four days after the wind forcing.

Restratification of the mixed layer in the negative vorticity region is a surprising result in light of the enhanced mixing in that region, and suggests that mixed layer processes occurring during the course of the model run can act to impede the formation of EDW. Further analysis was therefore carried out in an attempt to address the cause of these near-surface density changes. To begin, an identical model run with no wind forcing was executed to assess the possibility that the modeled restratification results solely from the adjustment of the frontal system over time. There is restratification in the mixed layer in the unforced run suggesting that the density changes can be accounted for by frontal slumping (which could be due to numerical diffusion, and therefore a byproduct of the choice of model and parameters), Figure 5-12. A simple calculation was carried out in order to qualitatively assess how much of the observed change in mixed layer stratification in the negative vorticity region in the wind-forced run can be accounted for by the restratification observed in the unforced run. For both the forced and the unforced run, the buoyancy frequency was integrated vertically over the depth of the initial mixed layer (60 meters) and horizontally over the negative vorticity region. Comparing the initial value to the integrated buoyancy frequency at the end of the wind forcing, it was found that $60 \%$ of the change in the stratification is due to the adjustment seen in the unforced run.

Thomas and Ferrari (2008) show that wind forcing can also alter the stratification in the mixed layer, and that the contribution from the wind to changes in stratification can be calculated using a term from the potential vorticity budget as follows:

$$
J_{z}^{F}=\left(\nabla_{h} b \times F_{h}\right) \cdot \hat{z}
$$

where $J_{z}^{F}$ is the vertical component of the frictional potential vorticity flux, $b$ is the buoyancy, and the expression $F_{h}=\frac{\vec{\tau}}{\rho_{0} h}$ is used to specify the wind component of the frictional PV flux. Using the model buoyancy field and the wind forcing, the 

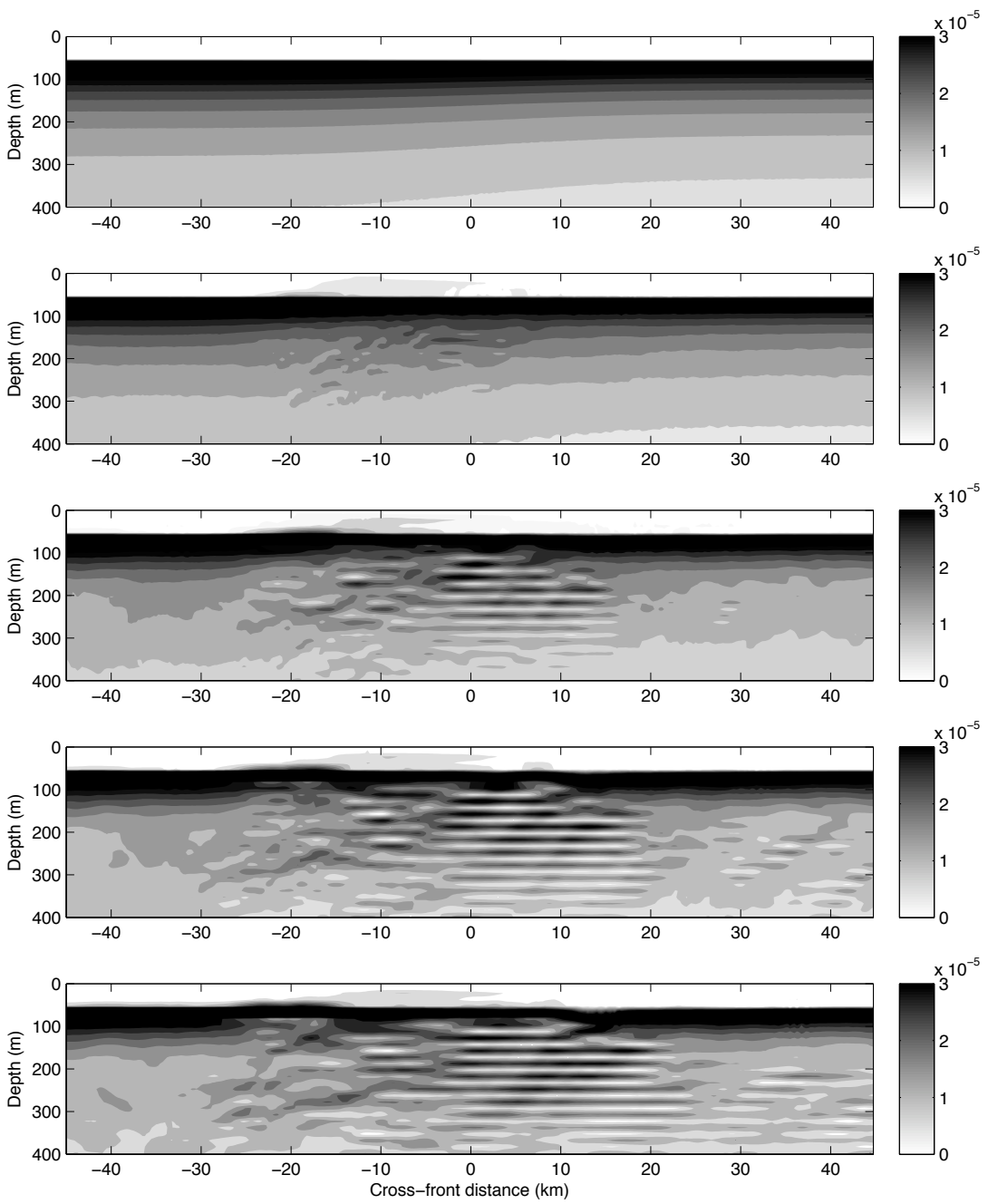

Figure 5-12: Initial buoyancy frequency squared $\left(s^{-2}\right)$ distribution (top) and successive five day time mean buoyancy frequency squared distribution (bottom four panels).

temporal change in stratification due to the wind-forced frictional PV input is found to account for only $20 \%$ of the total. Therefore, although the wind does contribute to the restratification in the mixed layer in the negative vorticity region, the dominant cause is the adjustment of the model over time which is independent of forcing and potentially an artifact of the model itself.

Although there is evidence of near-inertial wave trapping and mixing in the model 
Gulf Stream of the simulation described in this chapter, the buoyancy frequency in the associated weakened stratification region (200 to 300 meters depth) only decreased by $1 \%$, and therefore we conclude that, for this particular model configuration and forcing, this mechanism cannot substantially enhance EDW formation, as hypothesized. And, it was found that the inertially rotating wind forcing can potentially contribute to restratification in the mixed layer in the negative vorticity region, which will act to impede EDW formation. It should be kept in mind, however, that other wind forcings might produce very different results. It is also important to remember that an isolated wind event in which the upper ocean is allowed to evolve for twenty days with no subsequent forcing is very unrealistic for the wintertime Gulf Stream environment. More typically, a rapid succession of storms are constantly imparting wind energy to the ocean surface, and it seems possible that when this is taken into account, different results could be obtained. Another possible extension of this work is a model run which includes both wind and buoyancy forcing; the inclusion of strong convective mixing in the upper ocean may change the dynamics of the wind forced response and therefore the resulting upper ocean stratification. 


\section{Chapter 6}

\section{Concluding remarks}

\subsection{Discussion}

Results presented in Chapter 2 of this thesis provided insight about the temporal and vertical distribution of near-inertial kinetic energy as observed from a long-term mooring in the North Atlantic Ocean. After developing a simple filtering technique to isolate the near-inertial kinetic energy, both seasonal wintertime enhancement and a surface intensification of the near-inertial kinetic energy were described. The chief exception to this was an event characterized by significant near-inertial energy found deep in the water column, which is thought to be associated with the passing of a Gulf Stream warm core ring. Finally, a depth integrated model consisting of a wind forcing term and a dissipation paramaterization was implemented, and shown to capture the observed seasonal cycle of the near-inertial kinetic energy.

Long term records of near-inertial motions with high vertical resolution are not prevalent, probably because of the high sampling frequency required, and thus the observations described in Chapter 2 are valuable. Not only do they capture the seasonal cycle of near-inertial motions, but they also give a glimpse of the underlying interannual variability, which has been linked here to the variability in wind forcing. The patterns of surface intensification and winter enhancement are in agreement with the global patterns of near-inertial energy variability presented by Alford and 
Whitmont (2007). To first order, the depth-integrated model shows that the nearinertial energy imparted by the wind is balanced by localized dissipation. This result suggests that the pattern of the wind forced component of the dissipation required to close the abyssal circulation, as described by Munk and Wunsch (1998), could be related to the distribution of near-inertial wind work.

Chapter 4 represents a shift in the focus of this thesis from surface wind forcing to surface buoyancy forcing. Upper ocean temperature and velocity measurements are presented from two drifting profiling floats (EM-APEX) and a drifting spar meteorological buoy with a tethered upper ocean profiler (ASIS-FILIS). Deployments of these instruments were made as part of the CLIMODE program, which was designed to capture the extreme surface forcing associated with the passage of wintertime cold air systems over the Gulf Stream region. Because of the quasi-Lagrangian nature of the intruments, the analysis was expected to show a strong correlation between the surface heat flux and the evolution of the upper ocean heat content. However, the strong vertical current shear of the Gulf Stream in combination with the profiling nature of the instruments resulted in the dominance of the mixed layer heat balance by relative advective terms. There are multiple examples of heat budget studies from drifting platforms, and there seems to be mixed success amongst these studies at achieving the goal of reducing advective influences.

Previous observational and model studies had mixed conclusions about the relative importance of terms in the Gulf Stream heat balance. Kelly and Qui (1995) suggested advection dominated the heat balance, while Xue et al. (1994) observed a nearly onedimensional response of the upper ocean to a winter storm passage over the Gulf Stream off the coast of North Carolina. The results presented in Chapter 4 support the former hypothesis; if advection terms dominated in the quasi-Lagrangian mixed layer heat balance then they would certainly be of first order importance in a Eulerian framework. It is possible that differences between the east/west orientation of the Gulf Stream in this study and the north/south orientation of the Gulf Stream in the Xue et al. (1994) work could account for these discrepancies. 
The drifting profiling float records used to evaluate the heat balance in Chapter 4 were also analyzed for the presence of near-inertial motions in Chapter 3. Not only were there energetic near-inertial motions in both float records in the Gulf Stream region, but the frequency of those oscillations was found to be depressed below the resting inertial frequency, suggesting the possibility of near-inertial wave trapping below the mixed layer. Because the drifting profiling floats deployments were of short duration and consisted of sporadic spatial information, the modeling study presented in Chapter 5 was carried out in order to examine the potential impacts of the trapping mechanism on the stratification. Inertially rotating wind forcing resulted in an energetic cascade of energy to small vertical wavenumbers, associated enhanced mixing, and therefore weakened stratification in the negative vorticity region below the mixed layer. However, frontal slumping and wind forcing in the surface layer led to restratification of the mixed layer, providing a competing effect which can counteract the effects of enhanced mixing on stratification.

Previous studies (both observational and theoretical) had described the rapid decay of near-inertial mixed layer oscillations in the presence of a background relative vorticity gradient (Meurs, 1998; Balmforth and Young, 1999). In addition, prior model work had shown that near-inertial kinetic energy penetrated deeper in regions of negative vorticity than in positive vorticity (Danioux et al., 2008). Observations of a warm core Gulf Stream ring had suggested that the ultimate sink for energy trapped in negative vorticity regions was dissipation as a direct result of vertical shear driven mixing (Kunze et al., 1995). The model study presented in Chapter 5 contributes to this literature by not only capturing the rapid mixed layer decay in the gradient relative vorticity region, but also by describing the characteristics of the trapped near-inertial energy and quantifying the shear driven mixing that results. In addition, the model results suggest that the trapping mechanism could potentially have an impact on Eighteen Degree Water formation by preconditioning the water column for the surface buoyancy flux driven convective mixing, with the caveat that mixed layer processes can potentially restratify the upper ocean and counteract this 
effect.

\subsection{Future work}

There is still much work to be done in order to obtain a complete understanding of the pathway of wind-forced near-inertial energy. In addition to the depth-integrated nearinertial energy model developed for this thesis, the observations presented in Chapter 2 could be examined more closely to try to understand the depth dependence of the kinetic energy budget. Additional observations which allowed the estimation of one or more components of the horizontal energy flux would be beneficial for that kind of analysis. And, of course budgets of near-inertial kinetic energy would benefit greatly from measurements of both surface wind stress and surface ocean velocity, which will allow for direct calculation of the work done by the wind on near-inertial motions.

In addition to the seasonal variability in the near-inertial kinetic energy signal, the work presented in this thesis supports the notion that near-inertial waves can be trapped in negative vorticity regions and dissipate locally, which can disrupt the pathway of wind-forced energy. We find here evidence of enhanced mixing in these regions, but the kinetic energy budget suggests that this sink of energy is not a large one compared with advection and radiation of near-inertial waves. It's still not clear how important this trapping mechanism is from a global point of view. Also, the model work presented here examines an isolated case of wind forcing in which the upper ocean is allowed to evolve unforced for an unrealistically long time. Clearly, the effects of repeated wind forcing, as well as different forms of wind forcing need to be taken into account. The strong buoyancy forcing which accompanies the wintertime storms and the resulting convective mixing may also interfere with the trapping mechanism, so the combined wind/buoyancy forcing problem must be addressed as well.

The observations of buoyancy forcing and the resulting upper ocean heat balance presented in Chapter 4 showed that the advective terms resulting from the profiling 
scheme of the floats dominated. This work suggests that the measurement of a Lagrangian heat balance in the high vertical shear regime of the Gulf Stream cannot be carried out successfully with profiling floats. In order to measure a Eulerian heat budget in this region, there would need to be instruments spaced around a central observation system to capture the components of the advection term, which will clearly dominate the budget, even in the presence of the strong wintertime surface heat flux. In addition, it would be of interest to perform similar heat budget analyses in both the north/south region of the Gulf Stream as well as the east/west section to try to understand if the upper ocean heat content response to surface forcing is different between these two scenarios, and if it is, to explain why. 


\section{Appendix A}

\section{A linear combination near-inertial}

\section{filter}

The MMP burst sampling scheme was exploited to calculate the near-inertial velocity as a linear combination of the burst velocity anomaly profiles as in equations 2.1 and 2.2. This scheme provides one estimate of the near-inertial velocity profile for each burst, and takes advantage of the fact that the ratio of the inertial period to the M2 tidal period at this latitude is $\sim 1.5$ to filter out M2 tidal energy. The calculation of near-inertial velocity with this method is sensitive to the phase of the near-inertial motions that have been sampled. However, because near-inertial motions are very nearly circularly polarized, and the velocity components are ninety degrees out of phase, calculation of the near-inertial kinetic energy as in equation 2.3 is only weakly dependent on the phase.

In order to quantify the efficacy of this near-inertial filtering scheme, current meter data from two Line $\mathrm{W}$ moorings were examined. The W2 and W4 moorings were located at $\left(39.2^{\circ} \mathrm{N}, 69.4^{\circ} \mathrm{W}\right)$ and $\left(38.4^{\circ} \mathrm{N}, 68.9^{\circ} \mathrm{W}\right)$, respectively, and both supported Vector Averaging Current Meters at 1000 meters depth that recorded at half-hour temporal resolution. Data taken from the time period spanning April 2004 to April 2006 were subsampled at half inertial period increments (9.5 hours) in order to mimic the MMP sampling scheme. The linear combination inertial filtering scheme was 
then applied to the subsampled current meter records to obtain near-inertial zonal and meridional velocities, and the near-inertial kinetic energy was calculated using equation 2.3. The full current meter records were also filtered using a Butterworth filter with a pass window of $4.5 \times 10^{-2}$ to $6 x 10^{-2} c p h$ (which corresponds to $.85 f$ to $1.15 f$ at the latitude of the moorings). A near-inertial kinetic energy time series was calculated for these filtered velocity records, and compared to the subsampled filtered kinetic energy, Figure A-1. It can be seen that the linear combination filtering

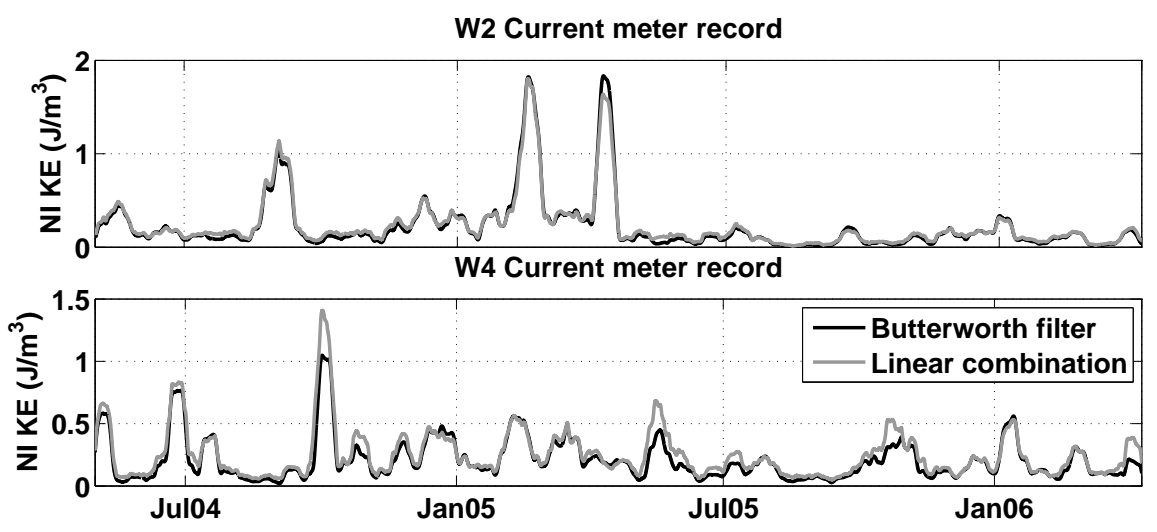

Figure A-1: Comparison of the subsampling inertial-filter technique with a Butterworth inertial-filter technique applied to Line W VACM records. The records were averaged over an 11 day period for comparison purposes.

technique accurately captures the magnitude and basic temporal evolution of the near-inertial kinetic energy. The frequency response of the filter was also estimated by comparing the kinetic energy spectrum of the full W4 current meter record and the kinetic energy spectrum of the same current meter record with the linear combination filter applied, Figure A-2. The linear combination filter passed 98 percent of the energy in the near-inertial frequency band, and just 3 percent of the energy in the semi-diurnal tidal band (defined here as $7-9 x 10^{-2} c p h$ ). Equations 2.1,2.2, and 2.3 were therefore applied to the MMP measurements to estimate near-inertial kinetic energy time series. 


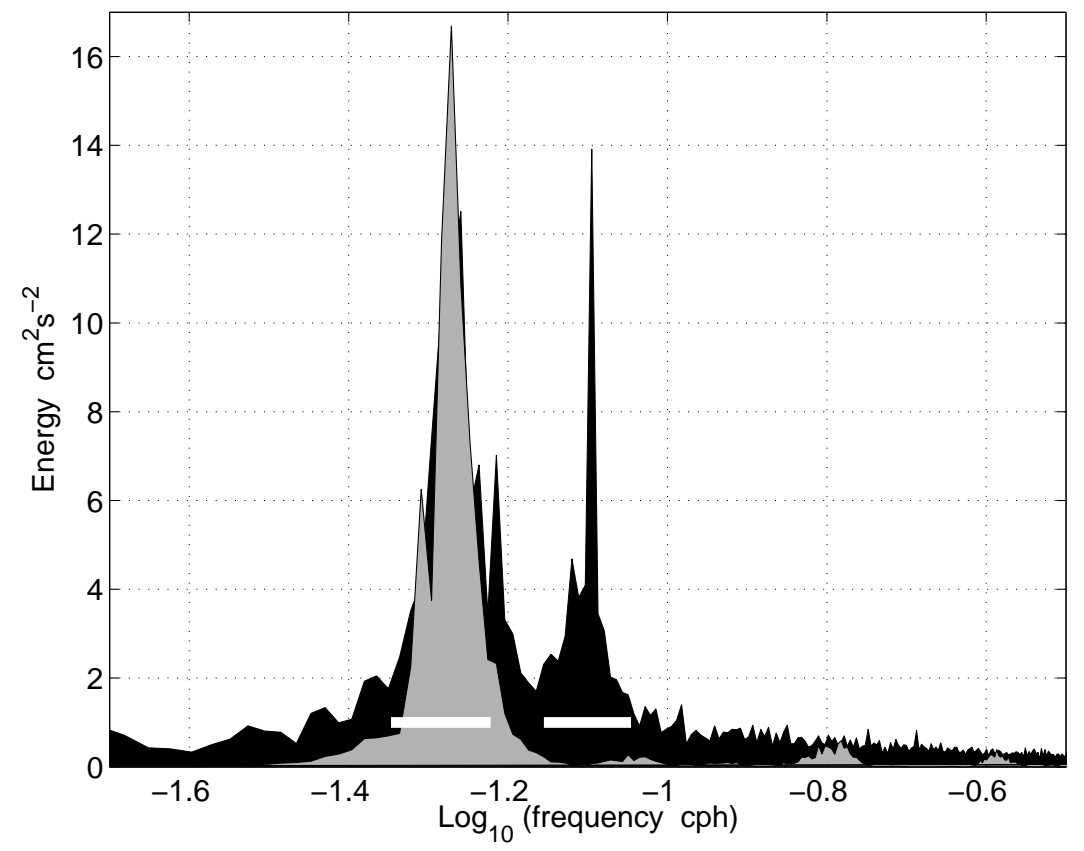

Figure A-2: Semilog plot of the kinetic energy spectrum of the full W4 current meter record (black) and the near-inertial kinetic energy estimated using the linear combination filter (gray). The white horizontal lines represent the extent of the near-inertial (left) and semi-diurnal (right) frequency bands. The spectra represent the average of 25 periodograms estimated from successive 28-day segments of the current meter record. 


\section{Appendix B}

\section{Estimation of the wind work on near-inertial motions}

For this study, the wind work on inertial motions was calculated as the dot product of the near-inertial surface currents with the near-inertial wind stress. Studies which have used a mixed layer kinetic energy budget to study wind forcing often use a different formula to estimate the wind work in which the wind work is dependent on the time derivative of the wind stress (D'Asaro, 1985; Plueddemann and Farrar, 2006). Analysis done for this study demonstrated that the differences between the two formulations are small and do not affect the conclusions of this paper.

Meteorological surface data were acquired from National Data Buoy Center (NDBC) Buoy \#44004 (www.ndbc.noaa.gov), which is located at $38.5^{\circ} \mathrm{N}$ and $70.4^{\circ} \mathrm{W}$, approximately 116 kilometers from the MMP moorings, Figure 2-2. There was a period of time when the buoy data were unavailable; during this interval, which totaled approximately 15 months of the 5.5 year analysis period, NCEP/NCAR Reanalysis data were interpolated to the NDBC buoy site and used in a similar fashion as the NDBC data. Comparison between the NDBC buoy data and NCEP/NCAR Reanalysis data at times when they were both available showed them to be in reasonable agreement.

Because of the distance between the NDBC buoy and MMP mooring locations, as well as the fact that the MMP moorings did not sample the surface mixed layer 
currents continuously, the PWP model was invoked to estimate the mixed layer nearinertial currents. Hourly sampled NDBC buoy (or NCEP/NCAR fields when the buoy was not operating) air temperature, wind direction and speed, atmospheric pressure, dewpoint temperature, and sea surface temperature were used in order to estimate a wind stress time series and the buoyancy forcing time series needed to drive the PWP model. The COARE bulk air-sea flux algorithm was utilized to estimate the wind stress and sensible and latent heat fluxes from the spliced NDBC and NCEP/NCAR Reanalysis data (Fairall et al., 1996). Longwave and solar heat fluxes were obtained strictly from the NCEP/NCAR Reanalysis data. The freshwater flux was neglected for the PWP runs.

Because PWP is a one dimensional mixed layer model and does not include terms such as advection, etc, it is necessary to reinitialize it occasionally in order to ensure that the model stratification remains close to the observed. Most of the wind energy input into inertial motions occurs early (in a fraction of an inertial period) within intense wind events. Between such events, the phase relationship between surface currents and the wind tends to be random (incurring little net energy input). With this in mind, a reinitialization scheme based on the wind stress magnitude was implemented. The model was reinitialized approximately one inertial period (19 hours) after the peak of strong wind events in which the wind stress magnitude surpassed a set threshold. Model reinitialization involved resetting density to the observed density profile, and the model velocity profiles to zero. The reinitialization scheme was run for two wind stress thresholds: $0.4 \mathrm{Nm}^{-2}$ and $0.6 \mathrm{Nm}^{-2}$, Figure B-1. The mean wintertime wind stress calculated from NDBC buoy and NCEP/NCAR Reanalysis data was $0.24 \mathrm{Nm}^{-2}$, with wintertime wind stress peaks reaching $1 \mathrm{Nm}^{-2}$.

In order to investigate the sensitivity of the wind work results to the PWP reinitialization scheme used, two additional PWP model runs were carried out. The first involved reinitializing the model on a monthly basis, with no dependence on wind stress, and the second initialized the model before strong $\left(>0.6 \mathrm{Nm}^{-2}\right)$ wind events (as opposed to after). It was found that the wind work time series calculated from 


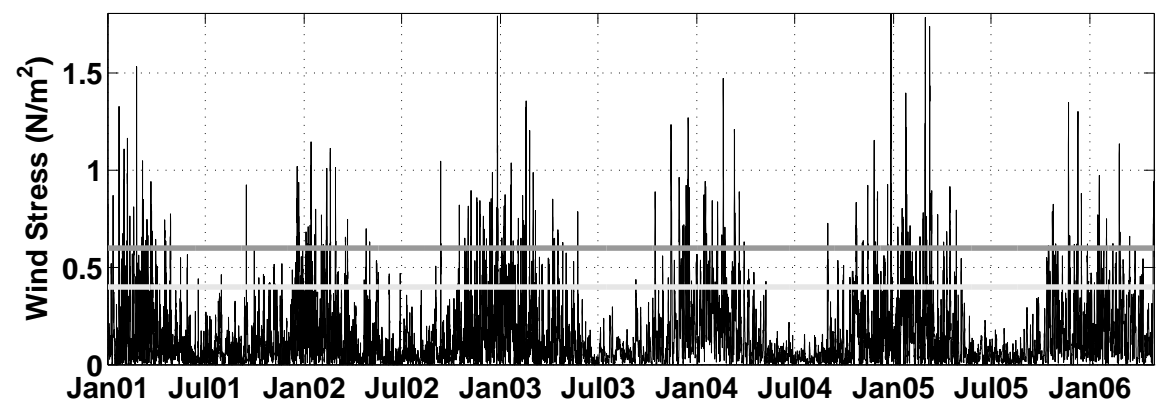

Figure B-1: Time series of the wind stress calculated from the spliced NBDC buoy and NCEP/NCAR Reanalysis data. The light gray line represents the $0.4 \mathrm{Nm}^{-2}$ reinitialization criteria, and the dark gray line represents the $0.6 \mathrm{Nm}^{-2}$ reinitialization criteria used for the PWP model runs.

all four of these model runs are similar in character, with the wind-stress-criteriabased model runs being comparable in magnitude as well. The monthly reinitialized model run results in an estimate for the time-averaged wind work which is smaller than that predicted from the other model runs by about 25 percent. Therefore, it was concluded that the results were not particularly sensitive to the reinitialization scheme, and ultimately the PWP run that was reinitialized after major wind events with a wind stress threshold of $0.6 \mathrm{Nm}^{-2}$ was adopted.

In addition to wind stress and buoyancy flux time series, the PWP model requires density profile data for the reinitializations, which came from the MMP temperature and salinity data. Because the MMP measurements didn't span the whole water column, it was necessary to extend the observed density profiles to the surface. Some of the MMP density profiles (particularly in winter) reached into the surface mixed layer; in these instances the temperature and salinity were extrapolated upwards to the surface as constant values. Whenever the MMP did not reach into the mixed layer, NDBC SST data were used to interpolate the temperature profile by linearly 
extrapolating the MMP temperature profile up from the shallowest sampled levels to the observed SST value, and assuming constant values above. Mixed layer depth was then calculated from the interpolated temperature profile. The MMP salinity profile was linearly extrapolated up to the inferred mixed layer depth and assumed constant above, thus allowing calculation of the density. If at the time of a reinitialization the SST was unknown and the MMP measurements didn't reach into the mixed layer, the current PWP model density profile was retained. The PWP model was run with an hourly time step spanning the period from fall 2001 to spring 2006. The model derived surface velocity and wind stress were subsequently filtered about the inertial frequency using a running boxcar technique with a pass window of $.5 f$ to $2 f$ before calculating the wind energy input into inertial motions using 2.6.

The PWP model runs were carried out with a linear drag parameterization as in Plueddemann and Farrar (2006). This drag term represents exchanges of momentum and energy between the base of the modeled layer and the ocean below beyond those associated with mixing in the transition layer. The lack of surface velocity observations in this region precluded attempts to tune the damping coefficient, $r$, to match the PWP surface velocities to the observations. Therefore a set of varying $r$ values were tested to determine the kinetic energy model's sensitivity to the damping coefficient.

The wind work input into inertial motions was evaluated in the form of a time integral of the wind work term $\left(P_{w}=\int \mathbf{u}_{i} \cdot \tau_{i} d t\right)$, Figure B-2. The seasonal cycle of wind work on inertial motions can be seen as a wintertime increase of the slope of $\mathrm{Pw}$ as compared to the summertime. The mean wintertime (December, January, and February) wind work is $1.1 \mathrm{mWm}^{-2}$, which is two times larger than the summertime (June, July, and August) mean of $.55 \mathrm{mWm}^{-2}$. Varying the damping coefficient for the PWP model made very little difference to the shape or magnitude of the estimated work done by the wind on inertial motions. Therefore, the intermediate value of $(1 / \mathrm{r})=5.7$ days, as suggested in Plueddemann and Farrar (2006), was chosen for the implementation of the kinetic energy model. 


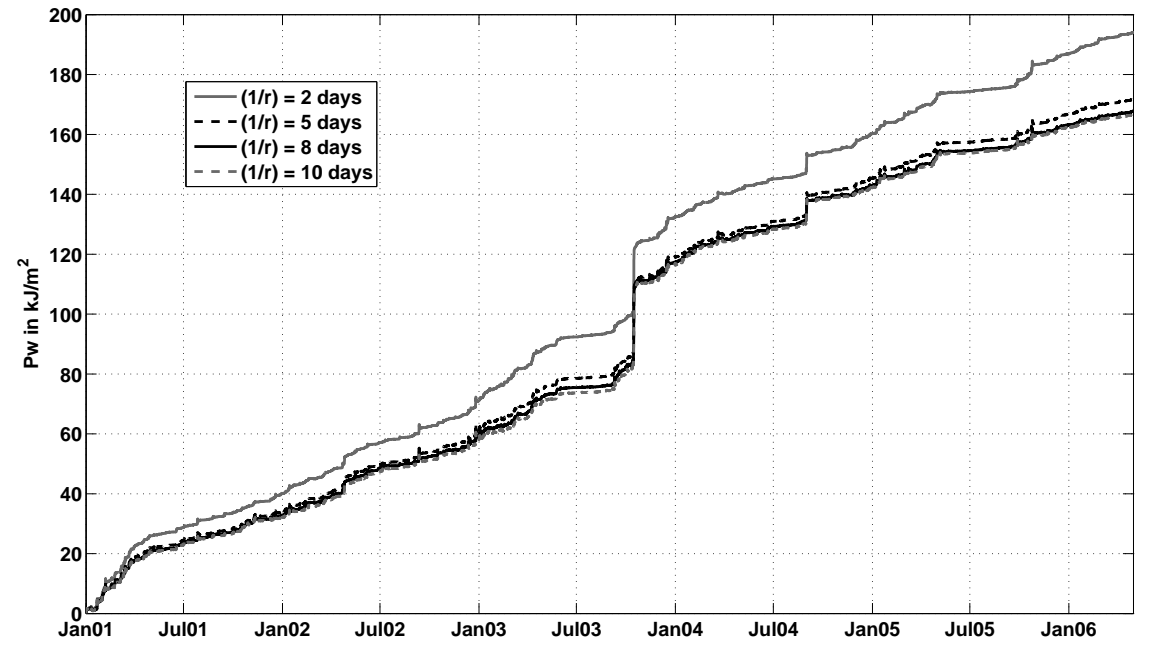

Figure B-2: Time integrated wind work for varying values of the dissipation coefficient, $r$. 


\section{Appendix C}

\section{Heat budget error calculation}

It is important to estimate the error bars on the various terms in the heat budget to demonstrate that there is a one dimensional heat budget residual which is significantly different from zero. The errors in the individual terms in the heat budget were evaluated in different ways, and therefore each is described in turn below.

The errors for the heat storage rate were estimated using the propagation of error technique (Young, 1962). Using this method, the error in a term which is dependent on many variables is determined solely from the errors in the independent variables. Therefore, the error in the heat storage rate can be determined by propagating the errors in temperature measurement and mixed layer depth determination through the heat storage rate calculation. The temperature measurement error is taken to be $0.01{ }^{\circ} \mathrm{C}$ (which is an overestimate of the manufacturer suggested $0.002{ }^{\circ} \mathrm{C}$ ). The error in mixed layer depth was estimated using a Monte Carlo simulation. For each temperature profile, random noise within the temperature measurement error was added, and the mixed layer depth determined using the criteria described above. This was done repeatedly, and then the root mean square error between mixed layer depth from the Monte Carlo simulations and the original mixed layer depth was determined. The resulting error in the estimated mixed layer depth was 10 meters. After propagating the errors in temperature and mixed layer depth through the heat content calculation, another Monte Carlo simulation was used to determine the effect 
of the two day smoothing filter on the errors. The time mean uncertainty in the heat storage rate for the EM-APEX 1636, EM-APEX 1633, and FILIS systems was found to be $\pm 140 \mathrm{Wm}^{-2}, \pm 170 \mathrm{Wm}^{-2}$, and $\pm 600 \mathrm{Wm}^{-2}$, respectively.

Because the surface heat flux term came from a model, the errors cannot be determined using the propagation of errors method. During the CLIMODE cruises, there were shipboard meteorological measurements which allow for the direct estimation of the surface heat flux. The error in the surface heat flux term is estimated here by comparison of the NAM model estimated fluxes with the shipboard fluxes. After accounting for the smoothing 2 day filter which was applied to all the heat budget terms, the error is the surface heat flux term is determined to be $\pm 150 \mathrm{Wm}^{-2}$.

Returning to the propagation of error method, errors in the depth mean advection term are dependent on the mixed layer depth error, the relative velocity error, and the temperature gradient error. The relative velocity error is dependent on the velocity measurement error (effects due to GPS position fix uncertainty were found to be negligible). Propagating the velocity measurement error through the calculations gives a relative velocity error of about $1 \mathrm{cms}^{-1}$. The error in the temperature gradient is estimated by propagating the SeaSoar temperature measurement error (again taken to be $0.01{ }^{\circ} \mathrm{C}$ ) through the temperature gradient calculation. The mean uncertainty in the depth mean advection term was found to be $\pm 120 \mathrm{Wm}^{-2}$. 


\section{Appendix D}

\section{Analytical solution behavior as the effective inertial frequency approaches the resting inertial \\ frequency}

Both components of the analytical solution of the linearized model momentum equations

$$
\begin{gathered}
u_{e}=\frac{A}{\rho_{0} h}\left(\frac{2 f_{e f f} \sin \left(f_{e f f} t\right)}{\left(f-f_{e f f}\right)\left(f+f_{e f f}\right)}-\frac{\left(f_{e f f}^{2}+f^{2}\right) \sin (f t)}{f\left(f-f_{e f f}\right)\left(f+f_{\text {eff }}\right)}\right) \\
v_{e}=\frac{2 A}{\rho_{0} h} \frac{f\left(\cos \left(f_{e f f} t\right)-\cos (f t)\right)}{\left(f-f_{e f f}\right)\left(f+f_{\text {eff }}\right)}
\end{gathered}
$$

are undefined as the effective inertial frequency approaches the resting inertial frequency. The solution at this limit can be calculated using L'Hopital's rule, which states: If $\lim _{x \rightarrow a} \frac{f(x)}{g(x)}=\frac{0}{0}$ and $\lim _{x \rightarrow a} \frac{f^{\prime}(x)}{g^{\prime}(x)}$ exists, then $\lim _{x \rightarrow a} \frac{f(x)}{g(x)}=\lim _{x \rightarrow a} \frac{f^{\prime}(x)}{g^{\prime}(x)}$. Beginning with the zonal component of flow, we first verify that the solution satisfies the condition of L'Hopital's rule:

$$
\lim _{f \rightarrow f_{e f f}} u_{e}=\frac{A}{\rho_{0} h}\left(\frac{2 f_{e f f} \sin \left(f_{e f f} t\right)-2 f_{e f f} \sin \left(f_{e f f} t\right)}{\left(f_{\text {eff }}-f_{\text {eff }}\right)\left(f_{\text {eff }}+f_{\text {eff }}\right)}\right)
$$




$$
=\frac{0}{0}
$$

Next, the derivative of the numerator and denominator of $u_{e}$ with respect to $f$ are taken as follows:

$$
\begin{gathered}
\frac{\partial}{\partial f}\left[\frac{A}{\rho_{0} h}\left(2 f f_{\text {eff }} \sin \left(f_{\text {eff }} t\right)-\left(f_{\text {eff }}^{2}+f^{2}\right) \sin (f t)\right)\right] \\
=-\frac{A}{\rho_{0} h}\left[\left(f_{\text {eff }}^{2}+f^{2}\right) t \cos (f t)-2 f \sin (f t)+2 f_{\text {eff }} \sin \left(f_{\text {eff }} t\right)\right]
\end{gathered}
$$

and

$$
\frac{\partial}{\partial f}\left[f\left(f^{2}-f_{\text {eff }}^{2}\right)\right]=3 f^{2}-f_{\text {eff }}^{2}
$$

Applying L'Hopital's rule, we see that

$$
\begin{aligned}
\lim _{f \rightarrow f_{\text {eff }}} u_{e} & =\frac{A}{\rho_{0} h} \frac{\left[-2 f_{\text {eff }}^{2} t \cos \left(f_{\text {eff }} t\right)-2 f_{\text {eff }} \sin \left(f_{\text {eff }} t\right)+2 f_{\text {eff }} \sin \left(f_{\text {eff }} t\right)\right]}{2 f_{\text {eff }}^{2}} \\
& =-\frac{A}{\rho_{0} h} t \cos \left(f_{\text {eff }} t\right)
\end{aligned}
$$

Next, we apply the same analysis to the meridional component of the velocity, beginning with verification that the analytical solution satifies the conditions for L'Hopital's rule:

$$
\begin{aligned}
\lim _{f \rightarrow f_{e f f}} v_{e} & =\frac{2 A}{\rho_{0} h}\left(\frac{f_{\text {eff }}\left(\cos \left(f_{\text {eff }} t\right)-\cos \left(f_{\text {eff }} t\right)\right)}{\left(f_{\text {eff }}-f_{\text {eff }}\right)\left(f_{\text {eff }}+f_{\text {eff }}\right)}\right) \\
& =\frac{0}{0}
\end{aligned}
$$

Derivatives of the numerator and denominator of $v_{e}$ with respect to $\mathrm{f}$ are as follows:

$$
\frac{\partial}{\partial f}\left[f\left(\cos \left(f_{\text {eff }} t\right)-\cos (f t)\right)\right]=\cos \left(f_{\text {eff }} t\right)-\cos (f t)+f t \sin (f t)
$$




$$
\frac{\partial}{\partial f}\left[f^{2}-f_{e f f}^{2}\right]=2 f
$$

Therefore,

$$
\begin{aligned}
\lim _{f \rightarrow f_{e f f}} v_{e} & =\frac{A}{\rho_{0} h} \frac{\cos \left(f_{\text {eff }} t\right)-\cos \left(f_{\text {eff }} t\right)+f_{\text {eff }} t \sin \left(f_{\text {eff }} t\right)}{2 f_{\text {eff }}} \\
& =\frac{A}{\rho_{0} h} t \sin \left(f_{\text {eff }} t\right)
\end{aligned}
$$

Looking at the limit of both solutions as the effective frequency approaches the resting inertial frequency, it can be seen that the velocity vector rotates clockwise at the inertial frequency and is linearly dependent on time, resulting in a positive input of energy from the wind into the ocean at all times. This is exactly the solution expected for a uniform ocean at rest forced with an inertially rotating wind forcing. 


\section{Bibliography}

Alford, M. H., 2001: Internal swell generation: The spatial distribution of energy flux from the wind to mixed layer near-inertial motions. J. Phys. Oceanogr., 31, $2359-2368$.

Alford, M. H., 2003: Improved global maps and 54-year history of wind-work on ocean inertial motions. Geophy. Res. Lett., 30.

Alford, M. H. and M. Whitmont, 2007: Seasonal and spatial variability of nearinertial kinetic energy from historical moored velocity records. J. Phys. Oceanogr., 37, 2022-2037.

Balmforth, N. and W. Young, 1999: Radiative damping of near-inertial oscillations in the mixed layer. J. Mar. Res., 57, 561-584.

Bradley, E., J. Godfrey, P. Coppin, and J. Butt, 1993: Observations of net heat flux into the surface mixed layer of the western equatorial pacific ocean. J. Geophys. Res., 98, 22 521-22 532 .

Cronin, M. F. and M. J. McPhaden, 1997: The upper ocean heat balance in the western equatorial pacific warm pool during september-december 1992. J. Geophys. Res., 102 (C4), 8533-8533.

Danioux, E., P. Klein, and P. Rivire, 2008: Propagation of wind energy into the deep ocean through a fully turbulent mesoscale eddy field. J. Phys. Oceanogr., 38, $2224-2241$. 
D'Asaro, E. A., 1985: The energy flux from the wind to near-inertial motions in the surface mixed layer. J. Phys. Oceanogr., 15, 1043-1059.

D’Asaro, E. A., C. Eriksen, M. Levine, P. Niiler, C. Paulson, and P. V. Meurs, 1995: Upper ocean inertial currents forced by a strong storm. part i: Data and comparisons with linear theory. J. Phys. Oceanogr., 25, 2909-2936.

Doherty, K., D. Frye, S. Liberatore, and J. Toole, 1999: A moored profiling instrument. J. Atmos. Oceanic Technol., 16, 1816-1829.

Dong, S. and K. A. Kelly, 2004: Heat budget in the gulf stream region: The importance of heat storage and advection. J. Phys. Oceanogr., 34, 1214-1231.

Fairall, C., E. Bradley, J. Hare, A. Grachev, and J. Edson, 2003: Bulk parameterization of airsea fluxes: Updates and verification for the coare algorithm. J. Climate, 16, $571-591$.

Fairall, C., E. Bradley, D. Rogers, J. Edson, and G. Young, 1996: Bulk parameterization of air-sea fluxes for tropical ocean global atmosphere coupled-ocean atmosphere response experiment. J. Geophys. Res., 101, 3747-3764.

Feng, M., P. Hacker, and R. Lukas, 1998: Upper ocean heat and salt balances in response to a westerly wind burst in the western equatorial pacific during toga coare. J. Geophys. Res., 103 (C5), 10 289-10311.

Foltz, G. R. and M. J. McPhaden, 2005: Mixed layer heat balance on intraseasonal time scales in the northwestern tropical atlantic ocean. J. Climate, 18, 4168-4184.

Garrett, C., 2001: What is the "near-inertial" band, and why is it different from the rest of the internal wave spectrum? J. Phys. Oceanogr., 31, 962-971.

Gill, A., 1984: On the behavior of internal waves in the wakes of storms. J. Phys. Oceanogr., 14, 1129-1151. 
Gonella, J., 1972: A rotary-component method for analysing meteorological and oceanographic vector time series. Deep-Sea Res., 19, 833-846.

Gregg, M., 1989: Scaling turbulent dissipation in the thermocline. J. Geophys. Res., 94, 9686-9698.

Henyey, F., J. Wright, and S. Flatte, 1986: Energy and action flow through the internal wave field: An eikonal approach. J. Geophys. Res., 91, 8487-8495.

Hoskins, B. and P. Valdes, 1990: On the existence of storm-tracks. J. Atmos. Sci., 47, 1854-1864.

Inoue, R. and M. Gregg, 2008: Mixing rates across and in the gulf stream. American Geophysical Union, Fall Meeting, December 15-19, 2008, CA, USA, Abstract: OS13B-1186.

Kelly, K. A. and B. Qui, 1995: Heat flux estimates for the western north atlantic. part ii: The upper-ocean heat balance. J. Phys. Oceanogr., 25, 2361-2373.

Kunze, E., 1985: Near-inertial wave propagation in geostrophic shear. J. Phys. Oceanogr., 15, 544-565.

Kunze, E. and T. B. Sanford, 1984: Observations of near-inertial waves in a front. J. Phys. Oceanogr., 14, 566-581.

Kunze, E., R. W. Schmitt, and J. M. Toole, 1995: The energy balance in a warm-core ring's near-inertial critical layer. J. Phys. Oceanogr., 25, 942-957.

Kwon, Y. and S. Riser, 2004a: Eighteen degree water observed using profiling floats. manuscript in preparation.

Kwon, Y.-O. and S. C. Riser, 2004b: North atlantic subtropical mode water: A history of ocean-atmosphere interaction 1961-2000. Geophy. Res. Lett., 31, L19 307, doi:10.1029/2004GL021116. 
Large, W., J. McWilliams, and P. Niiler, 1986: Upper ocean thermal response to strong autumnal forcing of the northeast pacific. J. Phys. Oceanogr., 16, 15241550.

Lavender, K. L., R. E. Davis, and W. B. Owens, 2002: Observations of open-ocean deep convection in the labrador sea from subsurface floats. J. Phys. Oceanogr., 32, $511-526$.

Leaman, K. and T. Sanford, 1975: Vertical energy propagation of inertial waves: A vector spectral analysis of velocity profiles. J. Geophys. Res., 80, 1975-1978.

Lee, D.-K. and P. P. Niiler, 1998: The inertial chimney: The near-inertial energy drainage from the ocean surface to the deep layer. J. Geophys. Res., 103 (C4), $7579-7591$.

Marshall, J., 2005: Climode: a mode water dynamics experiment in support of clivar. Variations, 3, 8-14.

McComas, C. and P. Muller, 1981: The dynamic balance of internal waves. J. Phys. Oceanogr., 11, 970-986.

McNeil, J., H. Jannasch, T. Dickey, D. McGillicuddy, M. Brzezinski, and C. Sakamoto, 1999: New chemical, bio-optical and physical observations of upper ocean response to the passage of a mesoscale eddy off bermuda. J. Geophys. Res., 104, 1553715548.

Meurs, P. V., 1998: Interactions between near-inertial mixed layer currents and the mesoscale: the importance of spatial variabilities in the vorticity field. J. Phys. Oceanogr., 28, 1363-1388.

Morrison, A., J. Billings, and K. Doherty, 2000: The mclane moored profiler: an autonomous platform for oceanographic measurements. Oceans 2000 MTS/IEEE Conference and Exhibition, 1, 353-358, iSBN:0-7803-6551-8. 
Munk, W., 1981: Internal waves and small-scale processes. Evolution of Physical Oceanography, B. Warren and C. Wunsch, Eds., The MIT Press, 264-291.

Munk, W. and C. Wunsch, 1998: Abyssal recipes ii: Energetics of tidal and wind mixing. Deep-Sea Res. I, 45, 1977-2010.

Paduan, J. D., R. A. D. Szoeke, and J. G. Richman, 1988: Balances of heat and momentum at $33.5 \mathrm{deg} \mathrm{n}, 127 \mathrm{deg} \mathrm{w}$ in the upper ocean during the mixed-layer dynamics experiment. J. Geophys. Res., 93, 8147-8160.

Park, J.-H. and D. R. Watts, 2005: Near-inertial oscillations interacting with mesoscale circulation in the southwestern japan/east sea. Geophy. Res. Lett., 32, L10 611, doi:10.1029/2005GL022936.

Pedlosky, J., 2003: Waves in the Ocean and Atmosphere. Springer.

Perkins, H., 1976: Observed effect of an eddy on near-inertial oscillations. Deep-Sea Res., 23, 1037-1042.

Pingree, R. and B. L. Cann, 1991: Drifting buoy in the field of flow of two eddies on east thulean rise (northeast atlantic). J. Geophys. Res., 96 (C9), 16 759-16777.

Plueddemann, A. and J. Farrar, 2006: Observations and models of the energy flux from the wind to mixed-layer inertial currents. Deep-Sea Res. II, 53, 5-30.

Pollard, R. and R. Millard, 1970: Comparison between observed and simulated windgenerated inertial oscillations. Deep-Sea Res., 17, 153-175.

Polzin, K., J. Toole, and R. Schmitt, 1995: Finescale parameterizations of turbulent dissipation. J. Phys. Oceanogr., 25, 306-328.

Poulain, P.-M., 1990: Near-inertial and diurnal motions in the trajectories of mixed layer drifters. J. Mar. Res., 48, 793-823.

Poulain, P.-M., D. S. Luther, and W. C. Patzert, 1992: The heat budget in the north atlantic subtropical frontal zone. J. Geophys. Res., 97 (C11), 17947-17959. 
Price, J. F., 1983: Internal wave wake of a moving storm. part i:scales, energy budget and observations. J. Phys. Oceanogr., 13, 949-965.

Price, J. F., R. A. Weller, and R. Pinkel, 1986: Diurnal cycling: Observations and models of the upper ocean diurnal heating, cooling, and wind mixing. J. Geophys. Res., 91, 8411-8427.

Richards, K., M. Inall, and N. Wells, 1995: The diurnal mixed layer and upper ocean heat budget in the western equatorial pacific. J. Geophys. Res., 100 (C4), 68656879 .

Sanford, T. B., J. H. Dunlap, J. A. Carlson, D. C. Webb, and J. B. Girton, 2005: Autonomous velocity and density profiler: Em-apex. Proceedings of the IEEE/OES Eighth Working Conference on Current Measurement Technology, 152.

Shearman, R. K., 2005: Observations of near-inertial current variability on the new england shelf. J. Geophys. Res., 110, C02 012, doi:10.1029/2004JC002341.

Skyllingstad, E. D., W. Smyth, and G. Crawford, 2000: Resonant wind-driven mixing in the ocean boundary layer. J. Phys. Oceanogr., 30, 1866-1890.

Speer, K. and E. Tziperman, 1992: Rates of water mass formation in the north atlantic ocean. J. Phys. Oceanogr., 22, 93-104.

Stevenson, J. W. and P. P. Niiler, 1983: Upper ocean heat budget during the hawaiito-tahiti shuttle experiment. J. Phys. Oceanogr., 13, 1894-1907.

Thomas, L. and R. Ferrari, 2008: Friction, frontogenesis, and the stratification of the surface mixed layer. J. Phys. Oceanogr., 38, 2501-2518.

Toole, J., K. Doherty, D. Frye, and S. Liberatore, 1999: Velocity measurements from a moored profiling instrument. Proceedings of IEEE, Sixth Working Conference on Current Measurement, March 11-13, 1999, San Diego, CA, CA, USA, 144-149. 
Worthington, L., 1959: The 18 degree water in the sargasso sea. Deep-Sea Res., 5, $297-305$.

Xue, H., J. M. B. Jr., and L. M. Goodman, 1994: Modification of the gulf stream through strong air-sea interactions in winter: Observations and numerical simulations. J. Phys. Oceanogr., 25, 533-557.

Young, H. D., 1962: Statistical treatment of experimental data. McGraw-Hill.

Young, W. and M. B. Jelloul, 1997: Propagtion of near-inertial oscillations through a geostrophic flow. J. Mar. Res., 55, 735-766. 